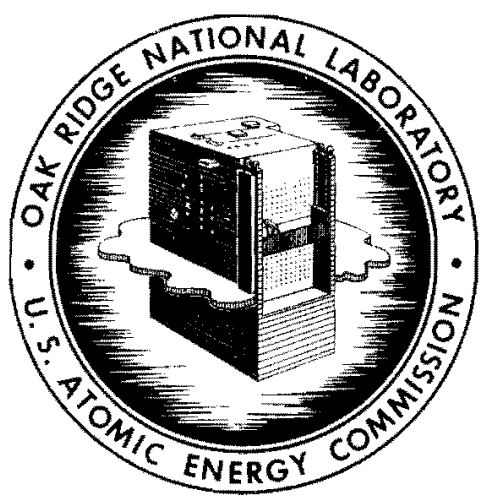

OAK RIDGE NATIONAL LABORATORY

\author{
operated by \\ UNION CARBIDE CORPORATION \\ for the
}

U.S. ATOMIC ENERGY COMMISSION

ORNL-TM-191\%)
COPYNO.

DATE - March 7, 1962

\title{
FFFECI OF REDUCED U-235 PRICE ON FUEL CYCLE COSTS
}

L. L. Bennett

ABSTRACT

A study was made to determine the effect of changes in natural uranium cost and in separative work charges on fuel cycle costs in nuclear power plants. Reactors considered were a Dresden-type boiling water reactor (BWR) and a Yankee-type pressurized water reactor (PWR), with net power ratings of 100, 300, and 500 Mwe.

Fuel cycle costs were calculated for these reactors, using either enriched uranium or U-235-thorium as the fuel material. The price schedule for uranium was based on a feed material cost of $\$ 15 / \mathrm{kg}$ uranium as $\mathrm{UF}_{6}$ and separative work costs of $\$ 30 / \mathrm{kg}$ uranium (Schedule B) and $\$ 20 / \mathrm{kg}$ uranium (Schedule C).

The present AEC price schedule for enriched uranium was also used for purposes of a reference case.

The results indicate that a reduction in present enriched uranium price to that given by schedule $B$ would reduce fuel cycle costs for the BWR plants by 0.4 to $0.5 \mathrm{mill} / \mathrm{kwh}$ for the enriched-uranium cycle, and 0.4 to $0.7 \mathrm{mill} / \mathrm{kwh}$ for the thorium cycle. Reauctions in fuel cycle costs for the PWR plants were 0.5 to 0.7 and 0.4 to $0.75 \mathrm{mill} / \mathrm{kwh}$, respectively, for the same situations.

This document contains information of a preliminary nature and was prepared primarily for internal use at the Oak Ridge National Laboratory. It is subject to revision or correction and therefore does not represent a final report. The information is not to be abstracted, reprinted or otherwise given public dis. semination without the approval of the ORNL patent branch, Legal and Information Control Department. 
- LEGAL NOTICE

This report was prepored os an account of Government sponsored work. Neither the Unitad States, nor the Commission, nor ony person acting on behalf of the Commission:

A. Mokes any warranty or representation, expressed or implied, with respect to the accuracy, completeness, or usefulness of the informotion contained in this roport, or that the use of any information, apporatus, method, or process disclosed in this report moy not infringe privotely owned rights; or

B. Assumes any liabilities with respect to the use of, or for damages resulting from the use of ony information, apparatus, methad, or process disclosed in this report.

As used in the above. "person acting on behalf of the Commission" includes any employee or contractor of the Commission, or employee of such contractor, to the extent that such employee or contractor of the Commissian, or employee of such controcter prepates, disseminates, or provides acess to, ony informotion pursuant to his employment of controct with the Commission, or his mployment with such confractor. 


\section{INTRODUCTION}

The primary factors determining the price of enriched uranium are the cost of the feed material and the cost of separative work. The present AEC established price schedule for enriched uranium is based on a natural uranium price of $\$ 23.50 / \mathrm{kg}$ uranium as $\mathrm{UF}_{6}$ and a separative work charge of $\$ 37.29 / \mathrm{kg}$ uranium. However, the price of natural uranium on the open market (for example, from Canadian sources) is lower than this at the present time, and a price of about $\$ 15 / \mathrm{kg}$ seems reasonable for the near future.

The actual cost of separative work in a gaseous diffusion plant has not been published in the open literature. However, for many years the value of $\$ 37.29 / \mathrm{kg}$ uranium has been used to calculate the AEC published price schedule for enriched uranium. It seems probable that this cost has been reduced over the years by process improvements and increased efficiency of operation. In order to determine the effect of such reductions, the separative work charges used in the reduced price schedules in this study were arbitrarily set at $\$ 30 / \mathrm{kg}$ uranium in one case and $\$ 20 / \mathrm{kg}$ in the other.

The fuel cycle costs in a Dresden-type BWR and a Yankee-type PWR were then calculated for these enriched uranium price schedules and compared with the fuel costs calculated with the present AEC price schedule.

The effect of variations in certain other cost parameters was also studied. In particular, calculations were made to determine the effect of private ownership of nuclear fuel on fuel cycle costs; this was done by considering the fuel inventory charge to be $12 \% / \mathrm{yr}$.

\section{RESULTS}

The detailed results obtained from this study are presented in the tables and graphs in Appendices $A$ and $B$. It should be noted that in all cases the data tabulated for the U-235 thorium cycle are based on a UF 6 to $\mathrm{JO}_{2}$ conversion charge of $\$ 200 / \mathrm{kg}$ uranium and a chemical reprocessing rate of $600 \mathrm{~kg} /$ day. The cost bases which were used to produce the data given by the graphs are specifically indicated in each case.

A discussion of some of the results of particular interest will be given here.

\section{Reduction in Fuel Cycle Cost}

The primary objective of this study was to determine the reduction in fuel cycle cost which would be obtained by lowering the price of enriched uranium. These results are presented in Tables 1 and 2 . Fuel cost reductions obtained in going to schedule $B$ were in the range 0.4 to $0.7 \mathrm{mill} / \mathrm{kwh}$ and for Schedule $\mathrm{C}$ were 0.7 to $1.2 \mathrm{mill} / \mathrm{kwh}$. 
TABLE 1. FUEL COST REDUCTION FOR BOILING WATER REACTORS

\begin{tabular}{|c|c|c|c|c|c|c|}
\hline \multirow{3}{*}{$\begin{array}{l}\text { Reactor } \\
\text { Output, } \\
\text { Mwe }\end{array}$} & & \multicolumn{2}{|c|}{ Enriched Uranium Fuel } & \multicolumn{2}{|c|}{ U-235 Thorium Fuel } \\
\hline & Lease & & Minimum & Fuel Cost & Minimum & Fuel Cost \\
\hline & $\begin{array}{c}\text { Charge, } \\
\%\end{array}$ & $\begin{array}{l}\text { Price } \\
\text { Schedule }\end{array}$ & $\begin{array}{l}\text { Fuel Cost } \\
\text { mills/kwh }\end{array}$ & $\begin{array}{l}\text { Reduction } \\
\text { mills/kwh }\end{array}$ & $\begin{array}{l}\text { Fuel Cost } \\
\text { mills/kwh }\end{array}$ & $\begin{array}{l}\text { Reduction } \\
\text { mills/kwh }\end{array}$ \\
\hline \multirow[t]{3}{*}{100} & $4-3 / 4$ & A & 2.912 & $(\operatorname{Re} f)$ & 2.959 & $(\operatorname{Ref})$ \\
\hline & & B & 2.456 & 0.456 & 2.546 & 0.413 \\
\hline & & $\mathrm{C}$ & 2.189 & 0.723 & 2.218 & 0.741 \\
\hline \multirow[t]{3}{*}{300} & $4-3 / 4$ & A & 2.822 & $(\operatorname{Ref})$ & 2.838 & $(\operatorname{Ref})$ \\
\hline & & B & 2.388 & 0.434 & 2.452 & 0.386 \\
\hline & & C & 2.135 & 0.687 & 2.147 & 0.691 \\
\hline \multirow[t]{3}{*}{500} & $4-3 / 4$ & A & 2.808 & $(\operatorname{Ref})$ & 2.823 & $(\operatorname{Ref})$ \\
\hline & & B & 2.376 & 0.432 & 2.435 & 0.388 \\
\hline & & $\mathrm{C}$ & 2.125 & 0.683 & 2.136 & 0.687 \\
\hline \multirow[t]{3}{*}{100} & 12 & A & 3.442 & $(\operatorname{Ref})$ & 4.195 & $(\operatorname{Re} f)$ \\
\hline & & B & 2.898 & 0.544 & 3.488 & 0.707 \\
\hline & & $\mathrm{C}$ & 2.599 & 0.843 & 2.974 & 1.221 \\
\hline \multirow[t]{3}{*}{300} & 12 & A & 3.325 & $(\operatorname{Re} f)$ & 4.041 & $(\operatorname{Ref})$ \\
\hline & & B & 2.810 & 0.515 & 3.367 & 0.674 \\
\hline & & $\mathrm{C}$ & 2.522 & 0.803 & 2.874 & 1.167 \\
\hline \multirow[t]{3}{*}{500} & 12 & A & $3 \cdot 302$ & $(\operatorname{Ref})$ & 4.029 & $(\operatorname{Ref})$ \\
\hline & & $B$ & 2.792 & 0.510 & 3.359 & 0.670 \\
\hline & & $\mathrm{C}$ & 2.508 & 0.794 & 2.869 & 1.160 \\
\hline
\end{tabular}


TABIE 2. FUEL COST REDUCTIONS FOR PRESSURIZED WATER REACTORS

\begin{tabular}{|c|c|c|c|c|c|c|}
\hline \multirow{3}{*}{$\begin{array}{l}\text { Reactor } \\
\text { Output } \\
\text { Mwe }\end{array}$} & \multirow{2}{*}{$\begin{array}{l}\text { Fuel } \\
\text { Lease }\end{array}$} & & \multicolumn{2}{|c|}{ Enriched Uranium Fuel. } & \multicolumn{2}{|c|}{ U-235 Thorium Fuel } \\
\hline & & & Minimum & Fuel Cost & Minimum & Fuel Cost \\
\hline & $\begin{array}{c}\text { Charge, } \\
\%\end{array}$ & $\begin{array}{l}\text { Price } \\
\text { Schedule }\end{array}$ & $\begin{array}{l}\text { Fuel Cost } \\
\text { mills/kwh }\end{array}$ & $\begin{array}{l}\text { Reduction } \\
\text { mills/kwh }\end{array}$ & $\begin{array}{l}\text { Fuel Cost } \\
\mathrm{mills} / \mathrm{kwh}\end{array}$ & $\begin{array}{l}\text { Reduction } \\
\text { mills/kwh }\end{array}$ \\
\hline \multirow[t]{3}{*}{100} & $4-3 / 4$ & A & 2.835 & $(\operatorname{Ref})$ & 2.684 & $(\operatorname{Re} f)$ \\
\hline & & B & 2.337 & 0.498 & 2.244 & 0.44 \\
\hline & & $\mathrm{C}$ & 2.038 & 0.797 & 1.904 & 0.780 \\
\hline \multirow[t]{3}{*}{300} & $4-3 / 4$ & A & 2.654 & $(\operatorname{Re} f)$ & 2.495 & $(\operatorname{Ref})$ \\
\hline & & B & 2.185 & 0.469 & 2.098 & 0.397 \\
\hline & & $\mathrm{C}$ & 1.91 .4 & 0.740 & 1.789 & 0.706 \\
\hline \multirow[t]{3}{*}{500} & $4-3 / 4$ & A & 2.610 & $(\operatorname{Ref})$ & 2.451 & $(\operatorname{Re} f)$ \\
\hline & & B & 2.149 & 0.461 & 2.062 & 0.389 \\
\hline & & $\mathrm{C}$ & 1.883 & 0.727 & 1.761 & 0.690 \\
\hline \multirow[t]{3}{*}{100} & 12 & A & 3.571 & $(R \in f)$ & 3.972 & $(\operatorname{Ref})$ \\
\hline & & B & 2.911 & 0.660 & 3.227 & 0.745 \\
\hline & & $\mathrm{C}$ & 2.538 & 1.033 & 2.688 & 1.284 \\
\hline \multirow[t]{3}{*}{300} & 12 & A & 3.311 & $(\operatorname{Re} f)$ & 3.688 & (Ref) \\
\hline & & B & 2.706 & 0.605 & 3.016 & 0.672 \\
\hline & & $\mathrm{C}$ & 2.362 & 0.949 & 2.513 & 1.175 \\
\hline \multirow[t]{3}{*}{500} & 12 & A & 3.249 & $(\operatorname{Re} f)$ & 3.642 & $(\operatorname{Re} I)$ \\
\hline & & B & 2.654 & 0.595 & 2.972 & 0.670 \\
\hline & & $\mathrm{C}$ & 2.323 & 0.926 & 2.483 & 1.159 \\
\hline
\end{tabular}




\section{Effect of Private Ownership of Fuel}

If the AEC should adopt a policy of private ownership of special nuclear materials, then the money spent for fuel would normally be considered to have an interest charge of 12 to $13 \%$ per year rather than the present $A E C$ use charge of $4-3 / 4 \%$ per year. This higher fuel interest charge increased fuel cost by about 0.5 to $0.7 \mathrm{mill} / \mathrm{kwh}$ on the enriched uranium cycle and by 0.7 to $1.3 \mathrm{mill} / \mathrm{kwh}$ on the U-235 thorium cycle. A comparison of fuel costs on Schedule A at 4-3/4\% with those on Schedules $\mathrm{B}$ and $\mathrm{C}$ at $12 \%$ indicates that a change to private ownership, with a corresponding reduction in enriched uranium price, would reduce fuel cycle costs for the enriched uranium cycle. On the U-235 thorium cycle, however, the higher interest rate is not completely compensated by the reduction in U-235 price, and the fuel costs are higher with private ownership in all cases.

\section{Comparison of Enriched Uranium and U-235 Thorium Cycles}

With 4-3/4\% fuel lease charge, the fuel cost for the enriched uranium cycle and the U-235 thorium cycle are not greatly different. The BWR has slightly lower fuel costs on the enriched uranium cycle, while for the PWR the thorium cycles have fuel costs about 0.1 to $0.2 \mathrm{mill} / \mathrm{kwh}$ lower than the enriched uranium cycles. In the PWR, use of thorium as a fertile material is better than $\mathrm{U}-238$ because thorium has a larger thermal absorption cross section and can compete for neutrons more favorably relative to absorptions by the stainless steel cladding. In the BWR, the cladding is zircaloy, which absorbs very few neutrons in any case; thus, the larger cross section of thorium is not so important.

When the interest charge on fuel is increased to $12 \%$, the high value of the highly enriched uranium used in the thorium cycle results in a high inventory charge. Thus, the fuel costs with the thorium cycle are considerably higher than with enriched uranium for both the PWR and the BWR, with respect to the first cycle.

4. Comparison of Fuel Costs for BWR and PWR

The BWR and PWR have about the same fuel costs on the enriched uranium cycle, although the PWR costs are slightly lower. With U-235 thorium as the fuel, however, the PWR shows a definite cost advantage; here, fuel costs for the PWR are about $0.3 \mathrm{mill} / \mathrm{kwh}$ lower than the BWR.

\section{Effect of Changes in Conversion Costs}

The cost of converting fully enriched uranium from $\mathrm{UF}_{6}$ to $\mathrm{UO}_{2}$ is more uncertain than most of the items contributing to fuel cycle costs. In this study, the conversion cost was assumed to be $\$ 200 / \mathrm{kg}$ uranium in all the results which were tabulated. A number of the cycles were recalculated using a conversion cost of $\$ 400 / \mathrm{kg}$, and some of these are 
presented in the graphs. Since a relatively small amount of uranium is being converted, this change in conversion cost increases fuel costs by only about $0.05 \mathrm{mfl} / \mathrm{kwh}$.

6. Effect of Changing Chemical Reprocessing Plant Throughput Rate

Most of the fuel cost calculations made during the ORNI study of U-233 and plutonium fuel values were based on the chemical reprocessing rates given in WASH-743. A number of the U-235 thorium cases in this study were also calculated on this basis for comparison with results obtained with the rates given in the AEC cost handbook. The rates used are listed in Table 3. Fuel costs for the BWR were in the range of 0.04 to $0.08 \mathrm{mill} / \mathrm{kwh}$ lower with the WASH -743 rates. Optimum enrichments for the BWR cycles were near 2.5 to $3 \% \mathrm{U}-235$, and the WASH-743 rates were higher than $600 \mathrm{~kg} /$ day in this range. Fuel costs for the PWR were slightly lower ( $\sim 0.01$ mill) with the $600 \mathrm{~kg} /$ day processing rate. Optimum enrichments were in the range of 4 to $4.5 \% \mathrm{U}-235$, and the WASH -743 rates were less than $600 \mathrm{~kg} /$ day in this range.

TABLE 3. CHEMICAI REPROCESSING RATES FOR THORIUM

Enrichment, $\%$ Fissile in Th
Chemical Processing Rate, $\mathrm{kg} /$ day WASH $-743^{*}$

$\begin{array}{rr}1000 & 600 \\ 950 & 600 \\ 700 & 600 \\ 550 & 600 \\ 460 & 600 \\ 390 & 600\end{array}$

*The rates published in WASH- 743 have been reduced to allow for the increased reactivity of the $\mathrm{U}-233$ which is bred in.

\section{DETAILS OF CALCULATION}

The major reactor characteristics used in this study are presented in Table 4. These parameters were obtained by taking the basic data from the Dresden and Yankee reference designs and varying the core sizes to obtain the desired power level.

The reactivity lifetime at various enrichments was calculated for each reactor using the Expire ${ }^{1}$ code. This calculation assumes that the 
TABLE 4. SUMMARY OF REACTOR CHARACTERISTICS

\begin{tabular}{|c|c|c|c|c|c|}
\hline Dre & $\mathrm{n}-\mathrm{Ty}$ & BWR & & $e-T y$ & PWR \\
\hline 100 & 300 & 500 & 100 & 300 & 500 \\
\hline Mwe & Mwe & Mwe & Mwe & Mwe & Mwe \\
\hline
\end{tabular}

Heat Balance

Total Reactor Power, Mwt

Net Plant Power, Mwe

Net Plant eff, of

$\begin{array}{llllll}348 & 1043 & 1738 & 356 & 1069 & 1782\end{array}$

$\begin{array}{llllll}100 & 300 & 500 & 100 & 300 & 500\end{array}$

$\begin{array}{llllll}28.75 & 28.75 & 28.75 & 28.06 & 28.06 & 28.06\end{array}$

$\underline{\text { Reactor Core }}$

Active Height, ft.

$\begin{array}{llllll}7.12 & 10.28 & 12.18 & 7.27 & 10.48 & 12.42\end{array}$

Active Radius, ft.

$\begin{array}{lll}4.42 & 6.37 \quad 7.56\end{array}$

2.99

4.31

5.11

Core Loading, metric

tons uranium

Specific Power, kwt $/ \mathrm{kgU}$

28.98

86.94

$144.9 \quad 18.21$

$54.63 \quad 91.04$

Power Density, kwt/liter

12.00

19.57

28.10

61.80

Fuel Elements

Fuel Material

Clad Material

Pellet Diameter, in.

Clad Thickness, in.

$\mathrm{UO}_{2}$ Pellets

$\mathrm{z} r-2$

.493

.030

$\mathrm{UO}_{2}$ Pellets

304 SS

.290

.021

Fuel Assemblies

Total Number

Elements per Assembly

330

686

36

953

72

148

208

Coolant

$\mathrm{H}_{2} \mathrm{O}$

$\mathrm{H}_{2} \mathrm{O}$

reactor operates on a batch cycle in which all fuel in the reactor is exposed to the same neutron flux at a given time, and the flux changes with time to maintain a fixed power level. This is commonly called a "uniform batch" cycle.

Using the lifetime and composition results obtained from Expire, an economic analysis of the cycles was made using a modified Epitaph ${ }^{2}$ fuel-cost calculation. The following features are inherent to this calculation: 
1. The use charge on nuclear materials is based on the time value of the material as follows:

a) initial value of fuel taken for the preirradiation time;

b) average value of fuel taken during irradiation (not including plutonium value); and

c) value of spent fuel taken for post-irradiation time (including plutonium).

2. Interest charges on fabrication capital are based on a linear write-off of the fabrication cost over the irradiation time.

The following equations were used to compute the price of enriched uranium in which the $\mathrm{U}-233$ may be present:

a) $f_{23}=$ weight fraction $U-233$ in total uranium,

b) $f_{25}=$ weight fraction $\mathrm{J}-235$ in total uranium,

c) $\mathrm{E}=\mathrm{f}_{23}+\mathrm{f}_{25}$, fissile enrichment,

a) $C_{p}(E)=C_{\text {sep }}\left[(2 E-1) \log \frac{\left(1-X_{O}\right) E}{X_{O}(1-E)}+\frac{\left(I-2 X_{O}\right)\left(E-X_{O}\right)}{X_{O}\left(1-X_{O}\right)}\right]$.

The value of mixtures containing U-233, U-235 were taken as follows:

e) If $f_{23}=0$, i.e., U-235, U-238 mixture

$$
V_{u}=C_{p}(E) \text {, price of cascade uranium. }
$$

f) If f23 $\neq 0$, i.e., contains U-233

$$
v_{u}=\left[f_{25} \cdot v^{25}+f_{23} \cdot v^{23}\right] \cdot \frac{C_{p}(E)}{E \cdot v^{25}} \text {, price }
$$

taken for uranium containing $\mathrm{v}-233$.

The economics parameters used to calculate the fuel cost are presented in Tables 3,5 , and 6 .

The first three schedules listed in Table 5 are of primary interest since the value of bred materials has been kept in proportion to the U- 235 
value in these. Schedules $D$ and $E$ were included only to show the effect of a reduction in $\mathrm{U}-235$ price while maintaining the U-233 and plutonium prices at high levels. Therefore, in most cases, results will be presented only for schedules $A, B$, and $C$, with results from Schedules D and $E$ included in only a few cases.

TABLE 5. COST PARAMETERS USED IN PRICE SCHEDULES

\begin{tabular}{|c|c|c|c|c|c|c|}
\hline $\begin{array}{l}\text { Cost } \\
\text { Sched- } \\
\text { ule }\end{array}$ & $\begin{array}{l}\text { Feed } \\
\text { Mat' } 1 . \\
\text { Cost, } \\
\$ / \mathrm{kg} \mathrm{U}\end{array}$ & $\begin{array}{l}\text { Sep. } \\
\text { Work } \\
\text { Cost } \\
\$ / \mathrm{kg} \mathrm{U}\end{array}$ & $\begin{array}{l}\text { Tails } \\
\text { Comp. } \\
w / f \\
U-235\end{array}$ & $\begin{array}{c}\mathrm{U}-235 \text { value } \\
\text { at } 95 \% \\
\text { Enrich. } \\
\$ / \mathrm{gm} \mathrm{U}-235\end{array}$ & $\begin{array}{c}\text { Pu Price } \\
\text { as Nitrate } \\
\$ / \mathrm{gm}\end{array}$ & $\begin{array}{l}\text { U-233 Price } \\
\text { as Nitrate } \\
\$ / \mathrm{gm}\end{array}$ \\
\hline A & 23.50 & 37.29 & 0.00277 & 13.70 & 8.00 & 15.00 \\
\hline B & 15.00 & 30.00 & 0.0030 & 10.25 & 6.00 & 11.20 \\
\hline $\mathrm{C}$ & 15.00 & 20.00 & 0.0026 & 7.82 & 4.50 & 8.60 \\
\hline D & 15.00 & 30.00 & 0.0030 & 10.25 & 8.00 & 15.00 \\
\hline $\mathrm{E}$ & 15.00 & 20.00 & 0.0026 & 7.82 & 8.00 & 15.00 \\
\hline
\end{tabular}


TABIE 6. ECONOMICS PARAMETERS

\begin{tabular}{|c|c|}
\hline $\begin{array}{l}\text { Preirradiation conversion loss of uranium, of } \\
\text { Ioss during fabrication. of }\end{array}$ & $\begin{array}{l}1.0 \\
1.0\end{array}$ \\
\hline Cost of converting low enrichment $\mathrm{UF}_{6}$ to $\mathrm{UO}_{2}$ & $\begin{array}{l}\text { From Figure } 450-1 \text { of Vol, } \\
4 \text { of Nuclear Power Plant } \\
\text { Cost Evaluation Handbook }\end{array}$ \\
\hline Cost of converting fully enriched $\mathrm{UF}_{6}$ to $\mathrm{UO}_{2}$ & $\$ 200$ or $\$ 400 / \mathrm{kg} U$ \\
\hline Preirradiation shipping cost, $\$ / \mathrm{kg}$ & 3.0 \\
\hline Post-irradiation shipping cost, $\$ / \mathrm{kg}$ & 16.0 \\
\hline Chemical reprocessing charge, \$/day & 17,000 \\
\hline Post-irradiation losses of uranium* and plutonium, $\%$ & $1.3 \%$ and $1.0 \%$, respectively \\
\hline 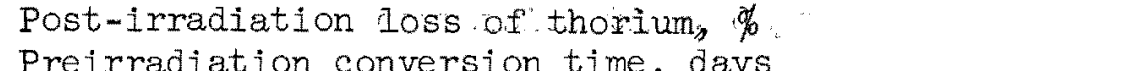 & 2.0 \\
\hline Preirradiation conversion time, days & 30 \\
\hline Core fabrication time, days & 135 \\
\hline Preirradiation shipping time, days & 45 \\
\hline Post-irradiation cooling time, days & 120 \\
\hline Shipping time to chemical reprocessing plant, days & 30 \\
\hline Post-irradiation conversion time, days & 30 \\
\hline Post-irradiation conversion cost of uranium*, $\$ / \mathrm{kg}$ & 5.6 \\
\hline Post-irradiation conversion cost of thorium, $\$ / \mathrm{kg}$ & 2.2 \\
\hline Interest rate on purchased thorium, $\% / y r$ & 12 \\
\hline Interest rate on borrowed money, $\% / y r$ & 12 \\
\hline Interest rate on uranium and plutonium, $\% / y r$ & $4-3 / 4$ or 12.0 \\
\hline Value of $\mathrm{U}-235, \$ / \mathrm{kg}$ of uranium & $\begin{array}{l}\text { Based on ideal diffusion } \\
\text { plant cost equation with } \\
\text { parameters listed in } \\
\text { Table } 5\end{array}$ \\
\hline Value of bred materials & See Table 5 \\
\hline Value of thorium, $\$ / \mathrm{kg}$ & 22 \\
\hline Load factor & 0.8 \\
\hline $\begin{array}{l}\text { Fabrication cost of fuel, not including con- } \\
\text { version or preirradiation inventory charges }\end{array}$ & \\
\hline or preirradiation losses, $\$ / \mathrm{kg}$ of uranium & Reactor \\
\hline & BWR \\
\hline & 95.0 \\
\hline
\end{tabular}

*Highly enriched uranium containing U-233 is sold as the nitrate; losses are $1 \%$ and no conversion cost. 


\section{REFERENCES}

1. Jaye, S., "Expire - A Reactivity Lifetime Calculation," ORNL-CF60-10-113, october 13, 1960.

2. Bennett, I. I., "Epitaph - A Reactor Fuel Cost Calculation," ORNLCF-61-3-105, March 21, 1961.

3. USAEC, Nuclear Power Plants Cost Evaluation Handbook, Volume 4, "Fuel Cycle Costs," December 31, 1961.

4. "AEC Reference Fuel Processing Plant," WASH-743, October 1957. 
$+$ 
$-15-$

APPENDIX A

DETAIIED RESULTS FOR BOILTNG WATER

REACTOR CASES 
TABLE A-1

FUEL CYCLE COST FOR BOILING WATER REACTORS

FUELED WITH ENRICHED URANIUM

Use Charge on Uranium and Plutonium: $4-3 / 4 \%$

\begin{tabular}{|c|c|c|c|c|c|c|c|c|c|}
\hline \multirow[b]{2}{*}{$\begin{array}{l}\text { Cost } \\
\text { sched- } \\
\text { ule }\end{array}$} & \multirow[b]{2}{*}{$\begin{array}{l}\text { Power } \\
\text { Level, } \\
\text { Mwe }\end{array}$} & \multicolumn{8}{|c|}{ Cost Breakdown, mills/kwhr(e) } \\
\hline & & $\begin{array}{l}\text { Fabri- } \\
\text { cation }\end{array}$ & Shipping & Burmup & $\begin{array}{l}\text { Chem. } \\
\text { Proc. }\end{array}$ & $\begin{array}{l}\text { Inven- } \\
\text { tory }\end{array}$ & Interest & $\begin{array}{c}\text { Pu } \\
\text { Credit }\end{array}$ & $\begin{array}{l}\text { Net } \\
\text { Fuel } \\
\text { Cost } \\
\end{array}$ \\
\hline \multirow[t]{3}{*}{ A } & 100 & .714 & .125 & 1.468 & .163 & .362 & .395 & .315 & 2.912 \\
\hline & 300 & .686 & .120 & 1.435 & .138 & $\cdot 358$ & .393 & $\cdot 308$ & 2.822 \\
\hline & 500 & .680 & .119 & 1.428 & .134 & .360 & .393 & .306 & 2.808 \\
\hline \multirow[t]{3}{*}{ B } & 100 & .610 & .106 & 1.115 & .140 & .311 & .389 & .215 & 2.456 \\
\hline & 300 & .588 & .102 & 1.092 & .120 & .308 & .388 & .210 & 2.388 \\
\hline & 500 & .584 & .102 & 1.087 & .116 & .309 & .387 & .209 & 2.376 \\
\hline \multirow[t]{3}{*}{$\mathrm{C}$} & 100 & .537 & .093 & .906 & .124 & .291 & .387 & .149 & 2.189 \\
\hline & 300 & .519 & .090 & .890 & .107 & .289 & .386 & .146 & 2.135 \\
\hline & 500 & .516 & .089 & .886 & .104 & .290 & .385 & .145 & 2.125 \\
\hline \multirow[t]{3}{*}{ D } & 100 & .610 & .106 & 1.115 & .141 & .312 & .389 & .286 & 2.387 \\
\hline & 300 & .588 & .102 & 1.092 & .121 & .310 & .388 & .280 & 2.321 \\
\hline & 500 & .584 & .102 & 1.087 & .117 & .312 & .387 & .279 & 2.310 \\
\hline \multirow[t]{3}{*}{$E$} & 100 & .607 & .106 & .879 & .141 & .254 & .384 & .286 & 2.085 \\
\hline & 300 & .586 & .102 & .862 & .121 & .253 & .383 & .280 & 2.027 \\
\hline & 500 & .516 & .089 & .886 & .105 & .294 & .385 & .258 & 2.017 \\
\hline
\end{tabular}




\section{TABLi巴 A-2}

FUEL CYCLE COST FOR BOILING WATER REACTORS

FUELED WITH ENRICHED URANTUM

Use Charge on Uranium and Plutonium: $12 \%$

\begin{tabular}{|c|c|c|c|c|c|c|c|c|c|}
\hline \multirow[b]{2}{*}{$\begin{array}{l}\text { Cost } \\
\text { Sched- } \\
\text { ule }\end{array}$} & \multirow[b]{2}{*}{$\begin{array}{l}\text { Power } \\
\text { Level } \\
\text { Mwe } \\
\end{array}$} & \multicolumn{8}{|c|}{ Cost Breakdown, mills/kwhr(e) } \\
\hline & & $\begin{array}{l}\text { Fabri- } \\
\text { cation }\end{array}$ & Shipping & Burnup & $\begin{array}{l}\text { Chem. } \\
\text { Proc. }\end{array}$ & $\begin{array}{l}\text { Inven- } \\
\text { tory }\end{array}$ & Interest & $\begin{array}{c}\mathrm{Pu} \\
\text { Credit }\end{array}$ & $\begin{array}{l}\text { Net } \\
\text { Fuel } \\
\text { Cost }\end{array}$ \\
\hline \multirow[t]{3}{*}{ A } & 100 & .869 & .155 & 1.408 & .200 & .747 & .418 & .355 & 3.442 \\
\hline & 300 & .829 & .148 & 1.372 & .169 & .738 & .416 & .347 & 3.325 \\
\hline & 500 & .920 & .165 & 1.326 & .184 & .663 & .414 & .370 & 3.302 \\
\hline \multirow[t]{3}{*}{ B } & 100 & .710 & .125 & 1.073 & .161 & .657 & .408 & .236 & 2.898 \\
\hline & 300 & .825 & .148 & .998 & .168 & .528 & .403 & .260 & 2.810 \\
\hline & 500 & .816 & .146 & .992 & .162 & .532 & .403 & .259 & 2.792 \\
\hline \multirow[t]{3}{*}{$\mathrm{C}$} & 100 & .707 & .125 & .849 & .160 & .535 & .400 & .177 & 2.599 \\
\hline & 300 & .680 & .120 & .831 & .136 & .529 & .399 & .173 & 2.522 \\
\hline & 500 & .673 & .119 & .826 & .132 & .532 & .398 & .172 & 2.508 \\
\hline \multirow[t]{3}{*}{$D$} & 100 & .710 & .125 & 1.073 & .162 & .662 & .408 & .315 & 2.825 \\
\hline & 300 & .825 & .148 & .998 & .169 & .536 & .403 & .347 & 2.732 \\
\hline & 500 & .816 & .146 & .992 & .163 & .541 & .403 & .345 & 2.716 \\
\hline \multirow[t]{3}{*}{$E$} & 100 & .707 & .125 & .849 & .162 & .544 & .400 & .315 & 2.472 \\
\hline & 300 & .680 & .120 & .831 & .138 & .540 & .399 & .308 & 2.400 \\
\hline & 500 & .673 & .119 & .826 & .133 & .545 & .398 & .306 & 2.388 \\
\hline
\end{tabular}


TABLE A-3

FUEL CYCLE COST FOR BOILTNG WATER REACTORS

FUELED WITH U-235 IN THORIUM

Use Charge on Uranium: $4-3 / 4 \%$

\begin{tabular}{|c|c|c|c|c|c|c|c|c|}
\hline \multirow[b]{2}{*}{$\begin{array}{l}\text { Cost } \\
\text { Sched- } \\
\text { ule }\end{array}$} & \multirow[b]{2}{*}{$\begin{array}{l}\text { Power } \\
\text { Level, } \\
\text { Mwe }\end{array}$} & \multicolumn{7}{|c|}{ Cost Breakdown, mills $/ \mathrm{kwhr}(\mathrm{e})$} \\
\hline & & $\begin{array}{l}\text { Fabri- } \\
\text { cation }\end{array}$ & Shipping & $\begin{array}{l}\text { Total } \\
\text { Burnup } \\
\text { Cost }\end{array}$ & $\begin{array}{l}\text { Chem. } \\
\text { Proc. }\end{array}$ & $\begin{array}{l}\text { Inven- } \\
\text { tory }\end{array}$ & Interest & $\begin{array}{l}\text { Net } \\
\text { Fuel } \\
\text { Cost } \\
\end{array}$ \\
\hline \multirow[t]{3}{*}{ A } & 100 & .620 & .116 & .731 & .206 & .903 & .383 & 2.959 \\
\hline & 300 & .569 & .106 & .705 & .173 & .905 & .380 & 2.838 \\
\hline & 500 & .556 & .104 & .698 & .166 & .920 & .379 & 2.823 \\
\hline \multirow[t]{3}{*}{ B } & 100 & .509 & .095 & .652 & .165 & .751 & .374 & 2.546 \\
\hline & 300 & .564 & .106 & .536 & .170 & .706 & .370 & 2.452 \\
\hline & 500 & .551 & .104 & .530 & .163 & .718 & .369 & 2.435 \\
\hline \multirow[t]{3}{*}{$\mathrm{C}$} & 100 & .469 & .088 & .522 & .151 & .621 & .367 & 2.218 \\
\hline & 300 & .473 & .089 & .476 & .140 & .605 & .364 & 2.147 \\
\hline & 500 & .465 & .087 & .472 & .135 & .613 & .364 & 2.136 \\
\hline \multirow[t]{3}{*}{ D } & 100 & .554 & .104 & .387 & .183 & $\cdot 777$ & $\cdot 373$ & 2.378 \\
\hline & 300 & .564 & .106 & .310 & .172 & .761 & $\cdot 370$ & 2.283 \\
\hline & 500 & .551 & .104 & .308 & .165 & $\cdot 775$ & .369 & 2.272 \\
\hline \multirow[t]{3}{*}{$E$} & 100 & .550 & .104 & .082 & .182 & .671 & .365 & 1.954 \\
\hline & 300 & .560 & .106 & .017 & .171 & .662 & .363 & 1.879 \\
\hline & 500 & .548 & .104 & .019 & .164 & .677 & .362 & 1.874 \\
\hline
\end{tabular}


TABLE A-4

FUEL CYCIE COST FOR BOILING WATER REACTORS

FUELED WITH U-235 IN THORIUM

Use Charge on Uranium: 12\%

\begin{tabular}{|c|c|c|c|c|c|c|c|c|}
\hline \multirow[b]{2}{*}{$\begin{array}{l}\text { Cost } \\
\text { Sched- } \\
\text { ule }\end{array}$} & \multirow[b]{2}{*}{$\begin{array}{l}\text { Power } \\
\text { Level, } \\
\text { Mwe }\end{array}$} & \multicolumn{7}{|c|}{ Cost Breakdown, mills/kwhr(e) } \\
\hline & & $\begin{array}{l}\text { Fabri- } \\
\text { cation }\end{array}$ & Shipping & $\begin{array}{l}\text { TotaI } \\
\text { Burnup } \\
\text { Cost }\end{array}$ & $\begin{array}{l}\text { Chem. } \\
\text { Proc. }\end{array}$ & $\begin{array}{l}\text { Inven- } \\
\text { tory }\end{array}$ & Interest & $\begin{array}{l}\text { Net } \\
\text { Fue } 1 \\
\text { Cost }\end{array}$ \\
\hline \multirow[t]{3}{*}{ A } & 100 & .620 & .116 & .731 & .206 & 2.096 & .426 & 4.195 \\
\hline & 300 & .745 & .141 & .540 & .230 & 1.968 & .417 & 4.041 \\
\hline & 500 & .719 & .136 & .533 & .218 & 2.007 & .416 & 4.029 \\
\hline \multirow[t]{3}{*}{$\mathrm{B}$} & 100 & .615 & .1 .16 & .556 & .202 & 1.594 & .405 & 3.488 \\
\hline & 300 & .633 & .120 & .479 & .192 & 1.543 & .400 & 3.367 \\
\hline & 500 & .616 & .1 .17 & .474 & .183 & 1.570 & .399 & 3.359 \\
\hline \multirow[t]{3}{*}{$\mathrm{C}$} & 100 & .550 & .104 & .454 & .179 & 1.295 & .392 & 2.974 \\
\hline & 300 & .560 & .106 & .401 & .167 & 1.253 & .387 & 2.874 \\
\hline & 500 & .548 & .104 & .397 & .161 & 1.273 & .386 & 2.869 \\
\hline \multirow[t]{3}{*}{$D$} & 100 & .615 & .116 & .318 & .204 & 1.722 & .405 & 3.380 \\
\hline & 300 & .633 & .120 & .231 & .194 & 1.680 & .400 & 3.258 \\
\hline & 500 & .616 & .117 & .230 & .186 & 1.715 & .399 & 3.263 \\
\hline \multirow[t]{3}{*}{$E$} & 100 & .698 & .133 & -.076 & .234 & 1.391 & .390 & 2.770 \\
\hline & 300 & .735 & .141 & -.166 & .227 & 1.345 & .387 & 2.669 \\
\hline & 500 & .710 & .136 & -.161 & .215 & 1.385 & .386 & 2.671 \\
\hline
\end{tabular}




\section{TABLE A-5}

COST PARAMETERS OF OPTIMUM CASES FOR BOILING

WATER REACTORS FUELED WITH ENRICHED URANIUM

Use Charge on Uranium and Plutonium: $4-3 / 4 \%$

\begin{tabular}{|c|c|c|c|c|c|c|c|}
\hline $\begin{array}{l}\text { Cost } \\
\text { Sched- } \\
\text { ule } \\
\end{array}$ & $\begin{array}{l}\text { Power } \\
\text { Level, } \\
\text { Mwe }\end{array}$ & $\begin{array}{l}\text { Initial } \\
\text { wt. of } \\
U-235\end{array}$ & $\begin{array}{l}\text { Final } \\
\text { wt. \% } \\
\text { U-235 }\end{array}$ & $\begin{array}{l}\text { Final } \mathrm{Pu} \\
\mathrm{gm} / \mathrm{kgU} \text {, final }\end{array}$ & $\begin{array}{l}\text { Exposure } \\
\mathrm{Mwd} / \mathrm{MT}\end{array}$ & $\begin{array}{l}\text { Iifetime, } \\
\text { days at } \\
\text { full power }\end{array}$ & $\frac{\mathrm{kgU} \text { final }}{\mathrm{kgU} \text { initial }}$ \\
\hline \multirow[t]{3}{*}{ A } & 100 & 3.0 & 0.784 & 6.98 & 24,910 & 2080 & .9656 \\
\hline & 300 & 3.0 & 0.732 & 7.11 & 25,920 & 2165 & .9644 \\
\hline & 500 & 3.0 & 0.720 & 7.17 & 26,160 & 2185 & .9640 \\
\hline \multirow[t]{3}{*}{ B } & 100 & 3.5 & 0.856 & 7.54 & 29,400 & 2455 & .9601 \\
\hline & 300 & 3.5 & 0.800 & 7.65 & 30,470 & 2545 & .9587 \\
\hline & 500 & $3 \cdot 5$ & 0.788 & 7.68 & 30,710 & 2565 & .9584 \\
\hline \multirow[t]{3}{*}{ C } & 100 & 4.0 & 0.933 & 8.04 & 33,710 & 2815 & .9547 \\
\hline & 300 & 4.0 & 0.873 & 8.15 & 34,840 & 2910 & .9533 \\
\hline & 500 & 4.0 & 0.861 & 8.17 & 35,080 & 2930 & .9530 \\
\hline \multirow[t]{3}{*}{ D } & 100 & $3 \cdot 5$ & 0.856 & $7 \cdot 54$ & 29,400 & 2455 & .9601 \\
\hline & 300 & 3.5 & 0.800 & 7.65 & 30,470 & 2545 & .9587 \\
\hline & 500 & $3 \cdot 5$ & 0.788 & 7.68 & 30,710 & 2565 & .9584 \\
\hline \multirow[t]{3}{*}{$\mathrm{E}$} & 100 & $3 \cdot 5$ & 0.856 & 7.54 & 29,400 & 2455 & .9601 \\
\hline & 300 & 3.5 & 0.800 & 7.65 & 30,470 & 2545 & .9587 \\
\hline & 500 & 4.0 & 0.861 & 8.17 & 35,080 & 2930 & .9530 \\
\hline
\end{tabular}


TABLE A-6

COST PARAMETERS OF OPTIMUM CASES FOR BOILING

WATER REACTORS FUELED WITH ENRICHED URANIUM

Use Charge on Uranium and Plutonium: 12\%

\begin{tabular}{|c|c|c|c|c|c|c|c|}
\hline $\begin{array}{l}\text { Cost } \\
\text { Sched- } \\
\text { ule }\end{array}$ & $\begin{array}{l}\text { Power } \\
\text { Leve 1, } \\
\text { Mwe }\end{array}$ & $\begin{array}{l}\text { Initial } \\
\text { wt. \% } \\
U-235\end{array}$ & $\begin{array}{l}\text { Final } \\
\text { wt. \% } \\
\text { U-235 } \\
\end{array}$ & $\begin{array}{l}\text { Final Pu } \\
\mathrm{gm} / \mathrm{kgU} \text {, final }\end{array}$ & $\begin{array}{l}\text { Exposure } \\
\text { Mwd/MT }\end{array}$ & $\begin{array}{l}\text { Lifetime, } \\
\text { days at } \\
\text { full power }\end{array}$ & $\begin{array}{l}\mathrm{kgU} \text { final } \\
\mathrm{KgU} \text { initial }\end{array}$ \\
\hline \multirow[t]{3}{*}{ A } & 100 & 2.5 & 0.720 & 6.36 & 20,180 & 1685 & .9715 \\
\hline & 300 & 2.5 & 0.670 & 6.48 & 21,130 & 1765 & .9703 \\
\hline & 500 & 2.25 & 0.628 & 6.20 & 18,920 & 1580 & .9731 \\
\hline \multirow[t]{3}{*}{ B } & 100 & 3.0 & 0.784 & 6.98 & 24,910 & 2080 & .9656 \\
\hline & 300 & 2.5 & 0.670 & 6.48 & 21,130 & 1765 & .9703 \\
\hline & 500 & 2.5 & 0.658 & 6.52 & 21,370 & 1785 & .9700 \\
\hline \multirow[t]{3}{*}{$\mathrm{C}$} & 100 & 3.0 & 0.784 & 6.98 & 24,910 & 2080 & .9656 \\
\hline & 300 & 3.0 & 0.732 & 7.11 & 25,920 & 2165 & .9644 \\
\hline & 500 & 3.0 & 0.720 & 7.17 & 26,160 & 2185 & .9640 \\
\hline \multirow[t]{3}{*}{$D$} & 100 & 3.0 & 0.784 & 6.98 & 24,910 & 2080 & .9656 \\
\hline & 300 & 2.5 & 0.670 & 6.48 & 21,130 & 1765 & .9703 \\
\hline & 500 & 2.5 & 0.658 & 6.52 & 21,370 & 1785 & .9700 \\
\hline \multirow[t]{3}{*}{$E$} & 100 & 3.0 & 0.784 & 6.98 & 24,910 & 2080 & .9656 \\
\hline & 300 & 3.0 & 0.732 & 7.11 & 25,920 & 2165 & .9644 \\
\hline & 500 & 3.0 & 0.720 & $7 \cdot 17$ & 26,160 & 2185 & .9640 \\
\hline
\end{tabular}


TABLE A-T

\section{COST PARAMETERS OF OPTIMUM CASES FOR BOILING WATER}

REACTORS FUEIED WITH U-235 IN THORTUM

Use Charge on Uranium: $4-3 / 4 \%$

\begin{tabular}{|c|c|c|c|c|c|c|c|c|}
\hline $\begin{array}{l}\text { Cost } \\
\text { Sched- } \\
\text { ule }\end{array}$ & $\begin{array}{l}\text { Power } \\
\text { Leve1, } \\
\text { Mwe }\end{array}$ & $\begin{array}{l}\text { Initial } \\
\text { wt. \% } \\
\text { U-235 } \\
\text { in fuel }\end{array}$ & $\begin{array}{l}\text { Final } \\
\text { wt. \% U } \\
\text { in fuel }\end{array}$ & $\begin{array}{l}\text { wt. } \% \\
\mathrm{U}-235 \\
\text { in } \mathrm{Fi}- \\
\text { nal U }\end{array}$ & $\begin{array}{l}\text { wt. \% } \\
\text { U-233 } \\
\text { in final } \\
\text { U }\end{array}$ & $\begin{array}{l}\text { Final } \\
\mathrm{kg} \text { fuel } \\
\text { initial } \\
\mathrm{kg} \text { fuel }\end{array}$ & $\begin{array}{l}\text { Expo- } \\
\text { sure } \\
\text { Mwd/MT }\end{array}$ & $\begin{array}{l}\text { Lifetime, } \\
\text { days at } \\
\text { full } \\
\text { power }\end{array}$ \\
\hline \multirow[t]{3}{*}{ A } & 100 & 3.0 & 2.544 & 29.82 & 48.00 & .9706 & 26,910 & 2260 \\
\hline & 300 & 3.0 & 2.527 & 26.21 & 50.11 & .9680 & 29,350 & 2465 \\
\hline & 500 & 3.0 & 2.523 & 25.33 & 50.60 & .9671 & 30,000 & 2520 \\
\hline \multirow[t]{3}{*}{ B } & 100 & $3 \cdot 5$ & 2.740 & 27.97 & 47.02 & .9639 & 32,930 & 2765 \\
\hline & 300 & 3.0 & 2.527 & 26.21 & 50.1 .1 & .9680 & 29,350 & 2465 \\
\hline & 500 & 3.0 & 2.523 & 25.33 & 50.60 & .9671 & 30,000 & 2520 \\
\hline \multirow[t]{3}{*}{ C } & 100 & 3.75 & 2.837 & 27.48 & 46.34 & .9609 & 35,670 & 2995 \\
\hline & 300 & $3 \cdot 5$ & 2.719 & 25.06 & 48.62 & .9614 & 35,190 & 2955 \\
\hline & 500 & $3 \cdot 5$ & 2.714 & $24 \cdot 34$ & 49.00 & .9608 & 35,790 & 3005 \\
\hline \multirow[t]{3}{*}{ D } & 100 & 3.25 & 2.643 & 28.64 & 47.64 & .9671 & 30,070 & 2525 \\
\hline & 300 & 3.0 & 2.527 & 26.21 & 50.11 & .9680 & 29,350 & 2465 \\
\hline & 500 & 3.0 & 2.523 & $25 \cdot 33$ & 50.60 & .9671 & 30,000 & 2520 \\
\hline \multirow[t]{3}{*}{$\mathrm{E}$} & 100 & 3.25 & 2.643 & 28.64 & 47.64 & .9671 & 30,070 & 2525 \\
\hline & 300 & 3.0 & 2.527 & 26.21 & 50.11 & .9680 & 29,350 & 2465 \\
\hline & 500 & 3.0 & 2.523 & 25.33 & 50.60 & .9671 & 30,000 & 2520 \\
\hline
\end{tabular}


TABLE A-8

COST PARAMETERS OF NEAR-OPTIMUM CASES FOR BOILING

WATER REACTORS FUELED WITH U-235 IN THORIUM

Use Charge on Uranium: $12 \%$

\begin{tabular}{|c|c|c|c|c|c|c|c|c|}
\hline $\begin{array}{l}\text { Cost } \\
\text { Sched- } \\
\text { ule }\end{array}$ & $\begin{array}{l}\text { Power } \\
\text { Level, } \\
\text { Mwe }\end{array}$ & $\begin{array}{l}\text { Initial } \\
\text { wt. \% } \\
\text { U- } 235 \\
\text { in fuel }\end{array}$ & $\begin{array}{l}\text { Final } \\
\text { wt. o } \mathrm{U} \\
\text { in fuel }\end{array}$ & $\begin{array}{l}\text { wt. } \% \\
\text { U-235 } \\
\text { in Fi- } \\
\text { nal U }\end{array}$ & $\begin{array}{l}\text { wt. } \% \\
U-233 \\
\text { in Fi- } \\
\text { nal U }\end{array}$ & $\begin{array}{l}\text { Final } \\
\mathrm{kg} \text { fuel } \\
\text { initial } \\
\mathrm{kg} \text { fuel }\end{array}$ & $\begin{array}{l}\text { Expo- } \\
\text { sure } \\
\text { Mwd/MT }\end{array}$ & $\begin{array}{l}\text { Lifetime, } \\
\text { days at } \\
\text { full } \\
\text { power } \\
\end{array}$ \\
\hline \multirow[t]{3}{*}{ A } & 100 & 3.0 & 2.544 & 29.82 & 48.00 & .9706 & 26,910 & 2260 \\
\hline & 300 & 2.5 & 2.317 & 29.95 & 50.16 & .9759 & 22,090 & 1855 \\
\hline & 500 & 2.5 & 2.315 & 28.61 & 50.98 & .9751 & 22,850 & 1920 \\
\hline \multirow[t]{3}{*}{ B } & 100 & 3.0 & 2.544 & 29.82 & 48.00 & .9706 & 26,910 & 2260 \\
\hline & 300 & 2.75 & 2.422 & 27.61 & 50.51 & .9716 & 25,960 & 2180 \\
\hline & 500 & 2.75 & 2.423 & 26.49 & 51.09 & .9709 & 26,700 & 2240 \\
\hline \multirow[t]{3}{*}{ C } & 100 & 3.25 & 2.643 & 28.64 & 47.64 & .9671 & 30,070 & 2525 \\
\hline & 300 & 3.0 & 2.527 & 26.21 & 50.11 & .9680 & 29,350 & 2465 \\
\hline & 500 & 3.0 & 2.523 & $25 \cdot 33$ & 50.60 & .9671 & 30,000 & 2520 \\
\hline \multirow[t]{3}{*}{ D } & 100 & 3.0 & 2.544 & 29.82 & 48.00 & .9706 & 26,910 & 2260 \\
\hline & 300 & 2.75 & 2.422 & 27.61 & 50.51 & .9716 & 25,960 & $278 n$ \\
\hline & 500 & 2.75 & 2.423 & 26.49 & 51.09 & .9709 & 26,700 & 2240 \\
\hline \multirow[t]{3}{*}{$\mathrm{E}$} & 100 & 2.75 & 2.546 & 30.40 & 45.78 & .9756 & 23,400 & 1965 \\
\hline & 300 & 2.5 & 2.317 & 29.95 & 50.16 & .9759 & 22,090 & 1855 \\
\hline & 500 & 2.5 & 2.315 & 28.61 & 50.98 & .9751 & 22,850 & 1920 \\
\hline
\end{tabular}




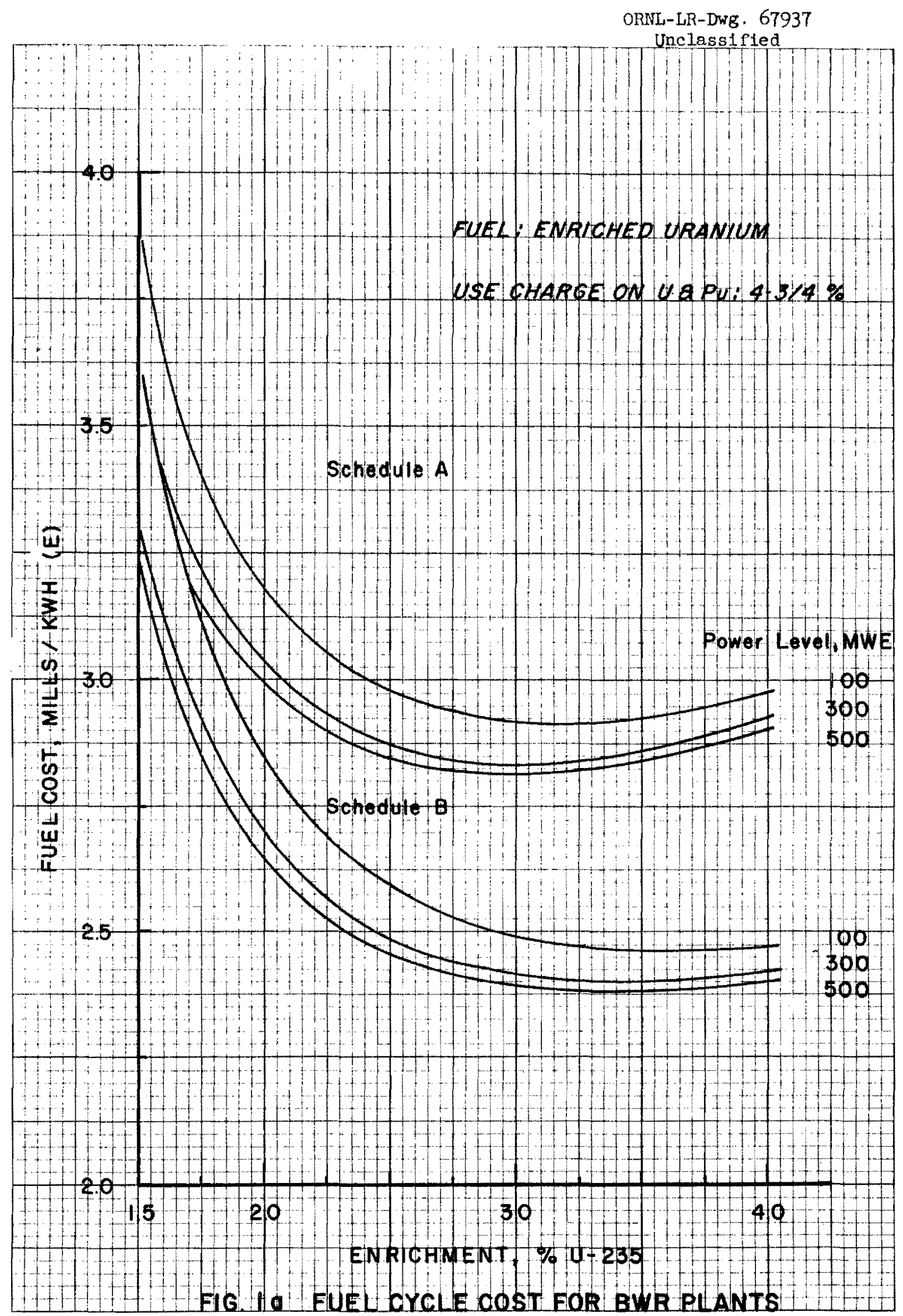




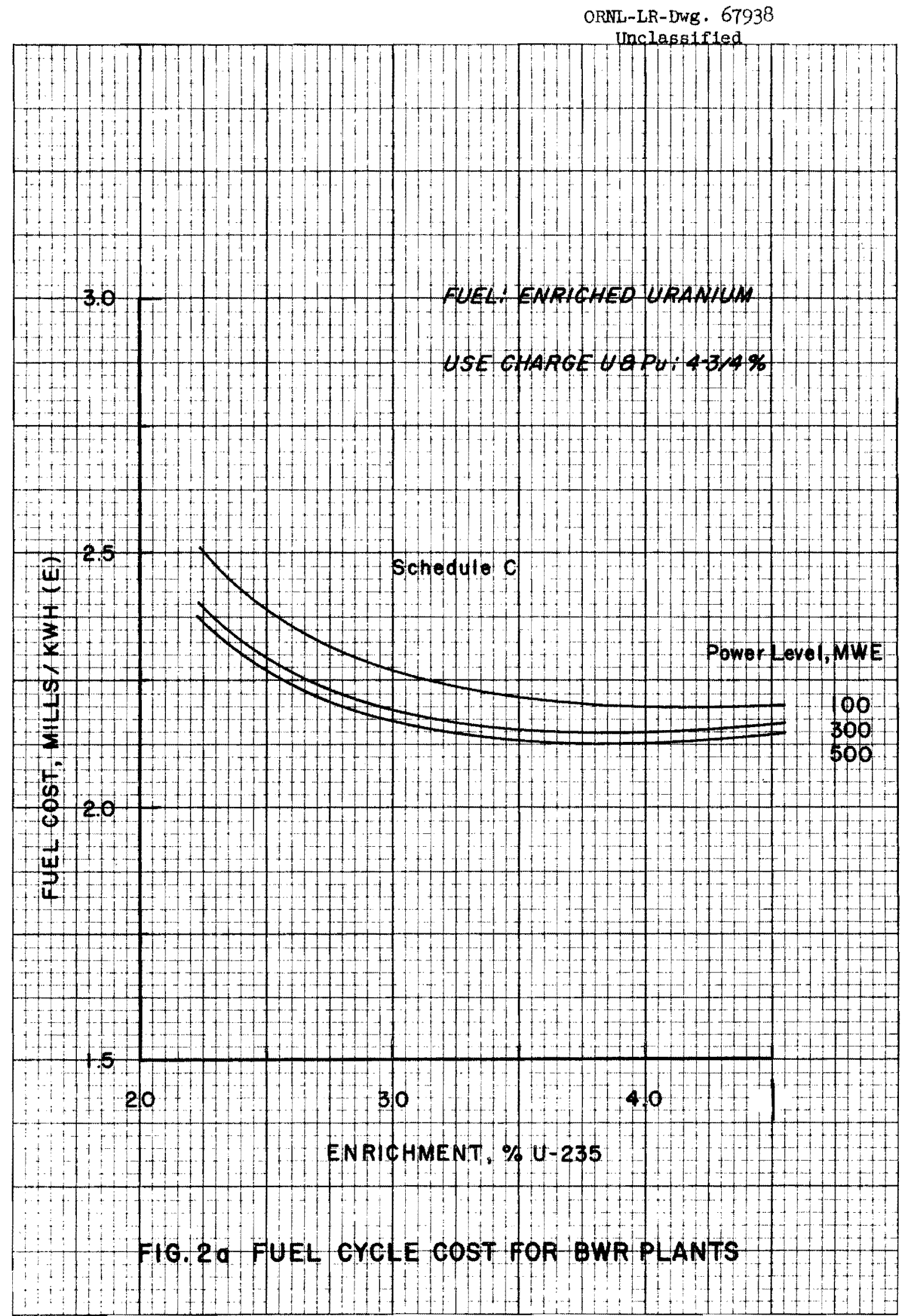


ORNL-LR-DWg . 67939

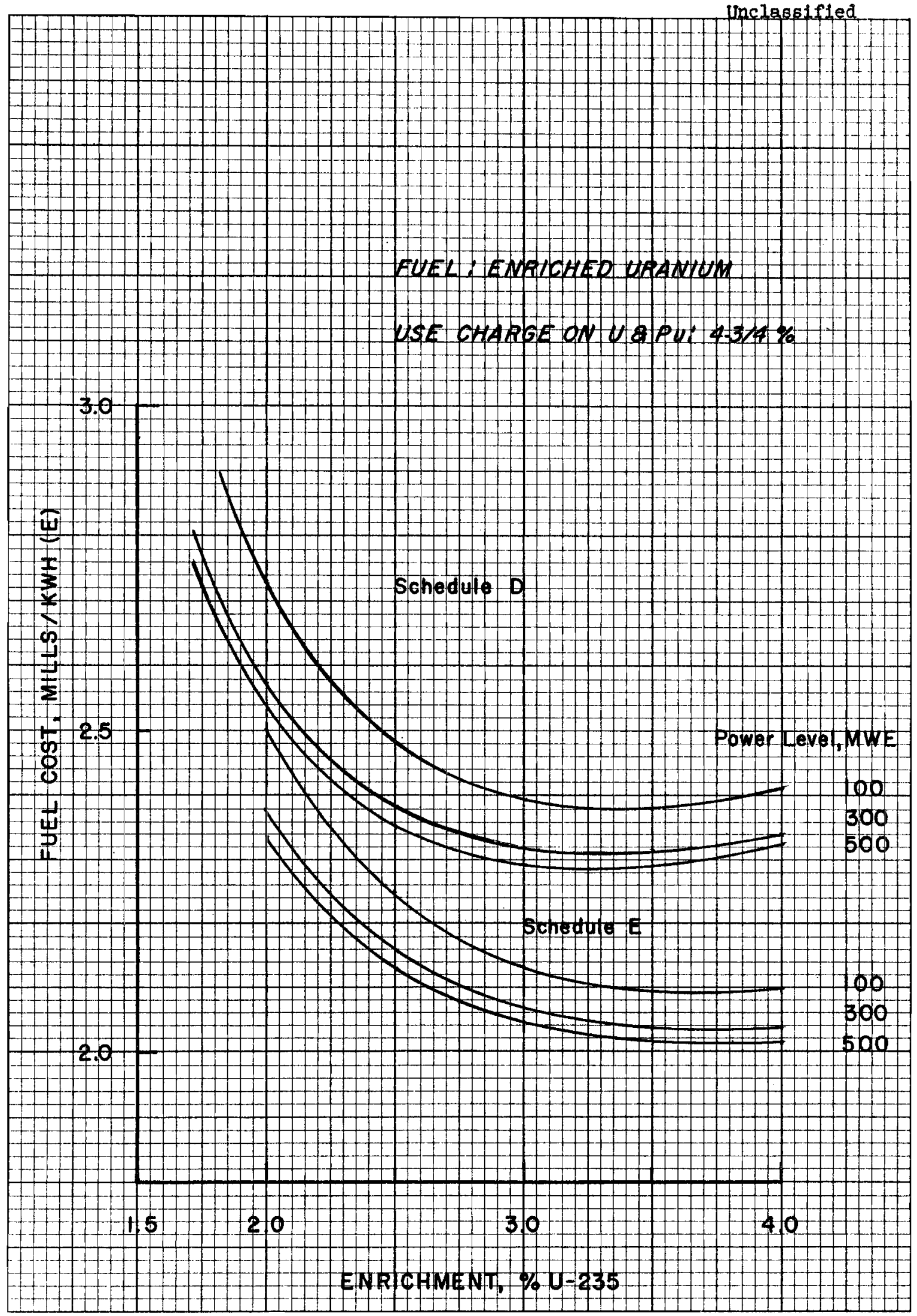

FIG. 3 a FUEL CYCLE COST FOR BWR PLANTS 


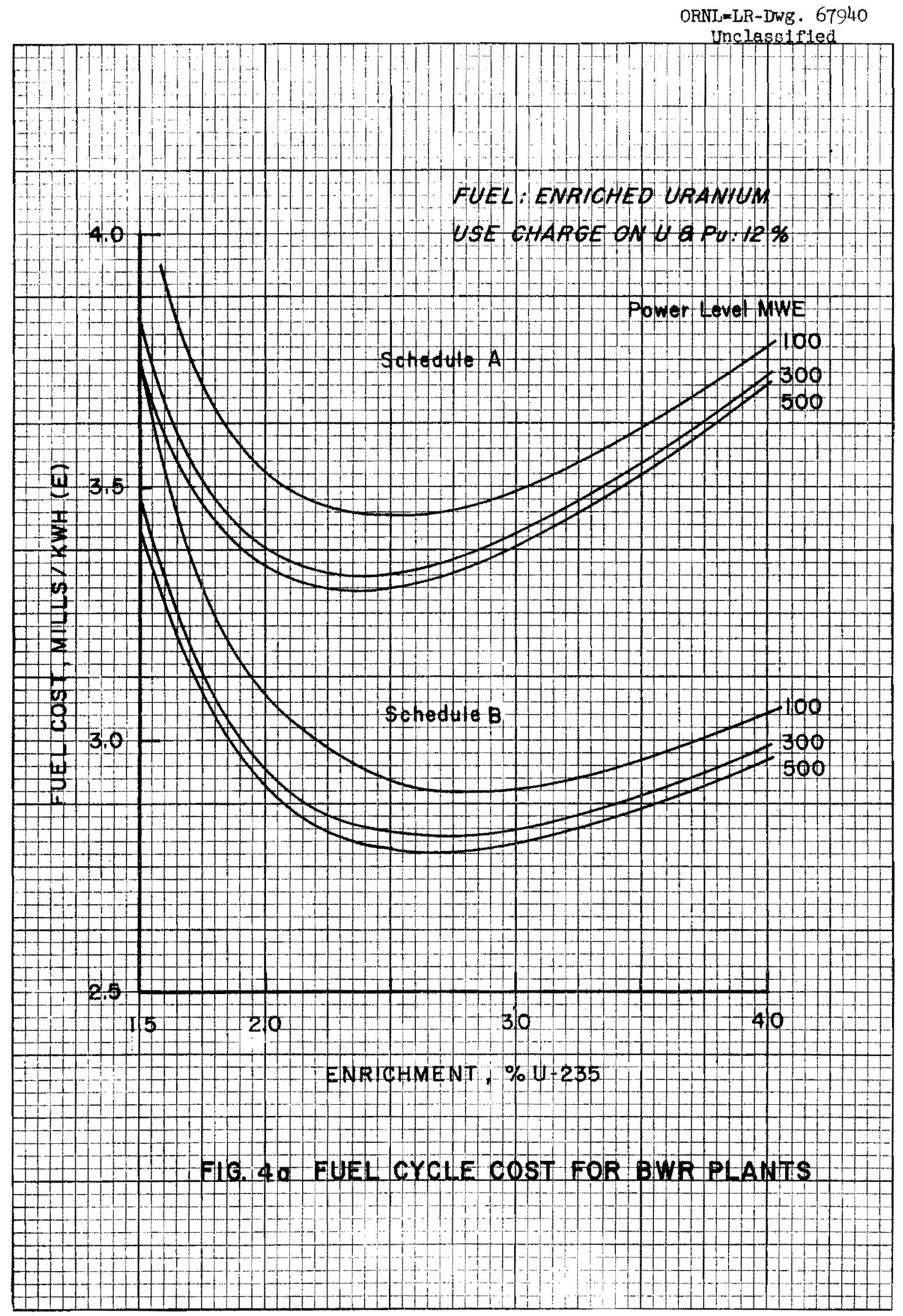




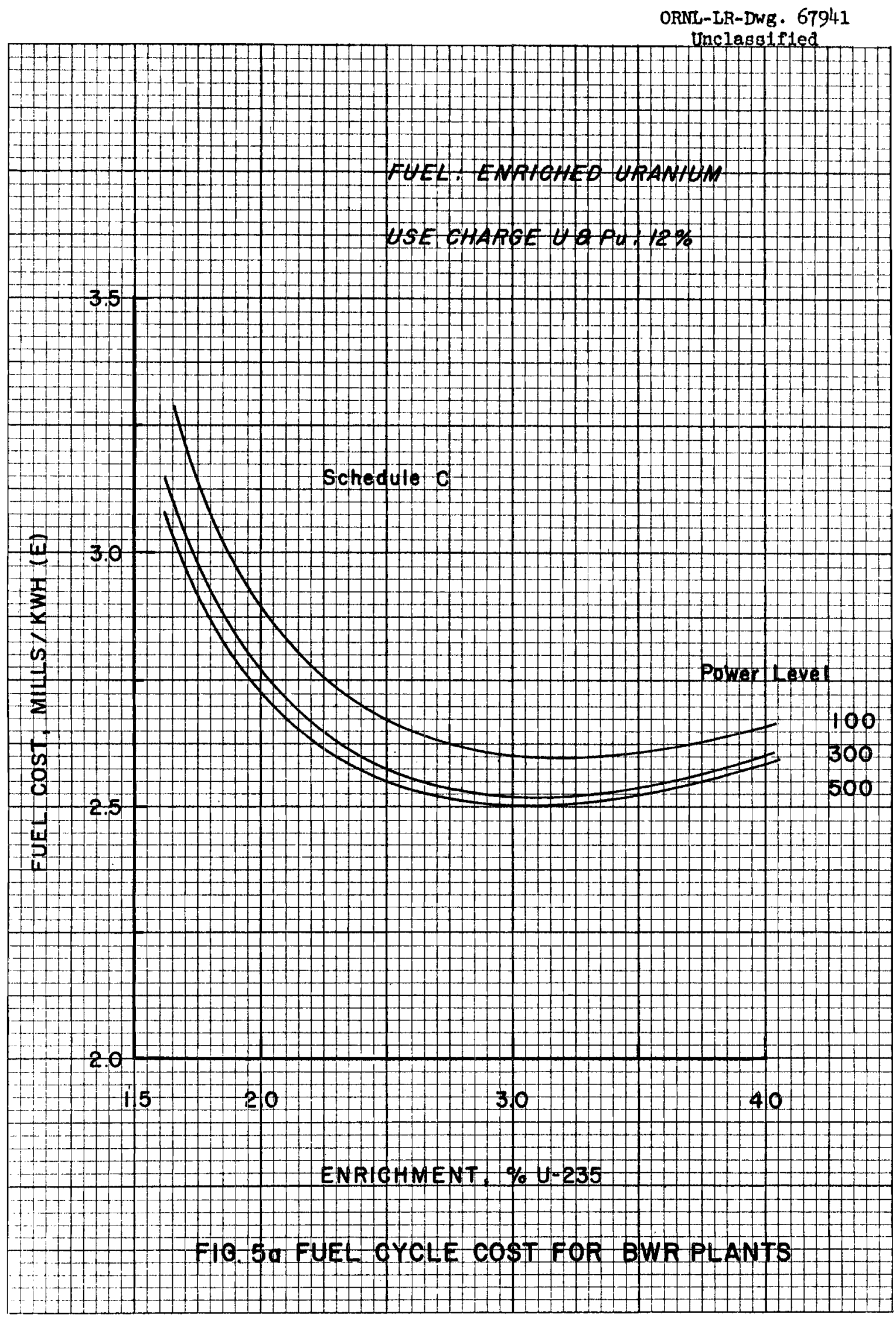




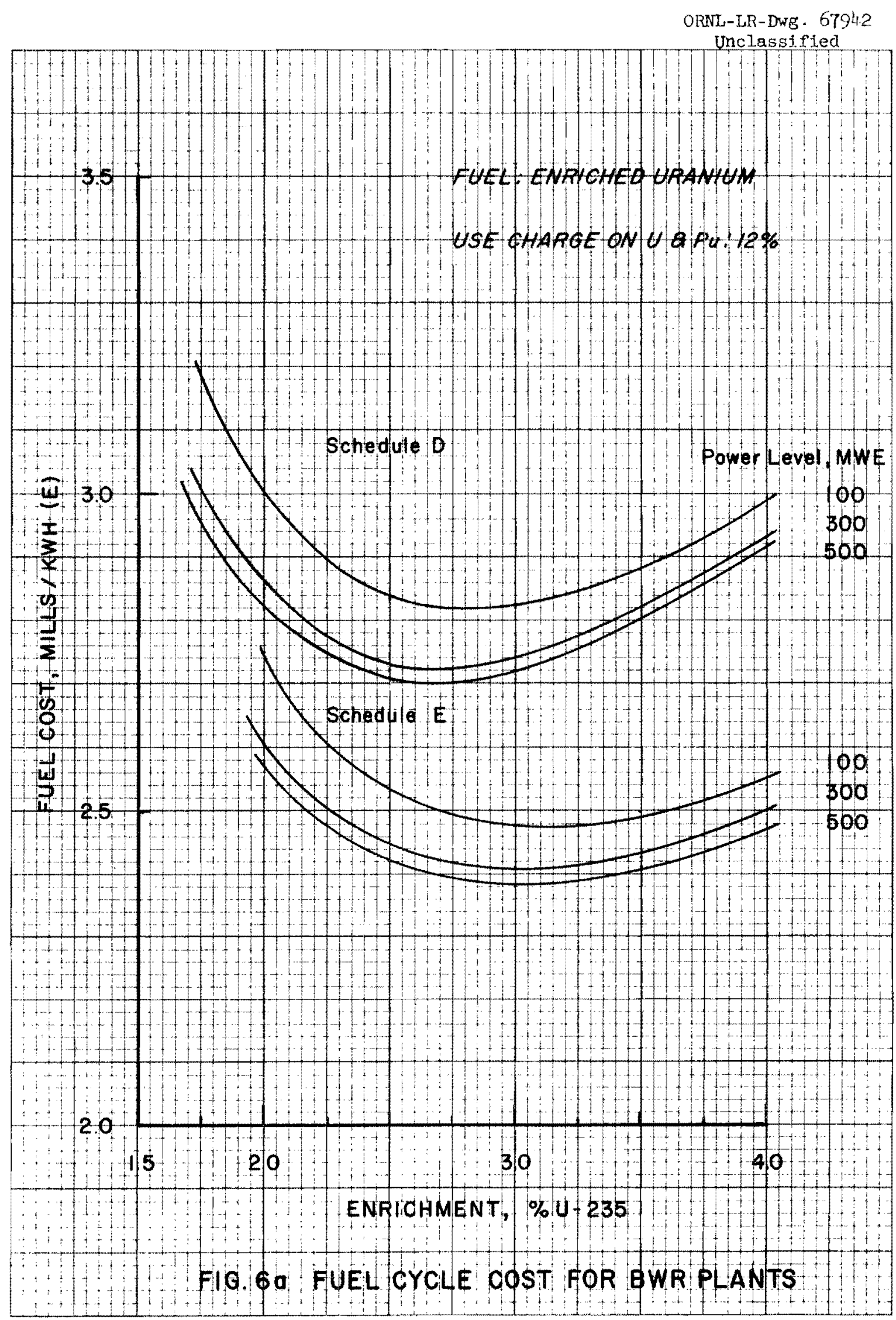


ORNL-LR-DWg. 67943

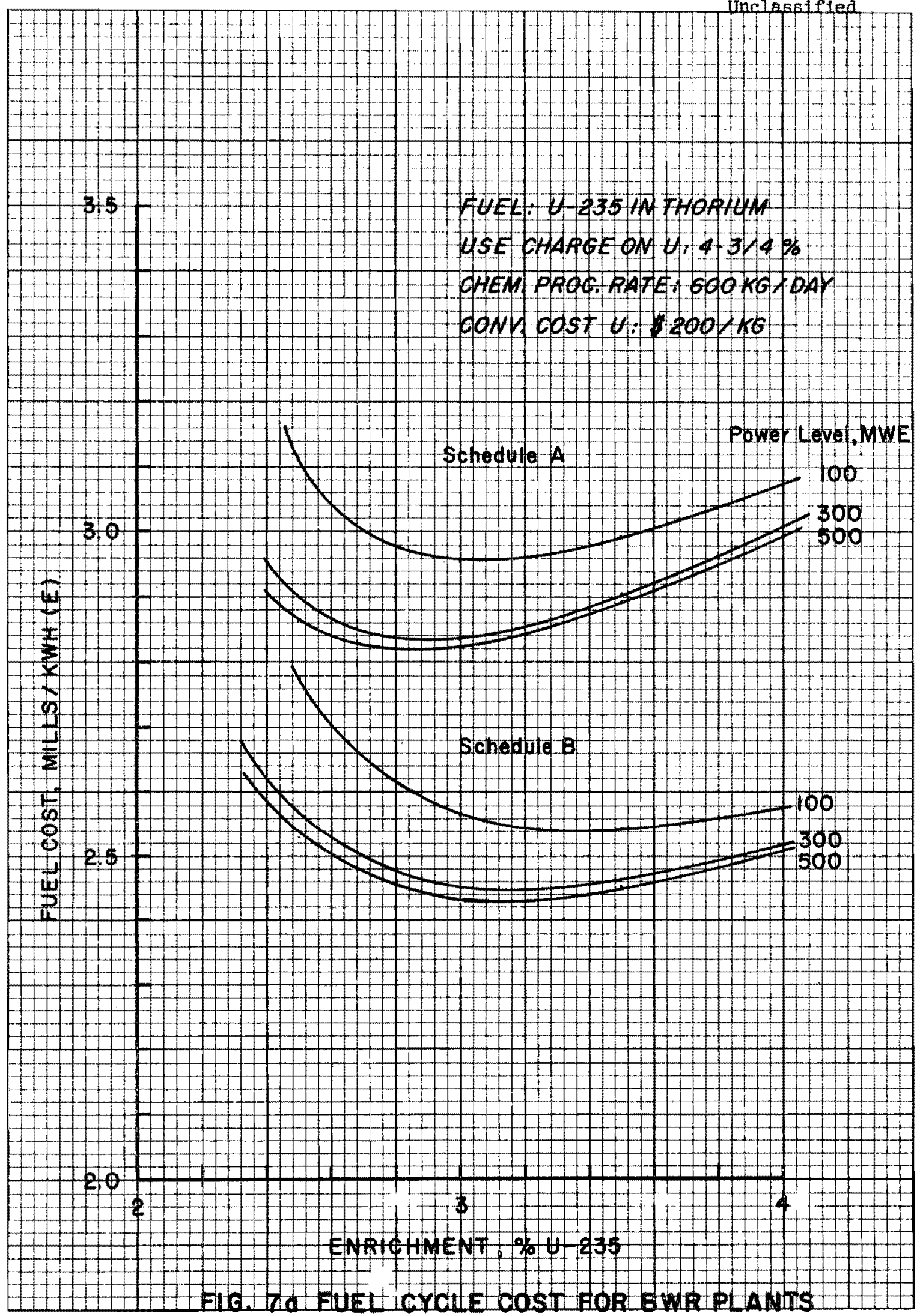


ORNL-LR-DWB . 67944

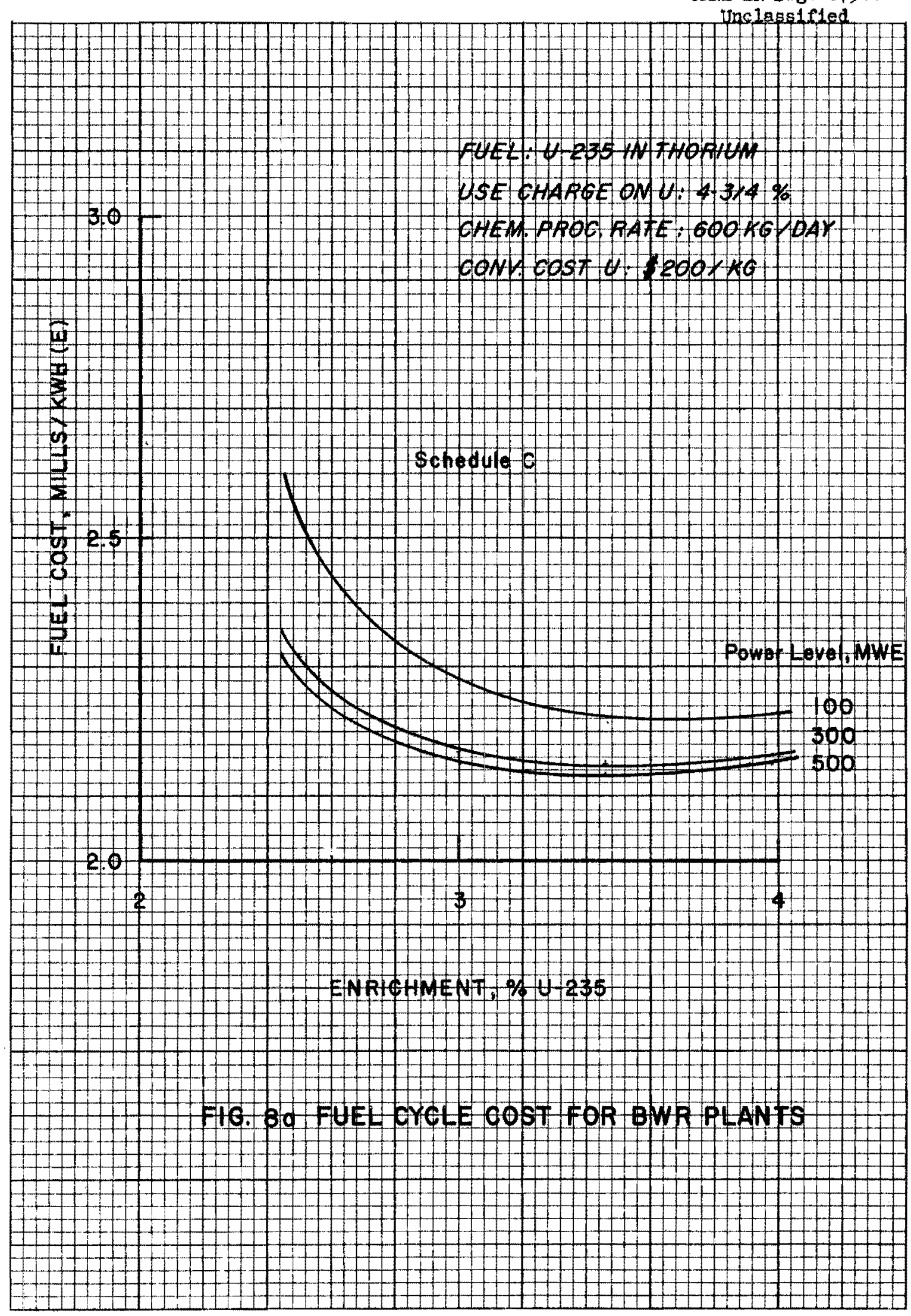


ORNL-LR-Dwg. 67945

Unclasstfled

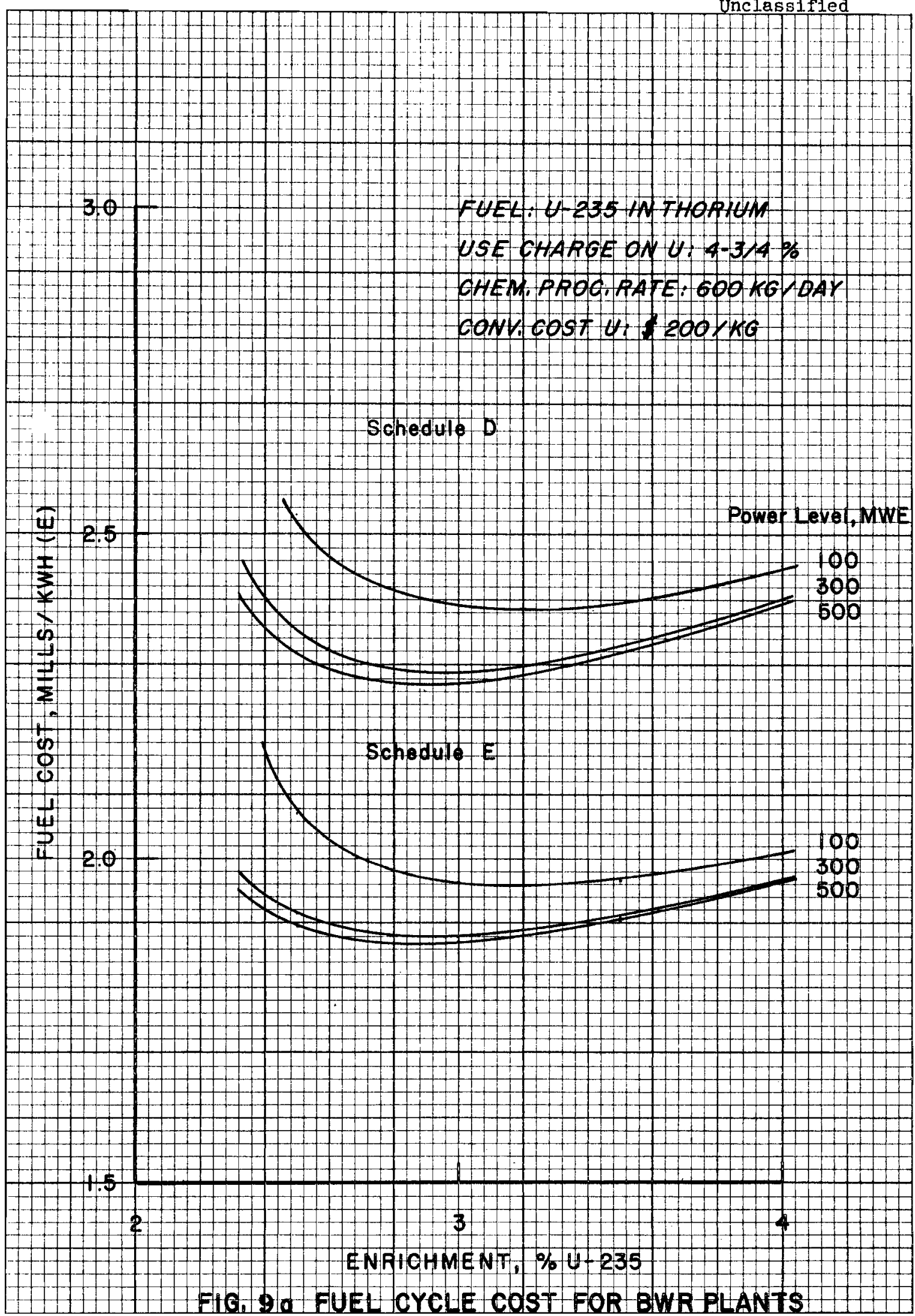


ORRL-LR-Dwg . 6701.6

Unclassified

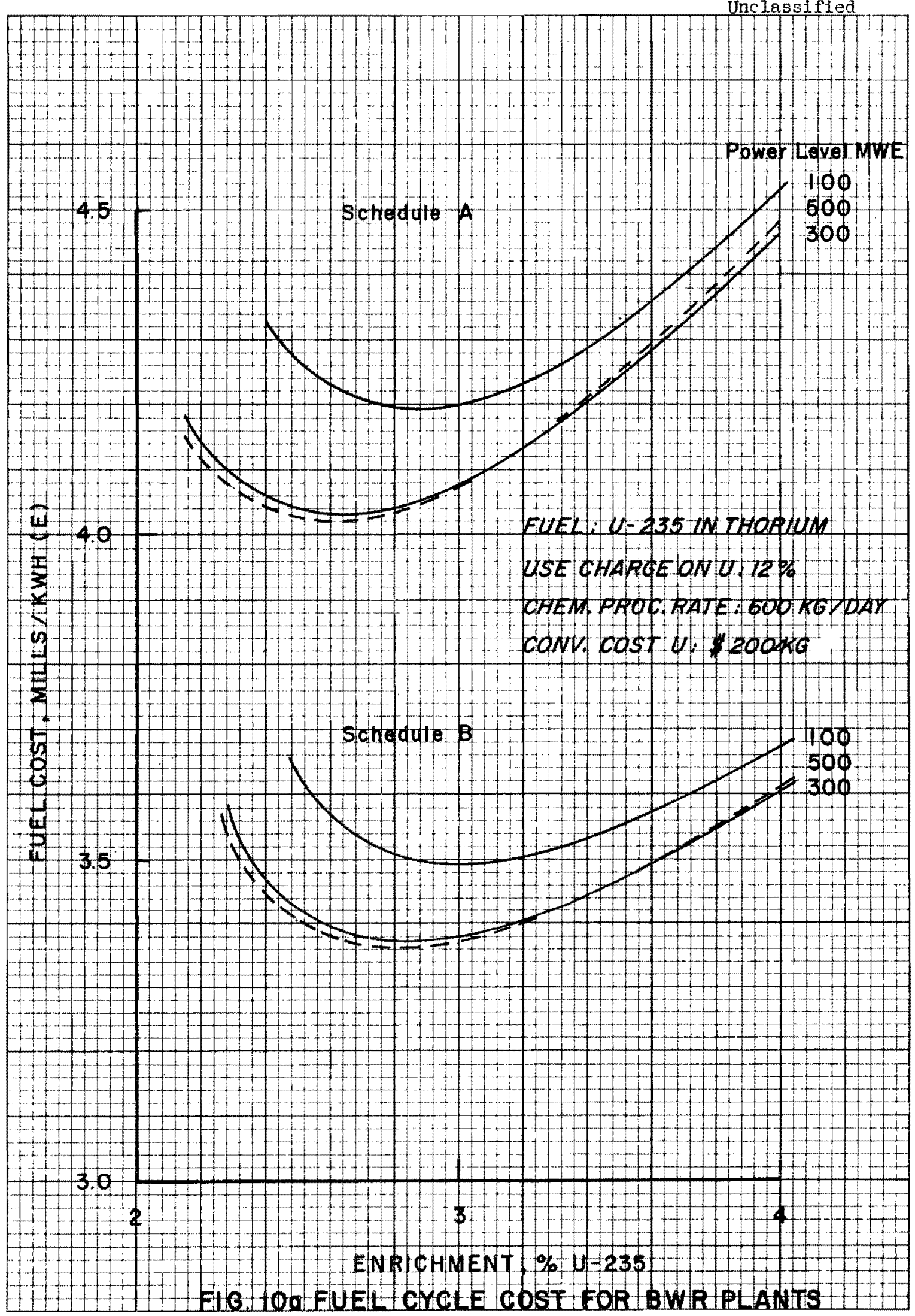




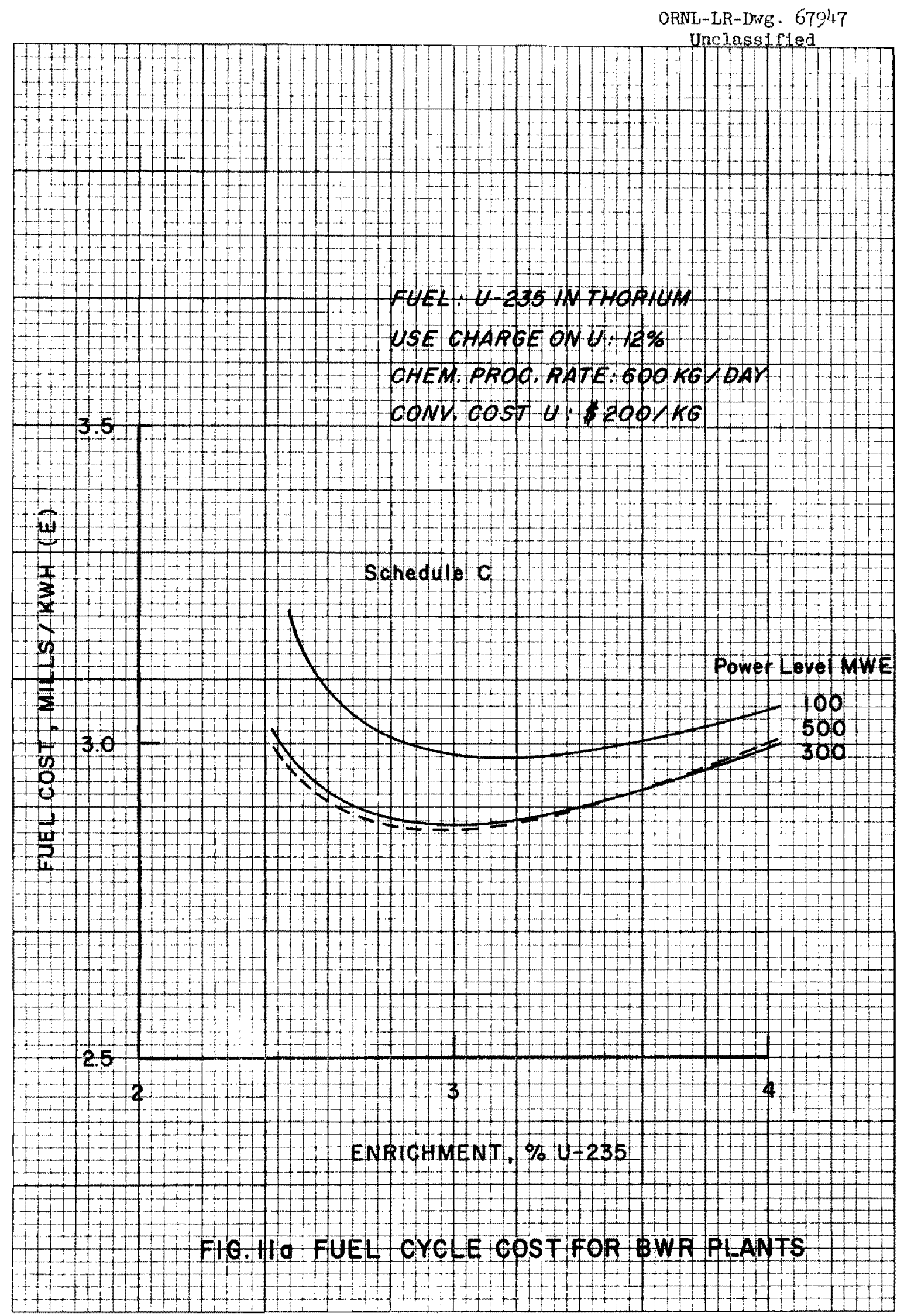


ORNL-IR-DWB . 67948

Unclassifled

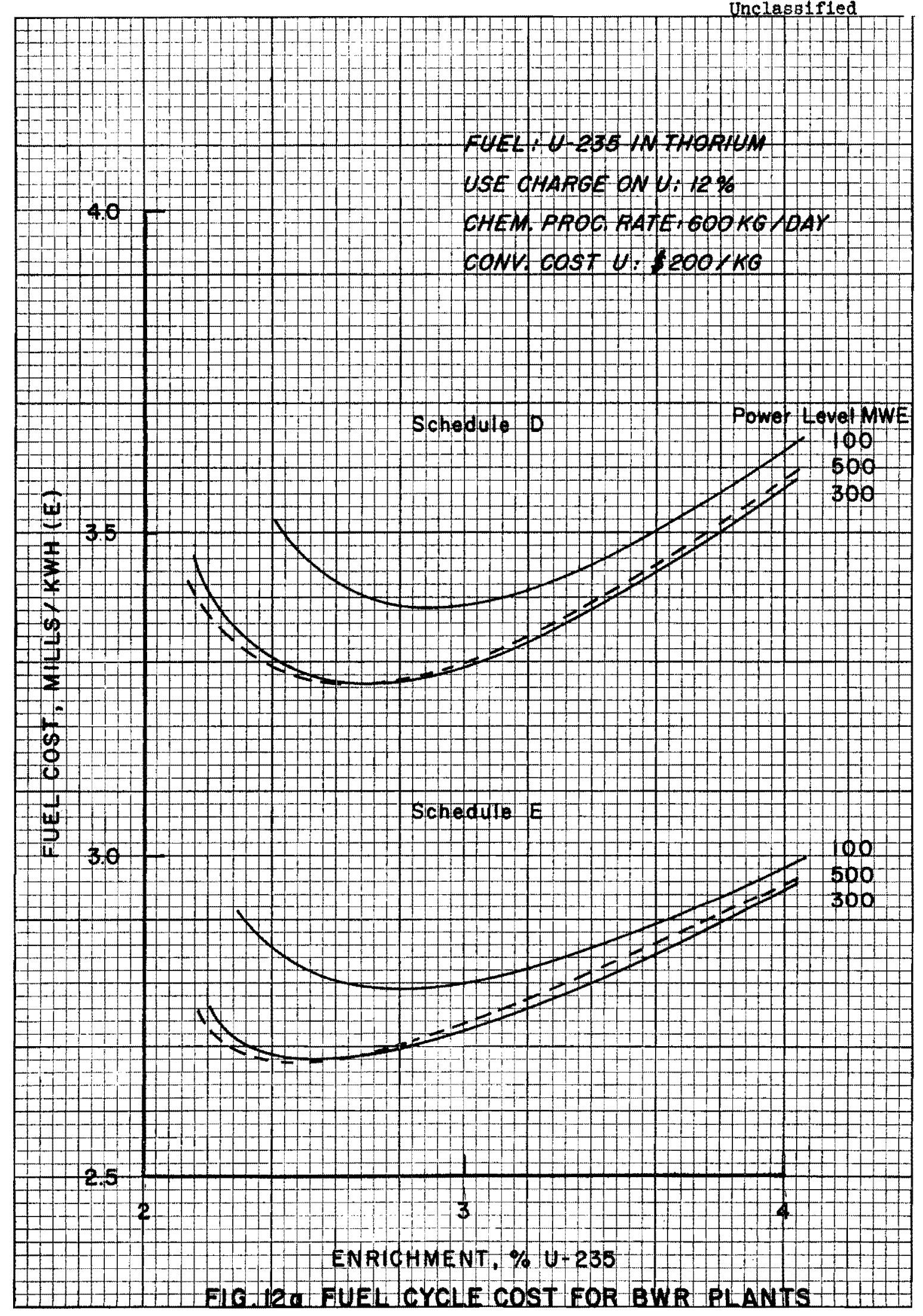


ORNL-LR-DWg. 67949

Unclassteled

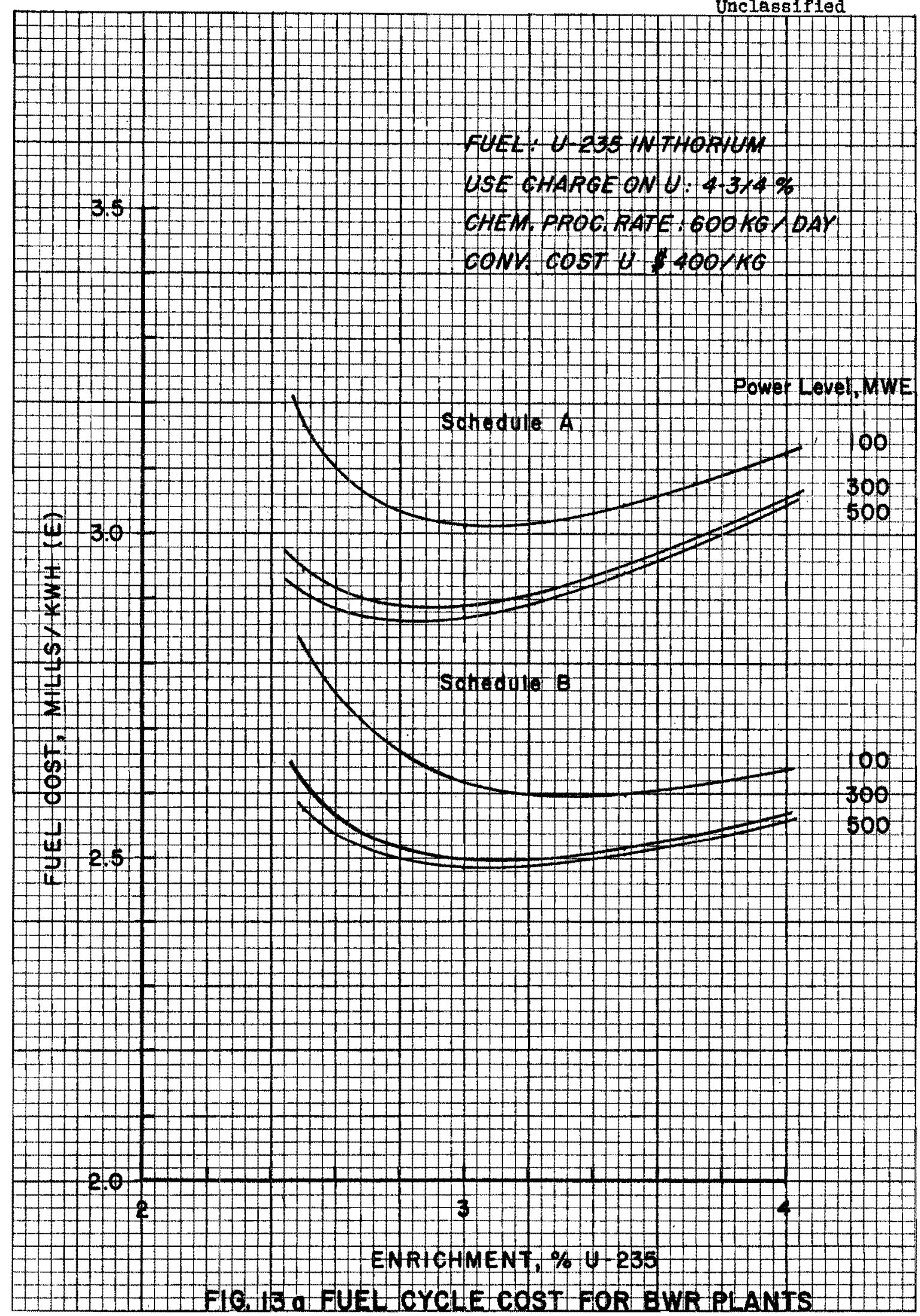


ORNL-LR-DWg. 67950

unclassifled

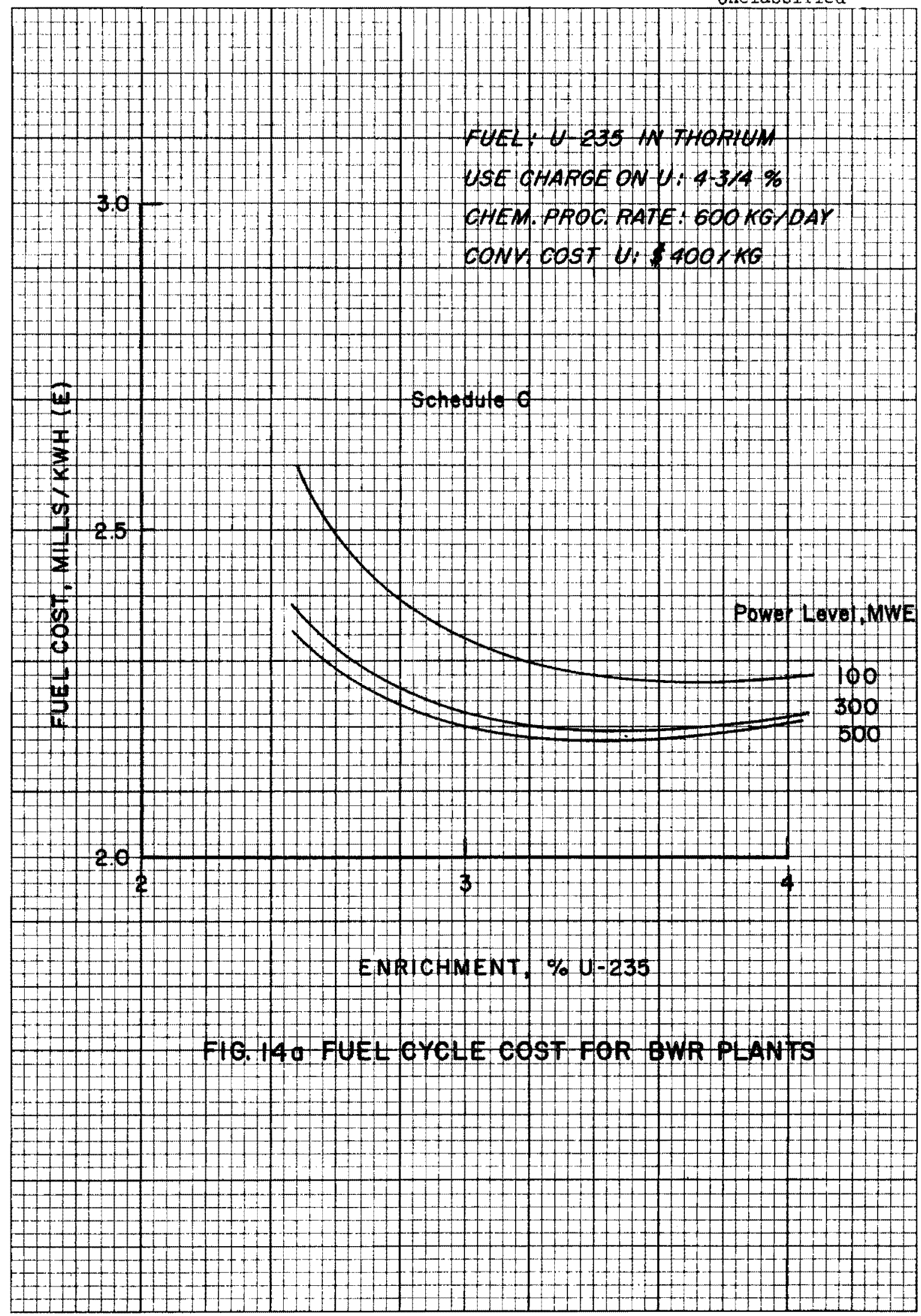


ORRL-LR-DWB . 67951

Unclasstified

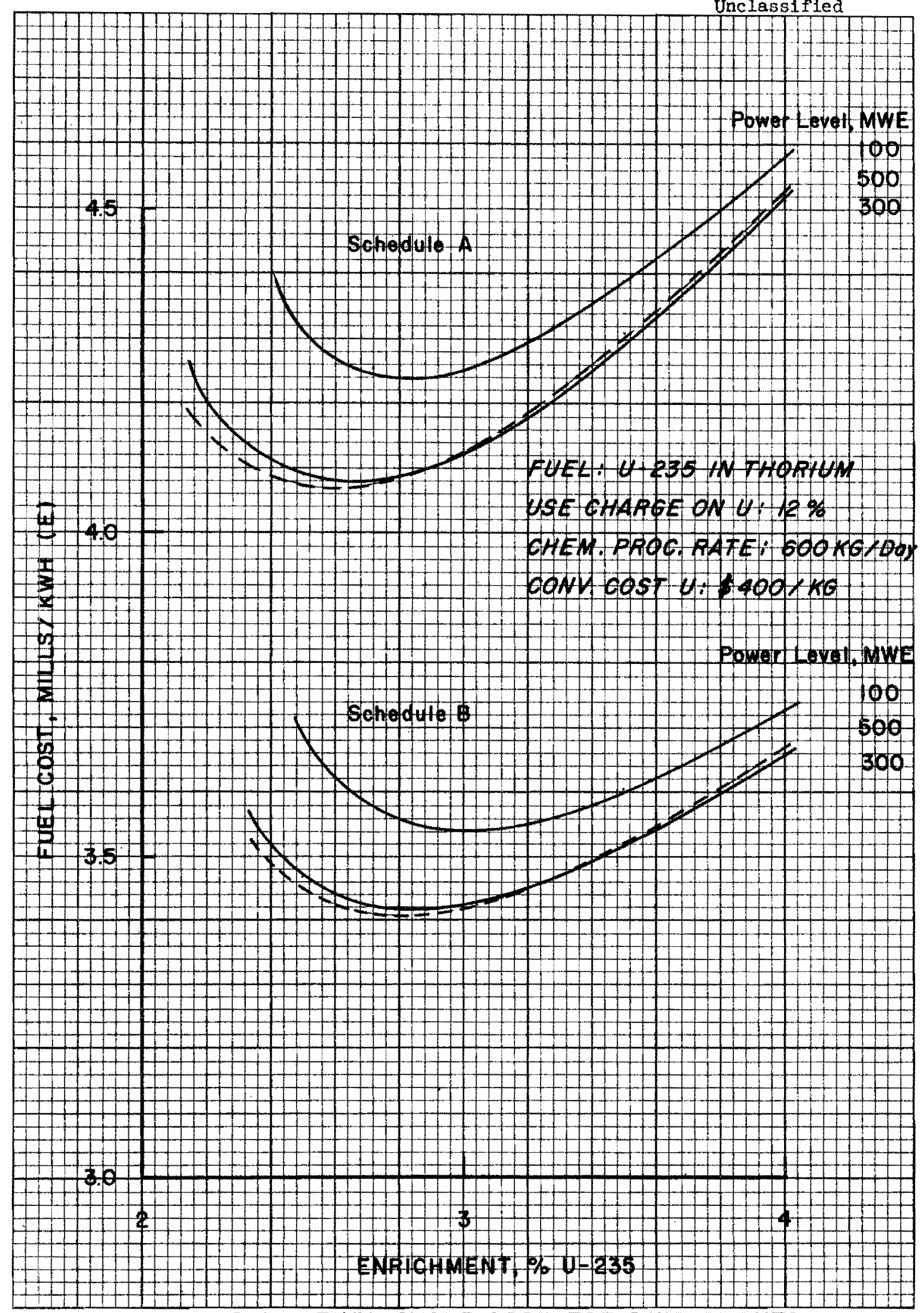

FIG. I5a FUEL CYCLE COST FOR BWR PLANTS 
ORNL-LR-Dwg. 67952

Unclassteded

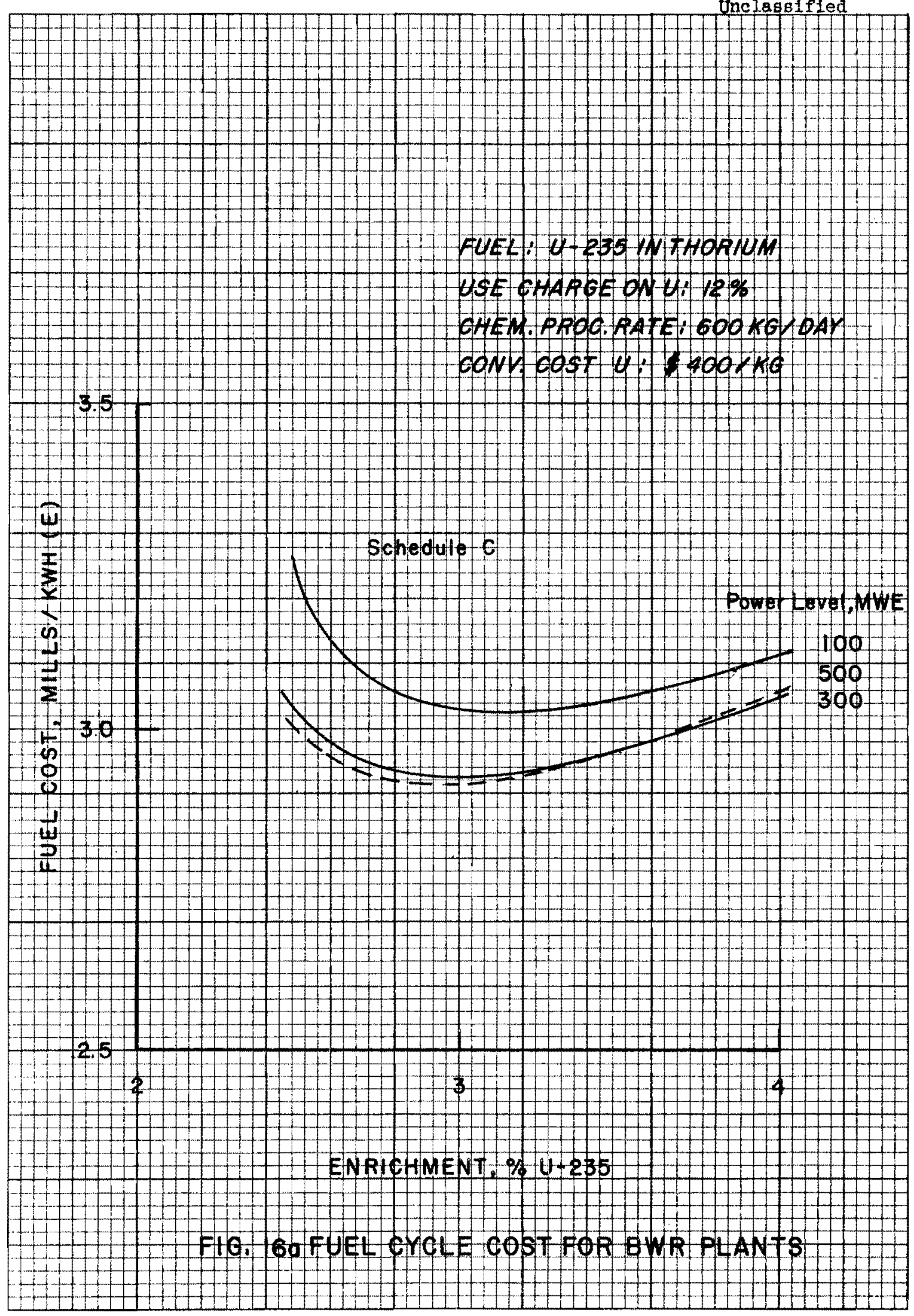


ORNL-IR-DWg 67953

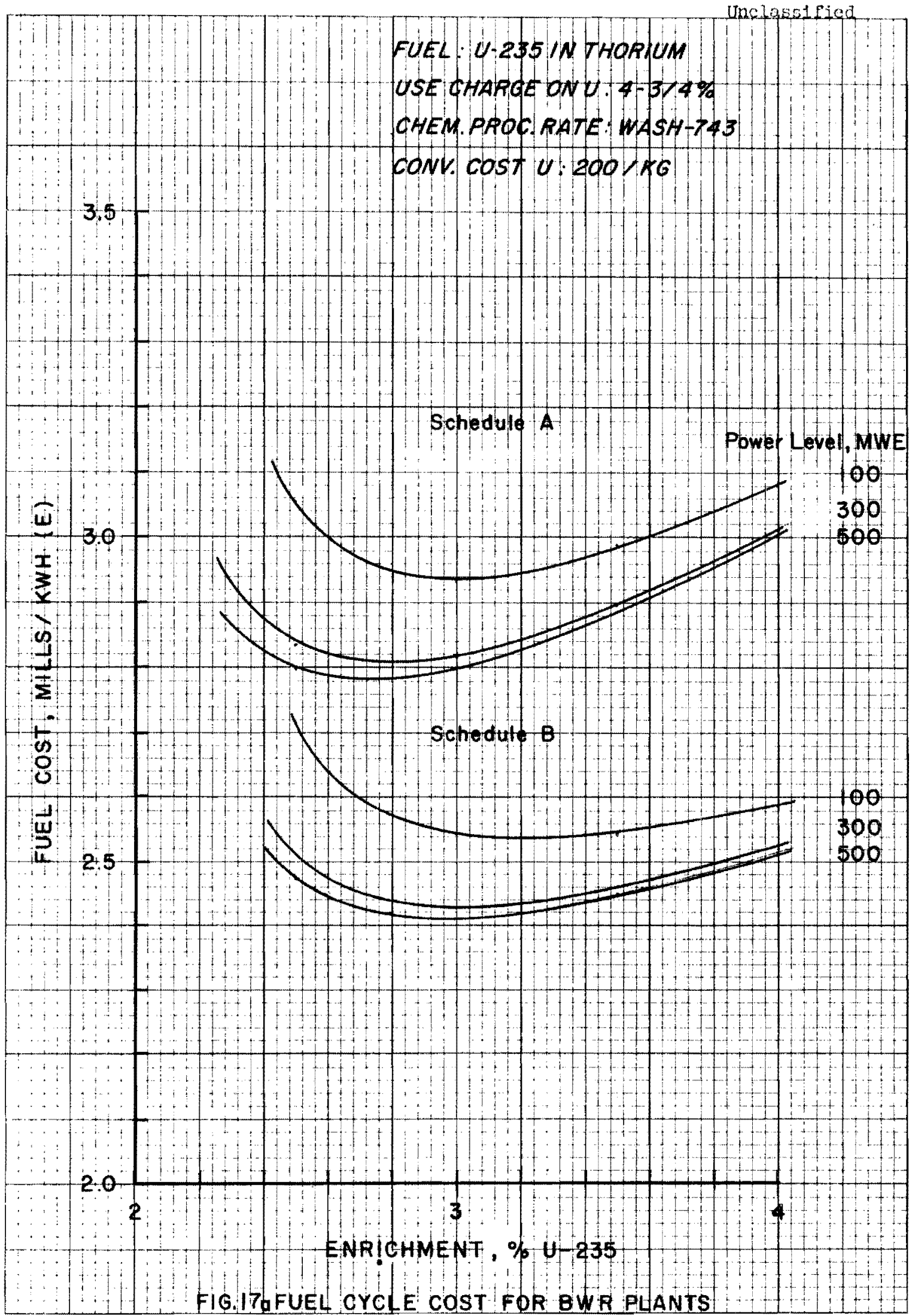


ORNL-LR-DWg. 67954

Unclassified

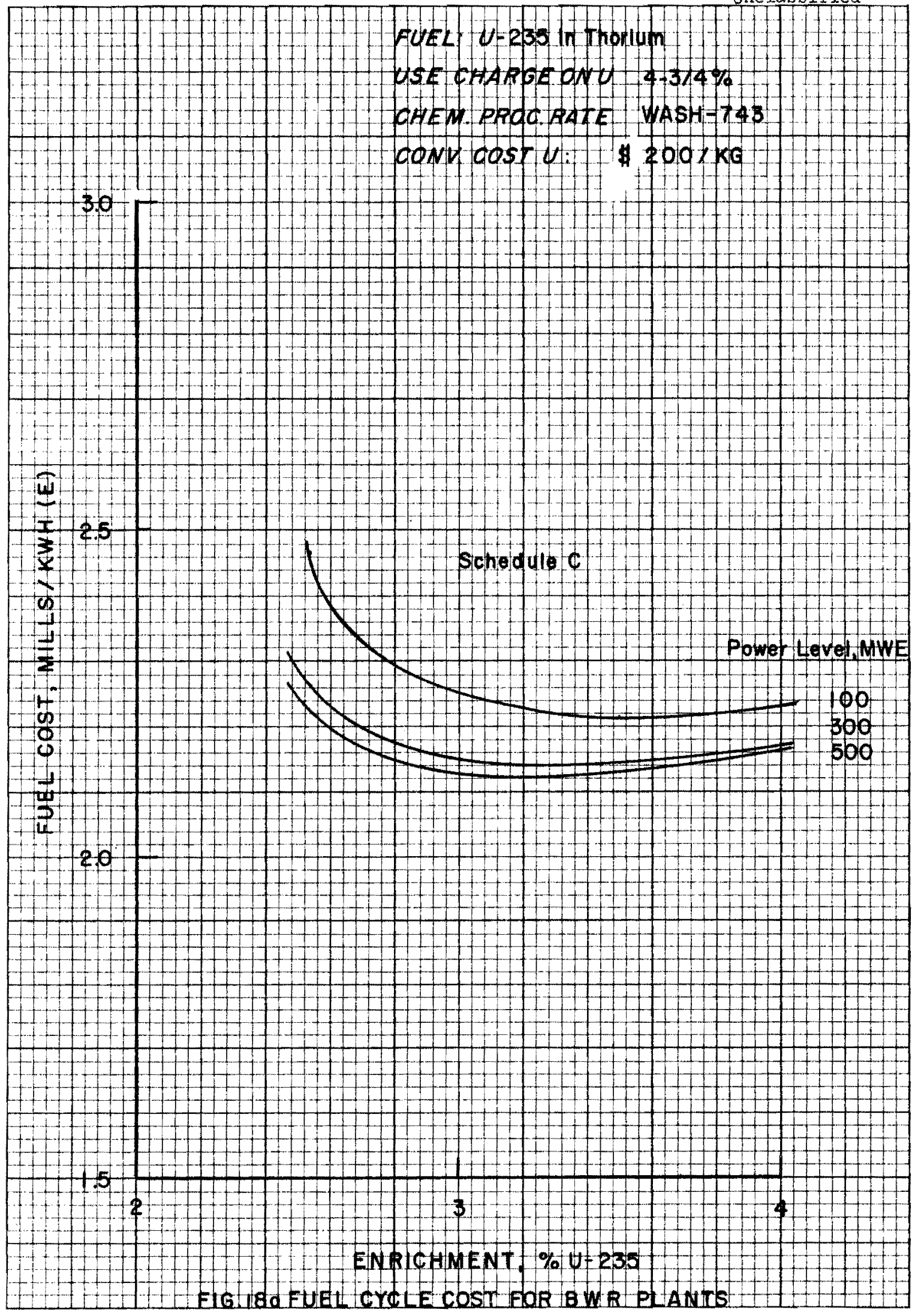




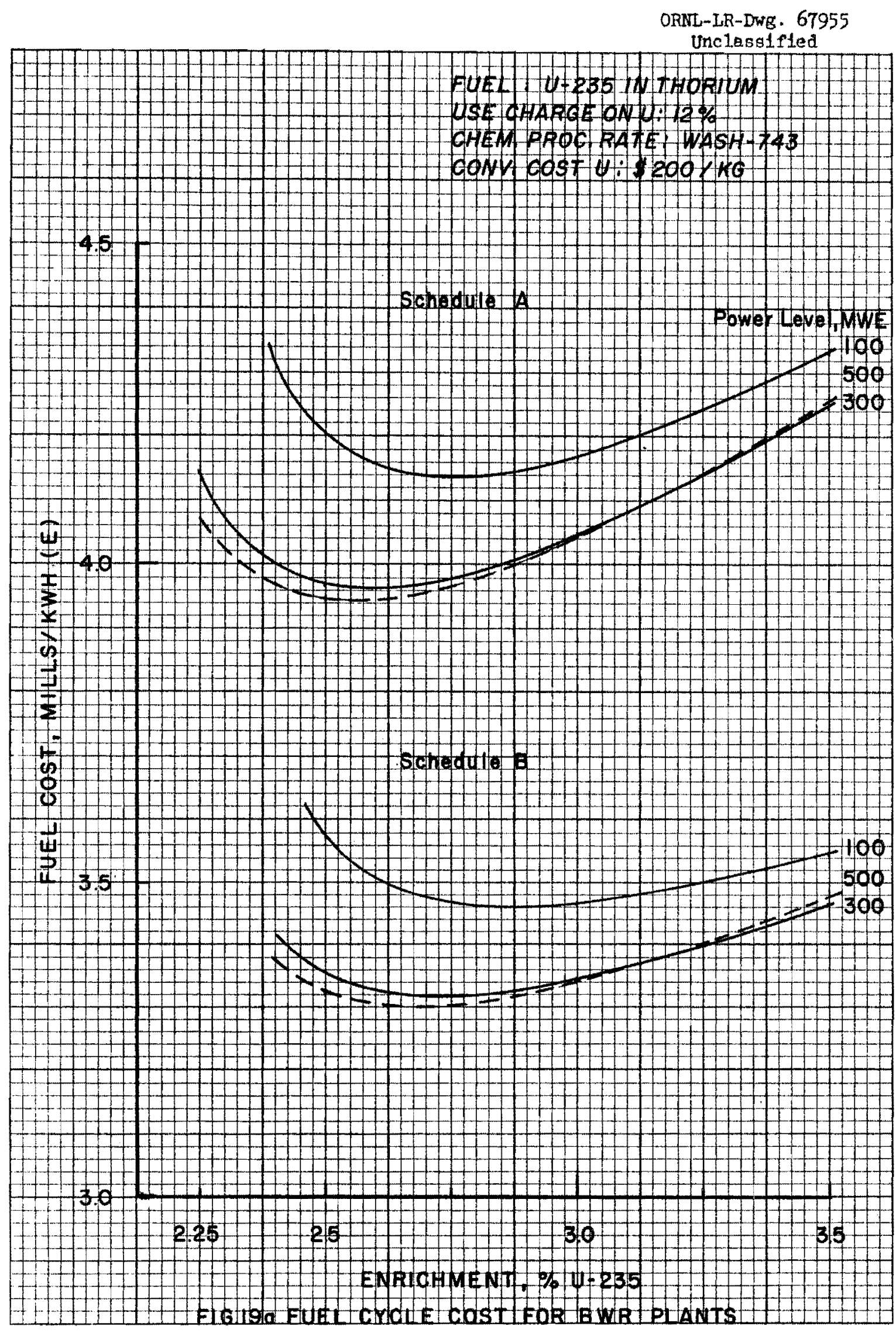


ORNL-LR-DWG . 67956

Unclassified

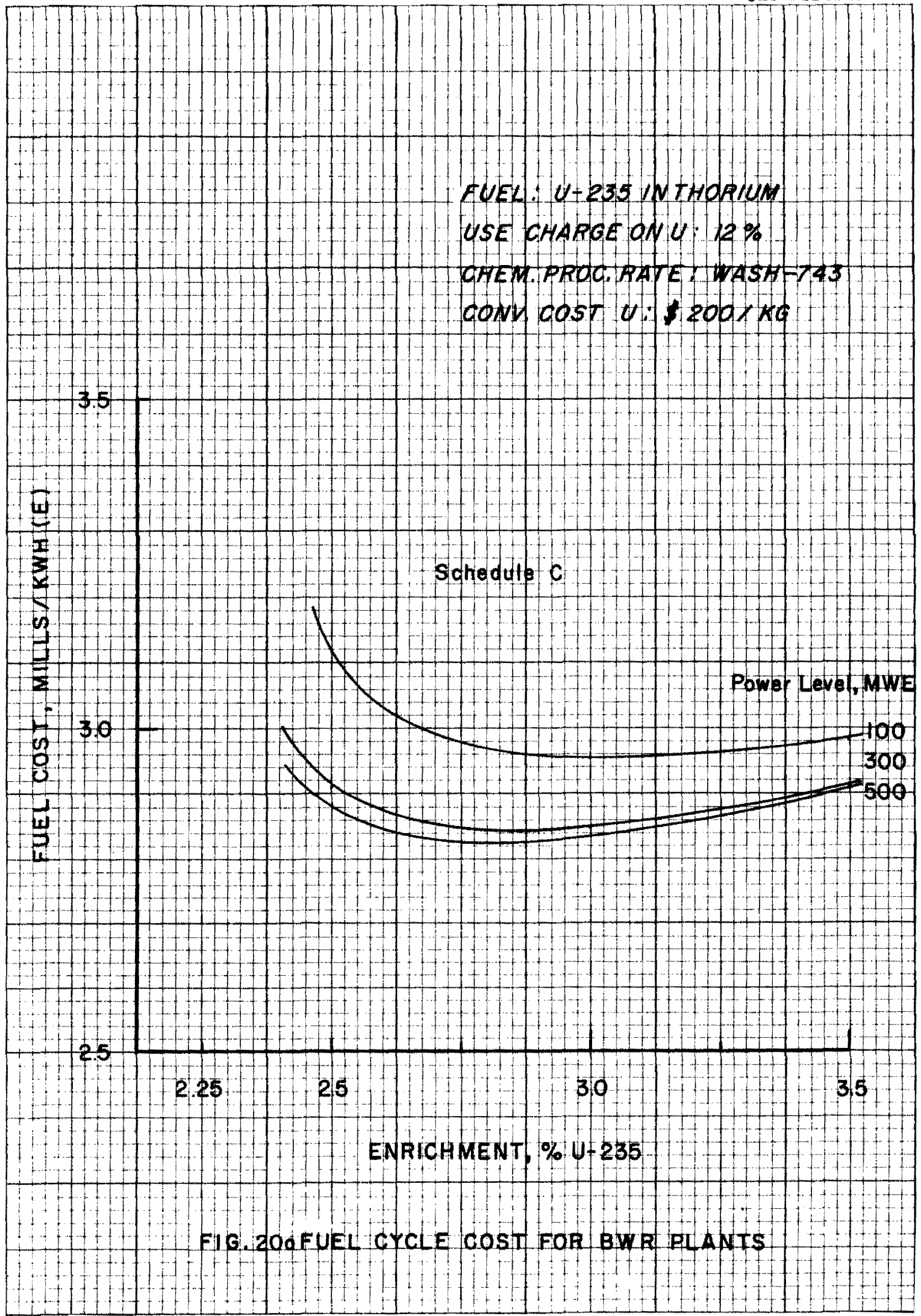


$-45-$

APPENDIX B

DETAILED RESULTS FOR PRESSURIZED WATER

REACTOR CASES 


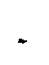

. 
TABLE B-1

FUEL CYCIE COST FOR PRESSURIZED WATER REACTORS

FUEIED WITH ENRICHED URANTUM

Use Charge on Uranium and Plutonium: $4-3 / 4 \%$

\begin{tabular}{|c|c|c|c|c|c|c|c|c|c|}
\hline \multirow[b]{2}{*}{$\begin{array}{l}\text { Cost } \\
\text { Sched- } \\
\text { ule }\end{array}$} & \multirow[b]{2}{*}{$\begin{array}{l}\text { Power } \\
\text { Level, } \\
\text { Mwe }\end{array}$} & \multicolumn{7}{|c|}{ Cost Breakdown, mills/kwhr(e) } & \multirow[b]{2}{*}{$\begin{array}{l}\text { Net } \\
\text { Fue? } \\
\text { Cost }\end{array}$} \\
\hline & & $\begin{array}{l}\text { Fabri- } \\
\text { cation }\end{array}$ & Shipping & Burnup & $\begin{array}{l}\text { Chem. } \\
\text { Proc. }\end{array}$ & $\begin{array}{l}\text { Inven- } \\
\text { tory }\end{array}$ & Interest & $\begin{array}{c}\text { Pu } \\
\text { Credit }\end{array}$ & \\
\hline \multirow[t]{3}{*}{ A } & 100 & .537 & .096 & 1.708 & .157 & .5117 & .260 & .440 & 2.835 \\
\hline & 300 & .541 & .098 & 1.617 & .133 & .450 & .255 & .440 & 2.654 \\
\hline & 500 & .525 & .096 & 1.596 & .125 & .448 & .248 & .434 & 2.610 \\
\hline \multirow[t]{3}{*}{ B } & 100 & .531 & .096 & 1.260 & .153 & .376 & .251 & .330 & 2.337 \\
\hline & 300 & .479 & .087 & 1.207 & .117 & .361 & .248 & .314 & $2.1,85$ \\
\hline & 500 & .466 & .084 & 1.193 & .110 & .360 & .247 & .31 .1 & 2.149 \\
\hline \multirow[t]{3}{*}{ C } & 100 & .445 & .079 & 1.010 & .129 & .357 & .250 & .232 & 2.038 \\
\hline & 300 & .475 & .087 & .941 & .116 & .288 & .243 & .236 & 1.914 \\
\hline & 500 & .463 & .084 & .931 & .109 & .287 & .242 & .233 & 1.883 \\
\hline \multirow[t]{3}{*}{ D } & 100 & .531 & .096 & 1.260 & .154 & .379 & .251 & .440 & 2.237 \\
\hline & 300 & .479 & .087 & 1.207 & .118 & .364 & .248 & .419 & 2.081 \\
\hline & 500 & .466 & .084 & 1.193 & .110 & .364 & .247 & .414 & 2.053 \\
\hline \multirow[t]{3}{*}{$\Phi$} & 100 & .445 & .079 & 1.010 & .131 & .362 & .250 & .413 & 1.864 \\
\hline & 300 & .475 & .087 & .941 & .1118 & .293 & .243 & .419 & 1.738 \\
\hline & 500 & .463 & .084 & .931 & .110 & .293 & .242 & .414 & 1.709 \\
\hline
\end{tabular}


TABLE B-2

FUEL CYCLE COST FOR PRESSURIZED WATER REACTORS

FUELED WITH ENRICHED URANIUM

Use Charge on Uranium and Plutonium: 12\%

\begin{tabular}{|c|c|c|c|c|c|c|c|c|c|}
\hline \multirow[b]{2}{*}{$\begin{array}{l}\text { Cost } \\
\text { Sched- } \\
\text { ule }\end{array}$} & \multirow[b]{2}{*}{$\begin{array}{l}\text { Power } \\
\text { Level, } \\
\text { Mwe }\end{array}$} & \multicolumn{7}{|c|}{ Cost Breakdown, mills/kwhr(e) } & \multirow[b]{2}{*}{$\begin{array}{l}\text { Net } \\
\text { FueI } \\
\text { Cost } \\
\end{array}$} \\
\hline & & $\begin{array}{l}\text { Fabri- } \\
\text { cation }\end{array}$ & Shipping & Burmup & $\begin{array}{l}\text { Chem. } \\
\text { Proc. }\end{array}$ & $\begin{array}{l}\text { Inven- } \\
\text { tory }\end{array}$ & Interest & $\begin{array}{c}\text { Pu } \\
\text { Credit } \\
\end{array}$ & \\
\hline \multirow[t]{3}{*}{ A } & 100 & .696 & .129 & 1.673 & .200 & 1.076 & .285 & .488 & 3.571 \\
\hline & 300 & .724 & .136 & 1.580 & .174 & .916 & .276 & .495 & 3.311 \\
\hline & 500 & .704 & .132 & 1.555 & .163 & .912 & .275 & .492 & 3.249 \\
\hline \multirow[t]{3}{*}{ B } & 100 & .596 & .110 & 1.244 & .171 & .863 & .273 & .346 & 2.911 \\
\hline & 300 & .612 & .1 .14 & 1.174 & .147 & .742 & .266 & .349 & 2.706 \\
\hline & 500 & .592 & .110 & 1.157 & .137 & .738 & .264 & .344 & 2.654 \\
\hline \multirow[t]{3}{*}{$\mathrm{C}$} & 100 & .593 & .110 & .971 & .169 & .690 & .264 & .259 & 2.538 \\
\hline & 300 & .532 & .098 & .930 & .128 & .661 & .260 & .247 & 2.362 \\
\hline & 500 & .516 & .110 & .918 & .120 & .658 & .259 & .244 & 2.323 \\
\hline \multirow[t]{3}{*}{ D } & 100 & .596 & .110 & 1.244 & .172 & .870 & .273 & .461 & 2.804 \\
\hline & 300 & .612 & .114 & 1.174 & .148 & .751 & .266 & .465 & 2.600 \\
\hline & 500 & .592 & .110 & 1.157 & .138 & .748 & .264 & .458 & 2.551 \\
\hline \multirow[t]{3}{*}{$\Sigma$} & 100 & .593 & .110 & .971 & .171 & .702 & .264 & .461 & 2.350 \\
\hline & 300 & .532 & .098 & .930 & .130 & .675 & .260 & .440 & 2.185 \\
\hline & 500 & .516 & .096 & .918 & .122 & .674 & .259 & .434 & 2.151 \\
\hline
\end{tabular}


TABLE B-3

FUEL CYCIE COST FOR PRESSURIZED WATER REACTORS

FUEIED WITH U-235 IN THORIUM

Use Charge on Uranium: $4-3 / 4 \%$

\begin{tabular}{|c|c|c|c|c|c|c|c|c|}
\hline \multirow[b]{2}{*}{$\begin{array}{l}\text { Cost } \\
\text { Sched- } \\
\text { ule }\end{array}$} & \multirow[b]{2}{*}{$\begin{array}{l}\text { Power } \\
\text { Level, } \\
\text { Mwe }\end{array}$} & \multicolumn{7}{|c|}{ Cost Breakdown, mills/kwhr(e) } \\
\hline & & $\begin{array}{l}\text { Fabri- } \\
\text { cation }\end{array}$ & Shipping & $\begin{array}{l}\text { Total } \\
\text { Burnup } \\
\text { Cost }\end{array}$ & $\begin{array}{l}\text { Chem. } \\
\text { Proc. }\end{array}$ & $\begin{array}{l}\text { Inven- } \\
\text { tory }\end{array}$ & Interest & $\begin{array}{l}\text { Net } \\
\text { Fue } 1 \\
\text { Cost } \\
\end{array}$ \\
\hline \multirow[t]{3}{*}{ A } & 100 & .432 & .083 & .833 & .163 & .912 & .261 & 2.684 \\
\hline & 300 & .435 & .085 & .721 & .148 & .851 & .255 & 2.495 \\
\hline & 500 & .415 & .081 & .710 & .137 & .854 & .254 & 2.451 \\
\hline \multirow[t]{3}{*}{ B } & 100 & $\cdot 395$ & .077 & .658 & .147 & .716 & .251 & 2.244 \\
\hline & 300 & .395 & .078 & .578 & .131 & .670 & .246 & 2.098 \\
\hline & 500 & .379 & .074 & .570 & .122 & .672 & .245 & 2.062 \\
\hline \multirow[t]{3}{*}{$\mathrm{C}$} & 100 & .346 & .067 & .528 & .126 & .592 & .245 & 1.904 \\
\hline & 300 & .342 & .067 & .473 & .111 & .556 & .240 & 1.789 \\
\hline & 500 & .350 & .069 & .447 & .111 & .546 & .238 & 1.761 \\
\hline \multirow[t]{3}{*}{ D } & 100 & .425 & .083 & .404 & .161 & .745 & .250 & 2.068 \\
\hline & 300 & .430 & .085 & .314 & .146 & .703 & .246 & 1.924 \\
\hline & 500 & .410 & .081 & .313 & .135 & .708 & .244 & 1.891 \\
\hline \multirow[t]{3}{*}{$E$} & 100 & .421 & .083 & .085 & .160 & .632 & .243 & 1.624 \\
\hline & 300 & .425 & .085 & .011 & .145 & .601 & .239 & 1.506 \\
\hline & 500 & .446 & .090 & -.035 & .148 & .592 & .238 & 1.479 \\
\hline
\end{tabular}


TABLE B-4

FUEL CYCLE COST FOR PRESSURIZED WATER REACTORS

FUELED WITH U-235 IN THORIUM

Use Charge on Uranium: $12 \%$

\begin{tabular}{|c|c|c|c|c|c|c|c|c|}
\hline \multirow[b]{2}{*}{$\begin{array}{l}\text { Cost } \\
\text { Sched- } \\
\text { ule }\end{array}$} & \multirow[b]{2}{*}{$\begin{array}{l}\text { Power } \\
\text { Level, } \\
\text { Mwe }\end{array}$} & \multicolumn{7}{|c|}{ Cost Breakdown, mills/kwhr(e) } \\
\hline & & $\begin{array}{l}\text { Fabri- } \\
\text { cation }\end{array}$ & Shipping & $\begin{array}{l}\text { Total } \\
\text { Burnup } \\
\text { Cost }\end{array}$ & $\begin{array}{l}\text { Chem. } \\
\text { Proc. }\end{array}$ & $\begin{array}{l}\text { Inven- } \\
\text { tory }\end{array}$ & Interest & $\begin{array}{l}\text { Net } \\
\text { Euel } \\
\text { Cost } \\
\end{array}$ \\
\hline \multirow[t]{3}{*}{ A } & 100 & .470 & .091 & .797 & .179 & 2.131 & .304 & 3.972 \\
\hline & 300 & .480 & .094 & .678 & .164 & 1.979 & .293 & 3.688 \\
\hline & 500 & .455 & .090 & .667 & .151 & 1.987 & .292 & 3.642 \\
\hline \multirow[t]{3}{*}{ B } & 100 & .463 & .091 & .607 & .174 & 1.610 & .282 & 3.227 \\
\hline & 300 & .474 & .094 & .518 & .159 & 1.497 & .274 & 3.016 \\
\hline & 500 & .450 & .090 & .509 & .147 & 1.503 & .273 & 2.972 \\
\hline \multirow[t]{3}{*}{$\mathrm{C}$} & 100 & .391 & .077 & .493 & .144 & 1.313 & .270 & 2.688 \\
\hline & 300 & .391 & .078 & .432 & .128 & 1.221 & .263 & 2.513 \\
\hline & 500 & .375 & .074 & .426 & .120 & 1.226 & .262 & 2.483 \\
\hline \multirow[t]{3}{*}{$\mathrm{D}$} & 100 & .463 & .091 & .364 & .177 & 1.721 & .282 & 3.098 \\
\hline & 300 & .474 & .094 & .265 & .162 & 1.614 & .274 & 2.883 \\
\hline & 500 & .450 & .090 & .265 & .149 & 1.627 & .273 & 2.854 \\
\hline \multirow[t]{3}{*}{$\mathrm{E}$} & 100 & .509 & .102 & -.012 & .195 & 1.407 & .267 & 2.468 \\
\hline & 300 & .470 & .094 & -.043 & .161 & 1.348 & .261 & 2.291 \\
\hline & 500 & .446 & .090 & -.035 & .148 & 1.366 & .260 & 2.275 \\
\hline
\end{tabular}


TABIE B-5

COST PARAMETERS OF OPTIMUM CASES FOR PRESSURIZED

WATER REACTORS FUETIED WITH ENRICHED URANIUM

Use Charge on Uranium and Plutonium: $4-3 / 4 \%$

\begin{tabular}{|c|c|c|c|c|c|c|c|}
\hline $\begin{array}{l}\text { Cost } \\
\text { Sched- } \\
\text { ule }\end{array}$ & $\begin{array}{l}\text { Power } \\
\text { Leve I, } \\
\text { Mwe }\end{array}$ & $\begin{array}{l}\text { Initial } \\
\text { wt. \% } \\
\text { U-235 }\end{array}$ & $\begin{array}{l}\text { Fina. } \\
\text { wt. \% } \\
\text { U-235 }\end{array}$ & $\begin{array}{l}\text { Final Pu } \\
\mathrm{gm} / \mathrm{kgU}, \\
\text { final }\end{array}$ & $\begin{array}{l}\text { Exposure } \\
\text { Mwd } / M^{m}\end{array}$ & $\begin{array}{l}\text { Lifetime, } \\
\text { days at } \\
\text { full power }\end{array}$ & $\frac{\mathrm{kg} \text { final }}{\mathrm{kgU} \text { initial }}$ \\
\hline \multirow[t]{3}{*}{ A } & 100 & 5.0 & 2.003 & 12.32 & 33,270 & 1700 & .9518 \\
\hline & 300 & 4.5 & 1.680 & 12.62 & 32,490 & 1660 & .9529 \\
\hline & 500 & 4.5 & 1.626 & 12.84 & 33,470 & 1710 & .9517 \\
\hline \multirow[t]{3}{*}{ B } & 100 & 5.0 & 2.003 & 12.32 & 33,270 & 1700 & .9518 \\
\hline & 300 & 5.0 & 1.791 & 13.70 & 36,890 & 1885 & .9470 \\
\hline & 500 & 5.0 & 1.738 & 13.91 & 37,870 & 1935 & .9457 \\
\hline \multirow[t]{3}{*}{ C } & 100 & 6.0 & 2.302 & 14.94 & 40,610 & 2075 & .9418 \\
\hline & 300 & 5.0 & 1.791 & 13.70 & 36,890 & 1885 & .9470 \\
\hline & 500 & 5.0 & 1.738 & $1.3 \cdot 91$ & 37,870 & 1935 & .9457 \\
\hline \multirow[t]{3}{*}{$D$} & 100 & 5.0 & 2.003 & 1.2 .32 & 33,270 & 1700 & .9518 \\
\hline & 300 & 5.0 & 1.791 & $13 \cdot 70$ & 36,890 & 1885 & .9470 \\
\hline & 500 & 5.0 & 1.738 & 13.91 & 37,870 & 1.935 & .9457 \\
\hline \multirow[t]{3}{*}{$\mathrm{E}$} & 100 & 6.0 & 2.302 & 1.4 .94 & 40,610 & 2085 & .9419 \\
\hline & 300 & 5.0 & 1.791 & 13.70 & 36,890 & 1885 & .9470 \\
\hline & 500 & 5.0 & 1.738 & $13 \cdot 91$ & 37,870 & 1935 & .9457 \\
\hline
\end{tabular}




\section{TABIE B-6}

\section{COST PARAMETERS OF OPTIMUM CASES FOR PRESSURIZED}

WATER REACTORS FUEIED WITH ENRICHED URANIUM

Use Charge on Uranium and Plutonium: $12 \%$

\begin{tabular}{|c|c|c|c|c|c|c|c|}
\hline $\begin{array}{l}\text { Cost } \\
\text { Sched- } \\
\text { ule }\end{array}$ & $\begin{array}{l}\text { Power } \\
\text { Leve1, } \\
\text { Mwe }\end{array}$ & $\begin{array}{l}\text { Initial } \\
\text { wt. \% } \\
\mathrm{U}-235\end{array}$ & $\begin{array}{l}\text { Final } \\
\text { wt. \% } \\
\mathrm{U}-235 \\
\end{array}$ & $\begin{array}{l}\text { Final Pu } \\
\mathrm{gm} / \mathrm{kgU} \\
\text { final }\end{array}$ & $\begin{array}{l}\text { Exposure } \\
\text { Mwd/MT }\end{array}$ & $\begin{array}{l}\text { Iifetime, } \\
\text { days at } \\
\text { full power }\end{array}$ & $\frac{\mathrm{kgU} \text { final }}{\mathrm{kgU} \text { initial }}$ \\
\hline \multirow[t]{3}{*}{ A } & 100 & 4.0 & 1.757 & 10.55 & 24,850 & 1270 & .9632 \\
\hline & 300 & $3 \cdot 5$ & 1.470 & 10.15 & 23,300 & 1195 & .9651 \\
\hline & 500 & 3.5 & 1.419 & 10.32 & 24,170 & 1235 & .9641 \\
\hline \multirow[t]{3}{*}{ B } & 100 & 4.5 & 1.876 & 11.76 & 29,160 & 1490 & .9574 \\
\hline & 300 & 4.0 & 1.571 & 11.38 & 28,000 & 1430 & .9590 \\
\hline & 500 & 4.0 & 1.516 & 11.61 & 28,960 & 1480 & .9577 \\
\hline \multirow[t]{3}{*}{$\mathrm{C}$} & 100 & 4.5 & 1.876 & 11.76 & 29,160 & 1490 & $.957^{4}$ \\
\hline & 300 & 4.5 & 1.680 & 12.62 & 32,490 & 1660 & .9529 \\
\hline & 500 & 4.5 & 1.626 & 12.84 & 33,470 & 1710 & .9517 \\
\hline \multirow[t]{3}{*}{$D$} & 100 & 4.5 & 1.876 & 11.76 & 29,160 & 1490 & $.957^{4}$ \\
\hline & 300 & 4.0 & 1.571 & 11.38 & 28,000 & 1430 & .9590 \\
\hline & 500 & 4.0 & 1.516 & 11.61 & 28,960 & 1480 & .9577 \\
\hline \multirow[t]{3}{*}{ E } & 100 & 4.5 & 1.876 & 11.76 & 29,160 & 1490 & .9574 \\
\hline & 300 & 4.5 & 1.680 & 12.62 & 32,490 & 1660 & .9529 \\
\hline & 500 & 4.5 & 1.626 & 12.84 & 33,470 & 1710 & .9517 \\
\hline
\end{tabular}


TABLE B-7

COST PARAMETERS OF NEAR-OPTIMUM CASES FOR PRESSURIZED

WATER REACTORS FUELED WITH U-235 IN THORIUM

Use Charge on Uranium: $4-3 / 4 \%$

\begin{tabular}{|c|c|c|c|c|c|c|c|c|}
\hline $\begin{array}{l}\text { Cost } \\
\text { Sched- } \\
\text { ule }\end{array}$ & $\begin{array}{l}\text { Power } \\
\text { Level, } \\
\text { Mwe }\end{array}$ & $\begin{array}{l}\text { Initial } \\
\text { wt. \% } \\
\text { U- } 235 \\
\text { in fuel }\end{array}$ & $\begin{array}{l}\text { Final } \\
\text { wt. \% U } \\
\text { in fuel }\end{array}$ & $\begin{array}{l}\text { wh. \% } \\
\mathrm{U}-235 \\
\text { in Fi- } \\
\text { nal U }\end{array}$ & $\begin{array}{l}\text { wt. } \% \\
\mathrm{U}-233 \\
\text { in Fi- } \\
\text { nal U }\end{array}$ & $\begin{array}{l}\text { Final } \\
k E \text { fuel } \\
\text { initial } \\
k g \text { fuel }\end{array}$ & $\begin{array}{l}\text { Expo- } \\
\text { sure } \\
\text { Mwa/MT }\end{array}$ & $\begin{array}{l}\text { Lifetime, } \\
\text { days at } \\
\text { full } \\
\text { power } \\
\end{array}$ \\
\hline \multirow[t]{3}{*}{ A } & 100 & 4.75 & 4.058 & 38.26 & 40.57 & .9592 & 38,240 & 2095 \\
\hline & 300 & 4.25 & 3.739 & 34.31 & 44.01 & .9602 & 37,300 & 2045 \\
\hline & 500 & 4.25 & 3.722 & 32.50 & 45.04 & .9582 & 39,150 & 21.45 \\
\hline \multirow[t]{3}{*}{ B } & 100 & 5.0 & 3.759 & 37.39 & 40.53 & .9558 & 41,430 & 2270 \\
\hline & 300 & 4.5 & 3.863 & 33.25 & 44.00 & .9563 & 40,900 & 2240 \\
\hline & 500 & 4.5 & 3.846 & 31.66 & 44.89 & .9545 & 42,600 & 2335 \\
\hline \multirow[t]{3}{*}{$\mathrm{C}$} & 100 & 5.5 & 4.435 & 36.18 & 40.19 & .9491 & 47,500 & 2600 \\
\hline & 300 & 5.0 & 4.308 & 32.03 & 43.48 & .9492 & 47.370 & 2595 \\
\hline & 500 & 4.75 & 3.969 & 31.08 & 44.59 & .9508 & 45,910 & 2515 \\
\hline \multirow[t]{3}{*}{ D } & 100 & 4.75 & 4.058 & 38.26 & 40.57 & .9592 & 38,240 & 2095 \\
\hline & 300 & 4.25 & 3.739 & 34.31 & 44.01 & .9602 & 37,300 & 2045 \\
\hline & 500 & 4.25 & 3.722 & 32.50 & 45.04 & .9582 & 39,150 & 2145 \\
\hline \multirow[t]{3}{*}{$\mathrm{E}$} & 100 & 4.75 & 4.058 & 38.26 & 40.57 & .9592 & 38,240 & 2095 \\
\hline & 300 & 4.25 & 3.739 & 34.31 & 44.01 & .9602 & 37,300 & 2045 \\
\hline & 500 & 4.0 & 3.595 & 33.72 & 44.99 & .9623 & 35,400 & 1940 \\
\hline
\end{tabular}


TABLE B-8

COST PARAMETERS OF NEAR-OPTIMUM CASES FOR PRESSURIZED

WATER REACTORS FUEIED WITH U-235 IN THORIUM

Use Charge on Uranium: 12\%

\begin{tabular}{|c|c|c|c|c|c|c|c|c|}
\hline $\begin{array}{l}\text { Cost } \\
\text { Sched- } \\
\text { ule }\end{array}$ & $\begin{array}{l}\text { Power } \\
\text { Level, } \\
\text { Mwe }\end{array}$ & $\begin{array}{l}\text { Initial } \\
\text { wt. \% } \\
\text { U-235 } \\
\text { in fuel }\end{array}$ & $\begin{array}{l}\text { Final } \\
\text { wt. \% U } \\
\text { in fuel }\end{array}$ & $\begin{array}{l}\text { wt. \% } \\
\mathrm{U}-235 \\
\text { in Fi- } \\
\text { nal U }\end{array}$ & $\begin{array}{l}\text { wt. } \% \\
U-233 \\
\text { in Fi- } \\
\text { nal U }\end{array}$ & $\begin{array}{l}\text { Final } \\
\mathrm{kg} \text { fuel } \\
\text { initial } \\
\mathrm{kg} \text { fuel }\end{array}$ & $\begin{array}{l}\text { Expo- } \\
\text { sure } \\
\text { Mwd/MT }\end{array}$ & $\begin{array}{l}\text { Lifetime, } \\
\text { days at } \\
\text { full } \\
\text { power } \\
\end{array}$ \\
\hline \multirow[t]{3}{*}{$A$} & 100 & 4.5 & 3.929 & 39.48 & 40.39 & .9628 & 34,850 & 1910 \\
\hline & 300 & 4.0 & 3.610 & 35.73 & 43.80 & .9643 & 33,600 & 1840 \\
\hline & 500 & 4.0 & 3.595 & 33.72 & 44.99 & .9623 & 35,400 & 1940 \\
\hline \multirow[t]{3}{*}{ B } & 100 & 4.5 & 3.929 & 39.48 & 40.39 & .9628 & 34,850 & 1910 \\
\hline & 300 & 4.0 & 3.610 & 35.73 & 43.80 & .9643 & 33,600 & 1840 \\
\hline & 500 & 4.0 & 3.595 & 33.72 & 44.99 & .9623 & 35,400 & 1940 \\
\hline \multirow[t]{3}{*}{ C } & 100 & 5.0 & 3.759 & $37 \cdot 39$ & 40.53 & .9558 & 41,430 & 2270 \\
\hline & 300 & $4 \cdot 5$ & 3.863 & 33.25 & 44.00 & .9563 & 40,900 & 2240 \\
\hline & 500 & 4.5 & 3.846 & 31.66 & 44.89 & .9545 & 42,600 & 2335 \\
\hline \multirow[t]{3}{*}{$D$} & 100 & $4 \cdot 5$ & 3.929 & 39.48 & 40.39 & .9628 & 34,850 & 1910 \\
\hline & 300 & 4.0 & 3.610 & 35.73 & 43.80 & .9643 & 33,600 & 1840 \\
\hline & 500 & 4.0 & 3.595 & 33.72 & 44.99 & .9623 & 35,400 & 1940 \\
\hline \multirow[t]{3}{*}{$\mathrm{E}$} & 100 & 4.25 & 3.800 & 41.18 & 39.92 & .9667 & 31,210 & 1710 \\
\hline & 300 & 4.0 & 3.610 & 35.73 & 43.80 & .9643 & 33,600 & 1840 \\
\hline & 500 & 4.0 & 3.595 & 33.72 & 44.99 & .9623 & 35,400 & 1940 \\
\hline
\end{tabular}


ORNL-LR-DWE . 67957

Unclassifled

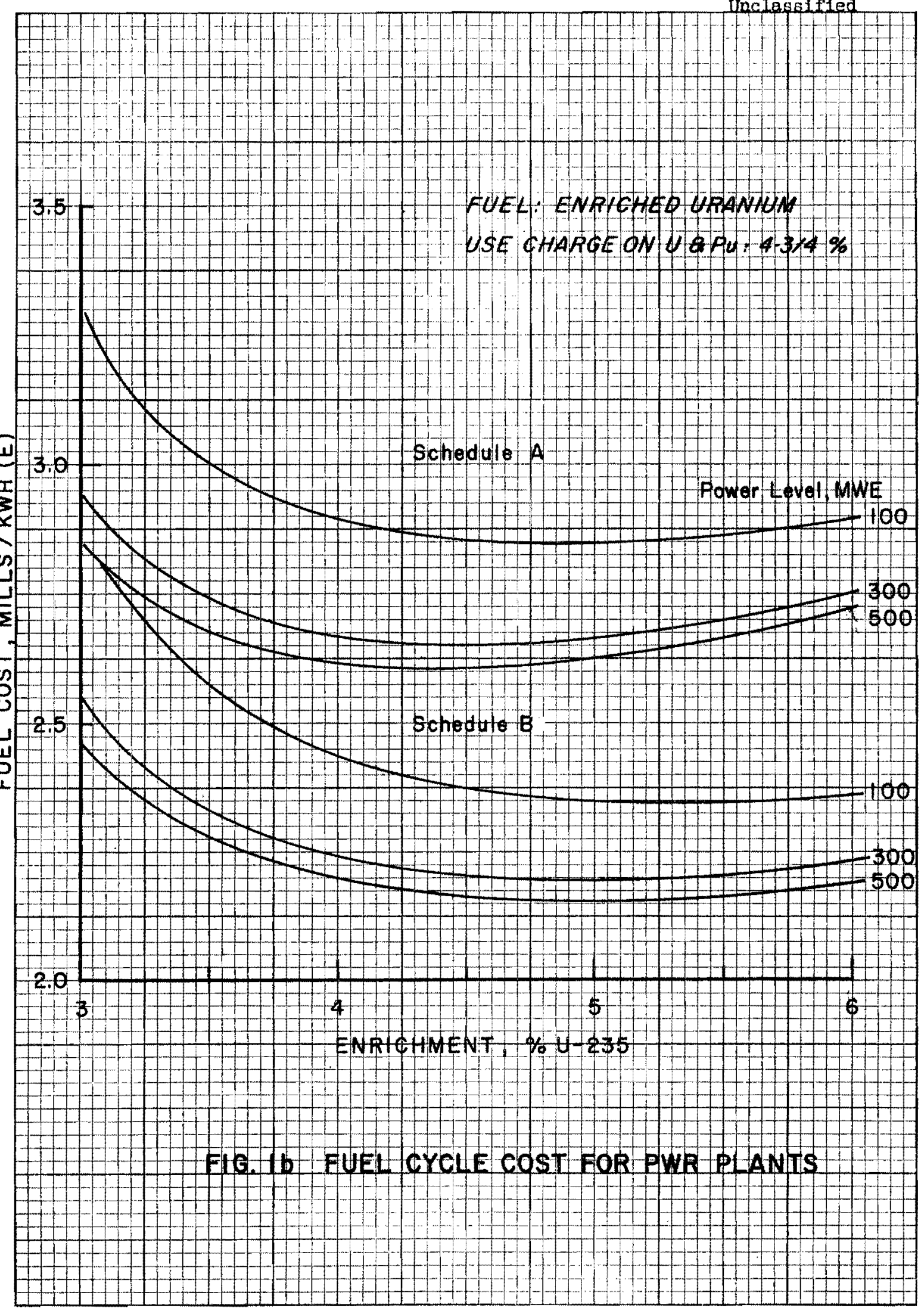


ORNL-LR-DWg . 67958 Unclassified

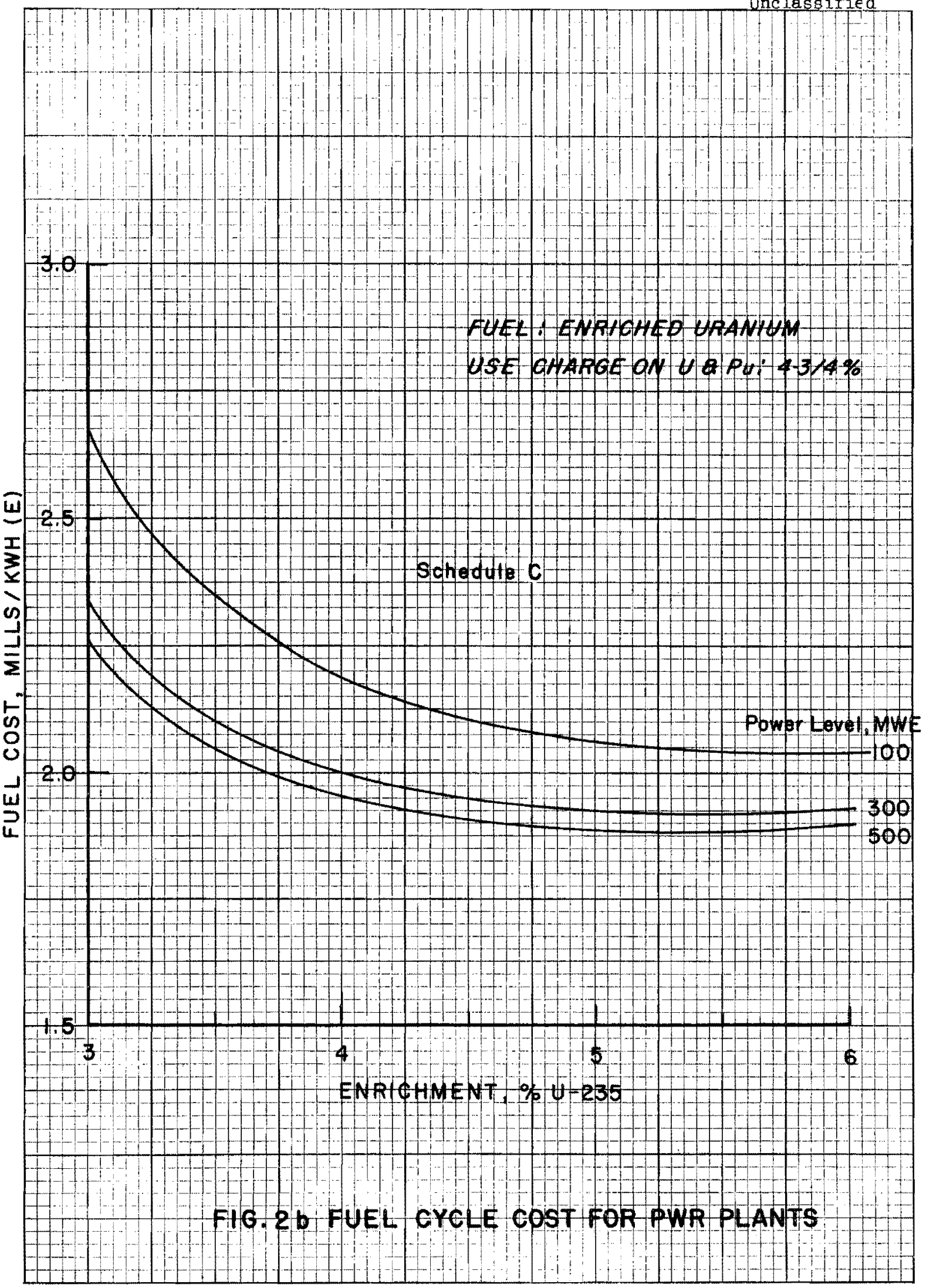


ORNL-LR-DWg . 67959

Unclasstfied

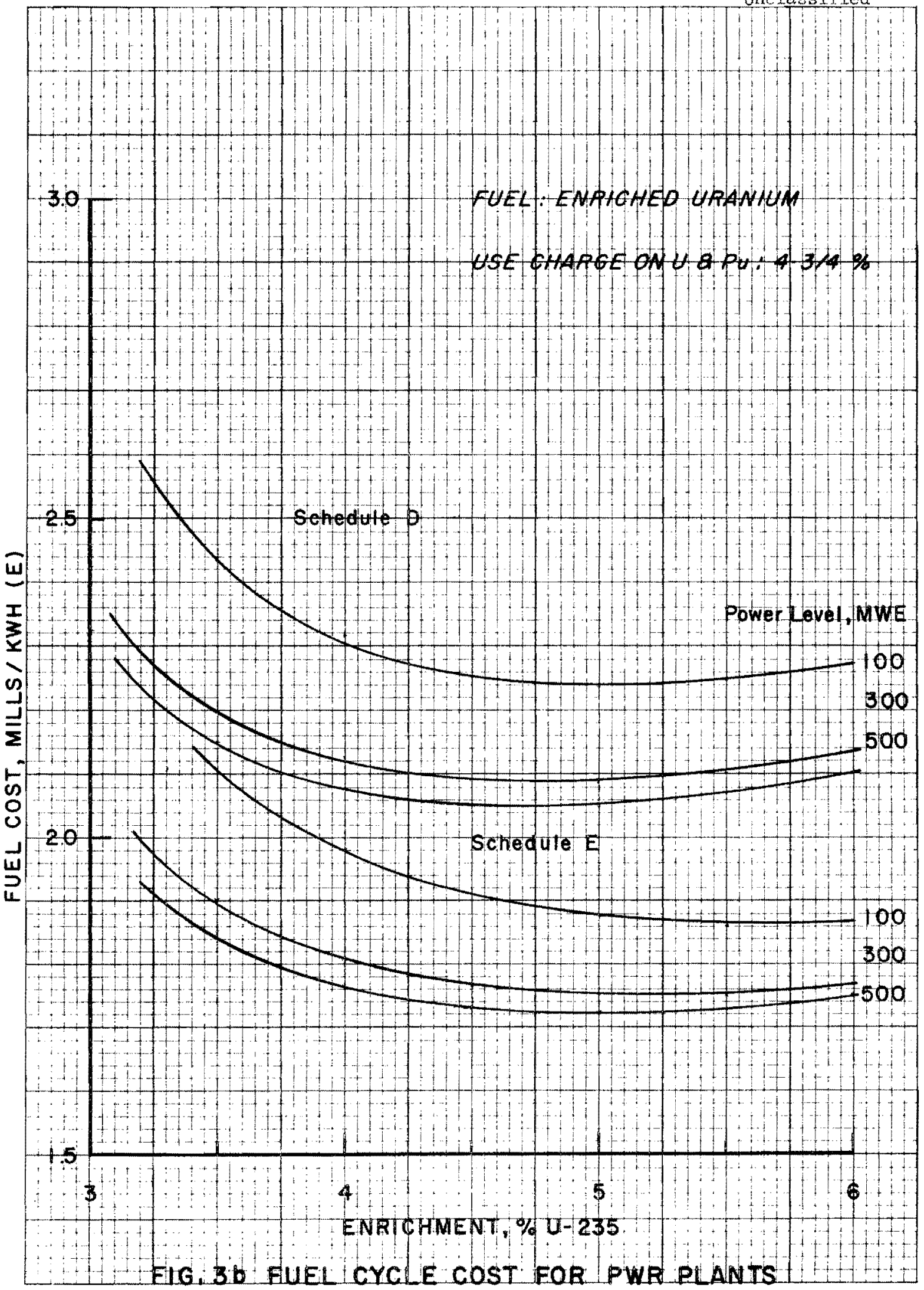




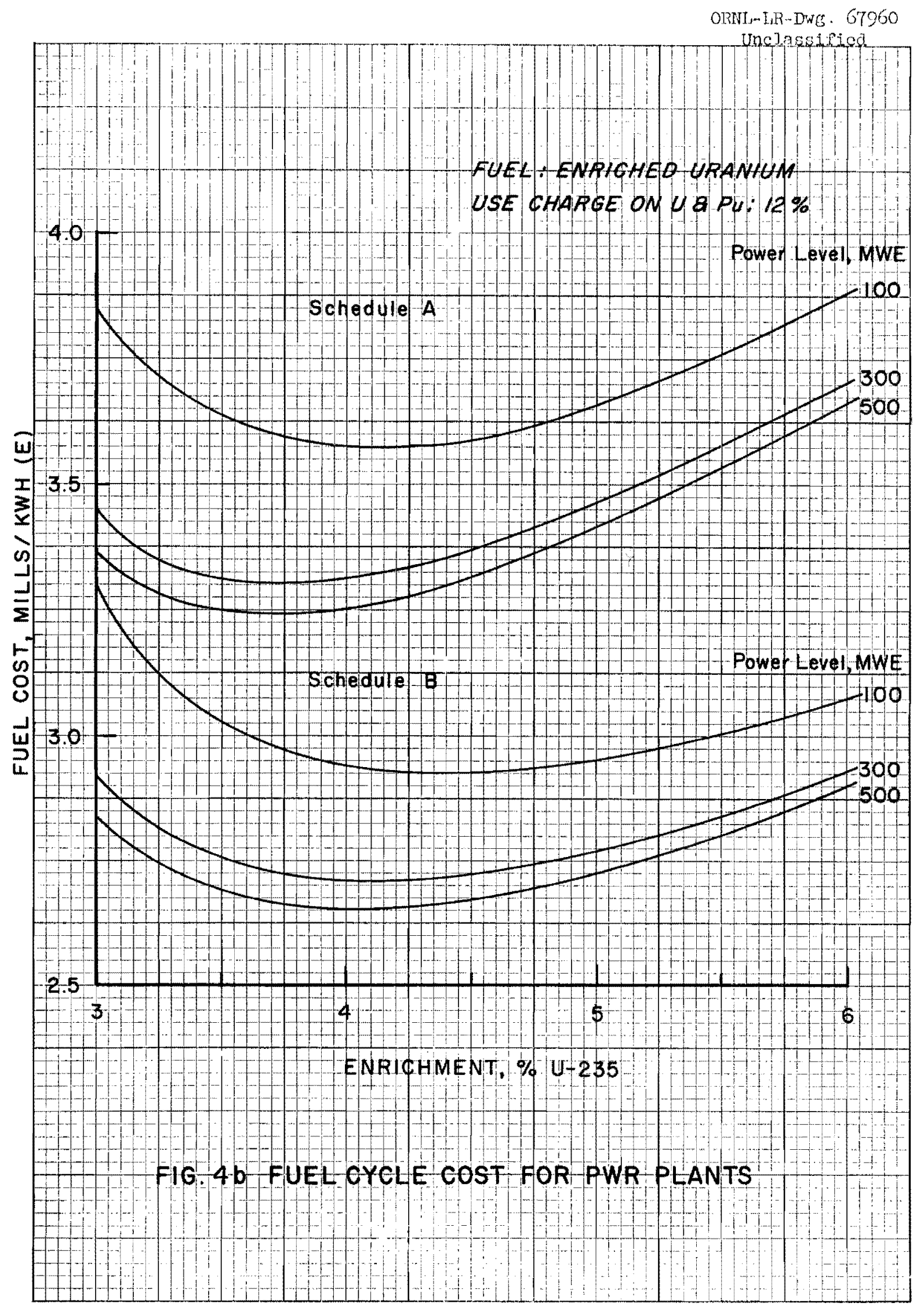


ORNL-LR-DWE. 67961

Unclassfied

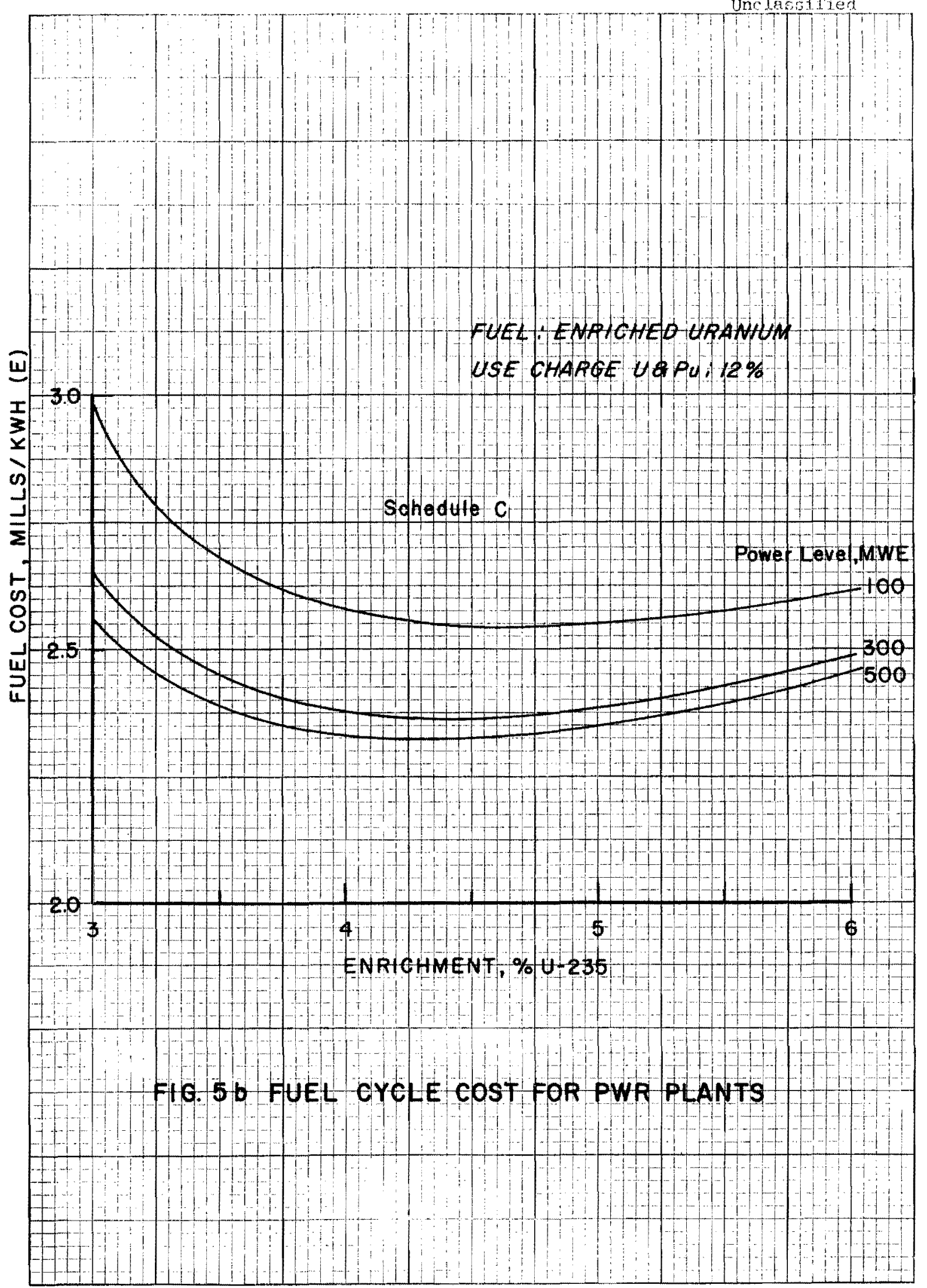


ORNL-LR-DWE . 67962

Unclassified

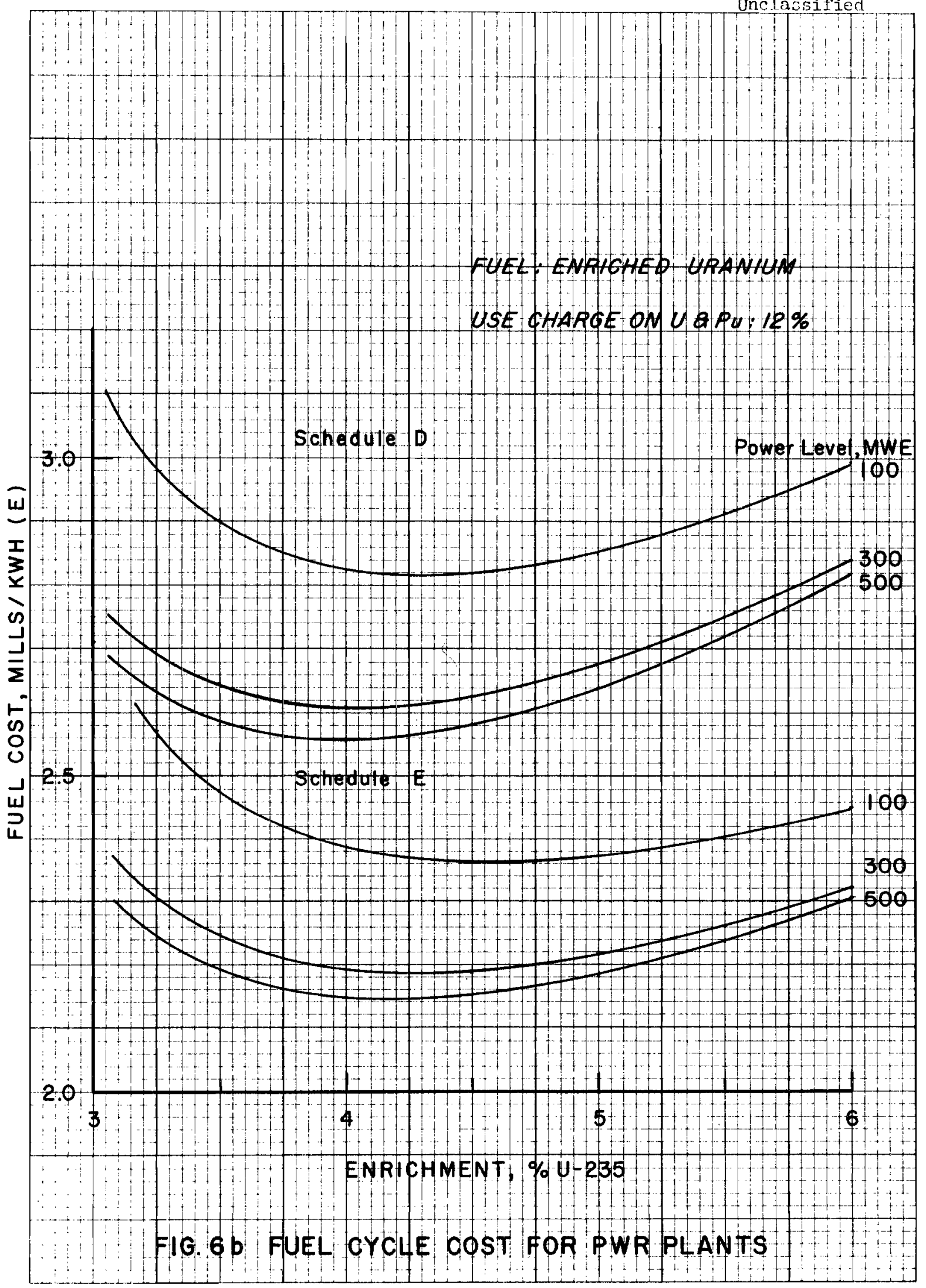


ORNL-LR-Dwg . 67963

Unclassified

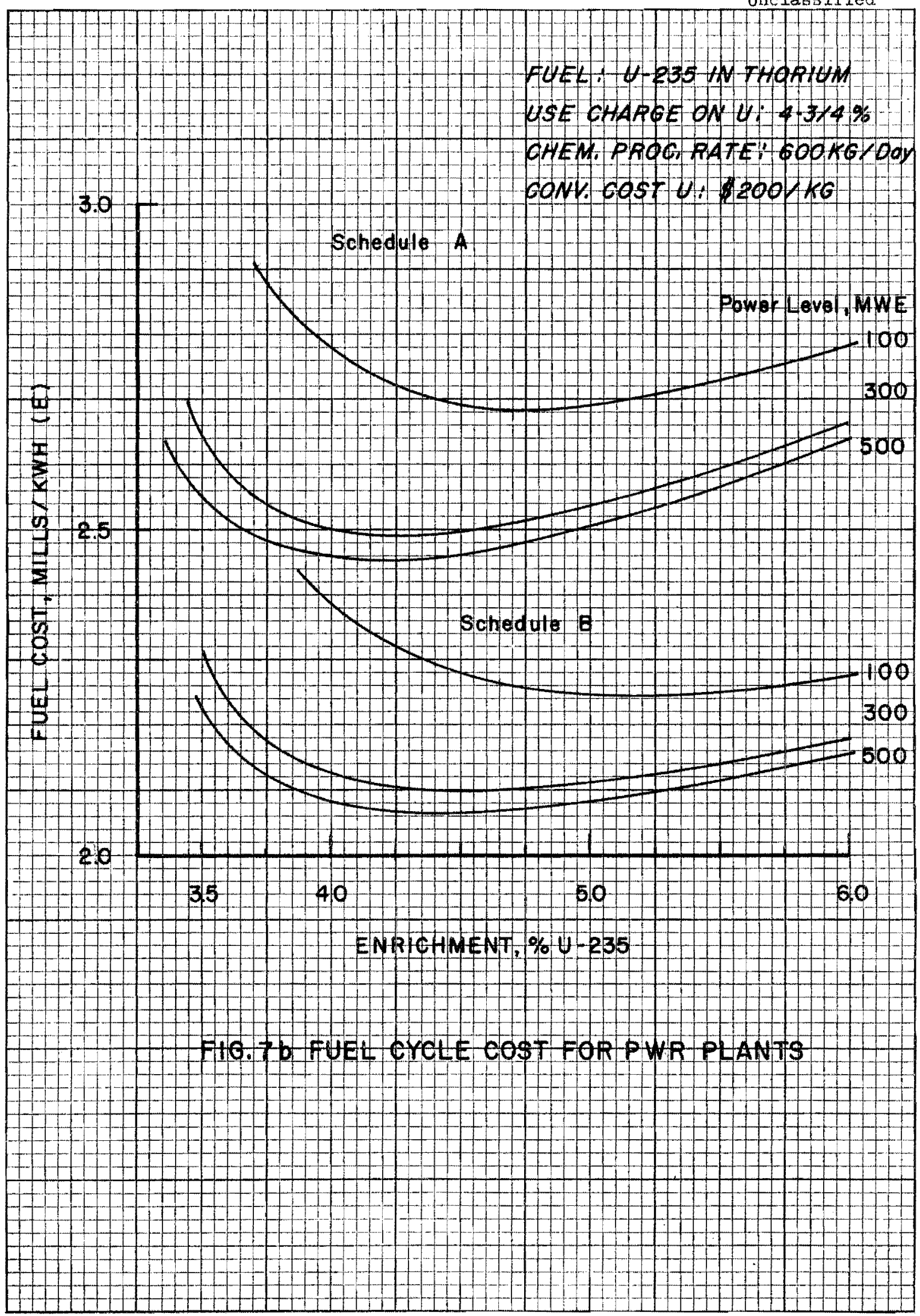


ORNL-LR-Dwg . 67,54 Unclaselfted.

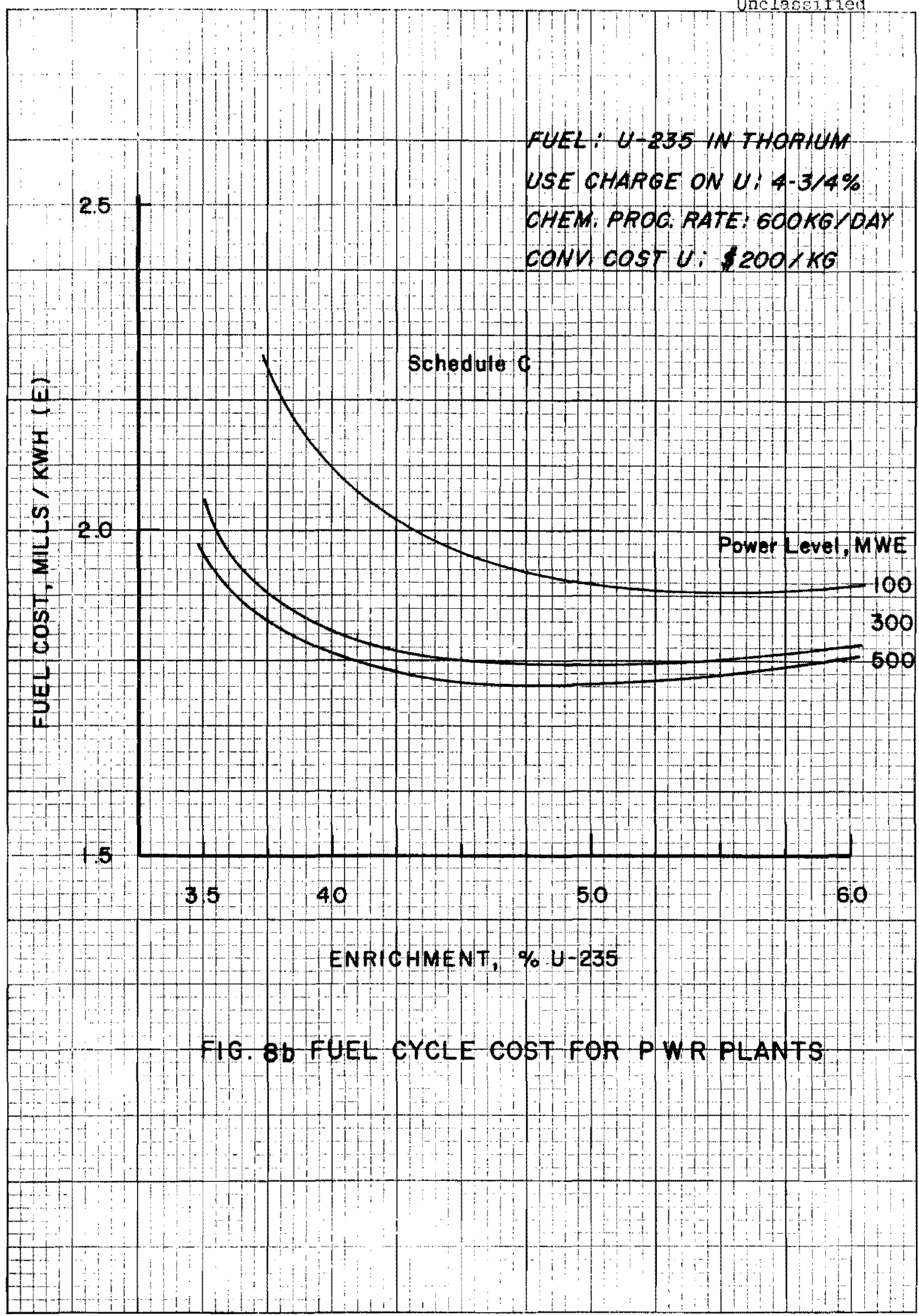


ONM-IR-DWS . 67065

unclazelfied

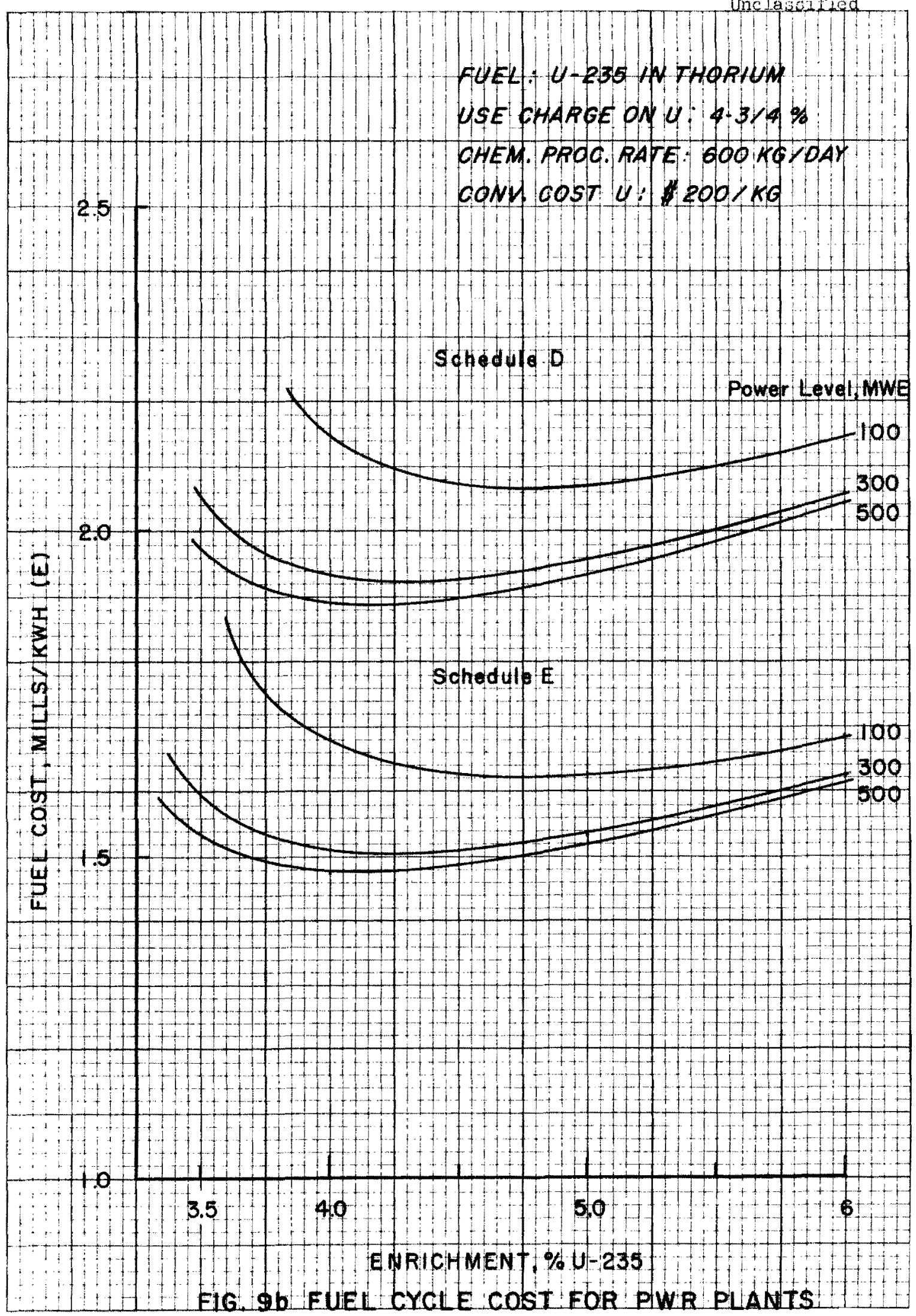


ORNL-LR-DWG. 67966

Unclassified

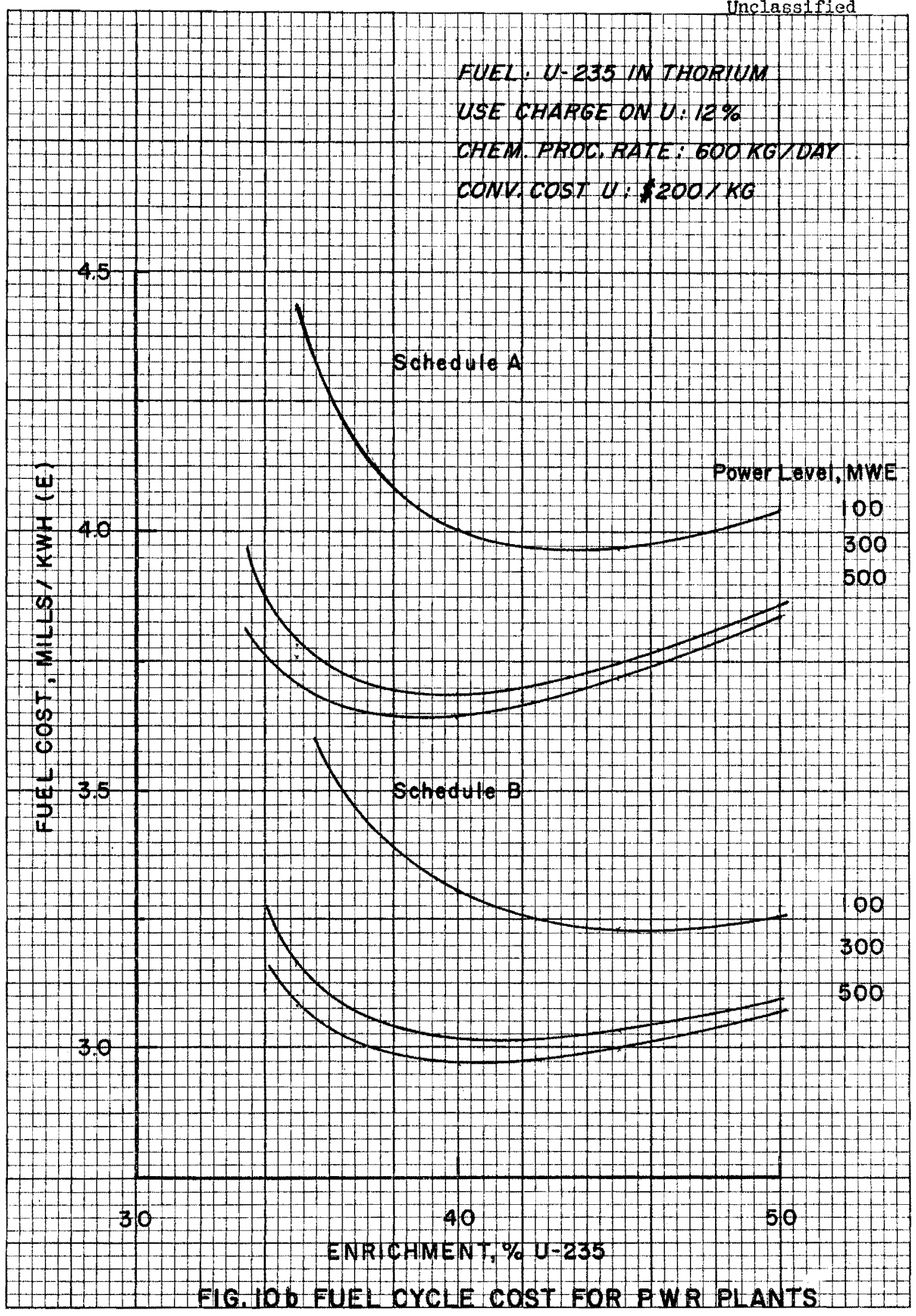




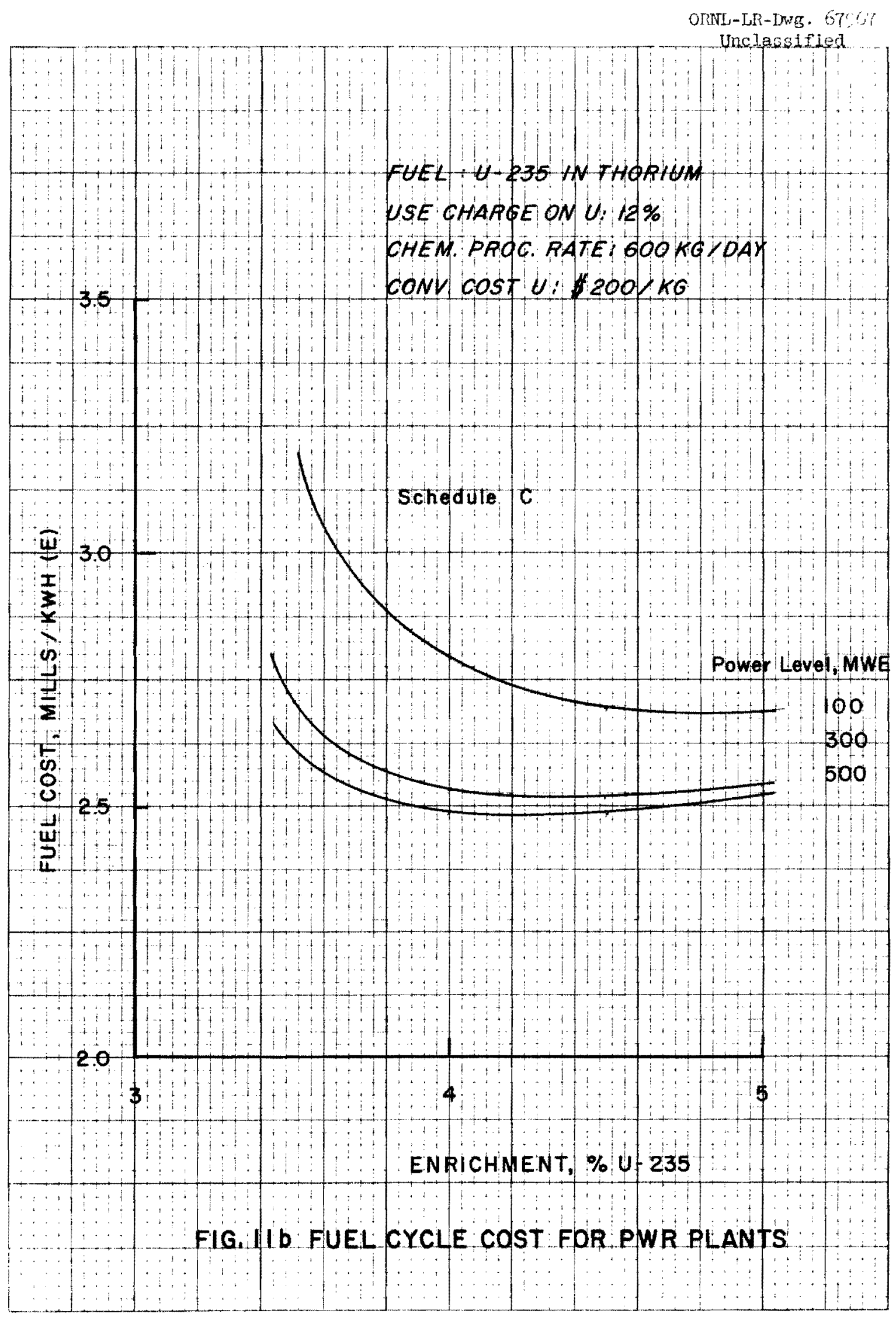


ORNL-LR-DWCS. 67)6?

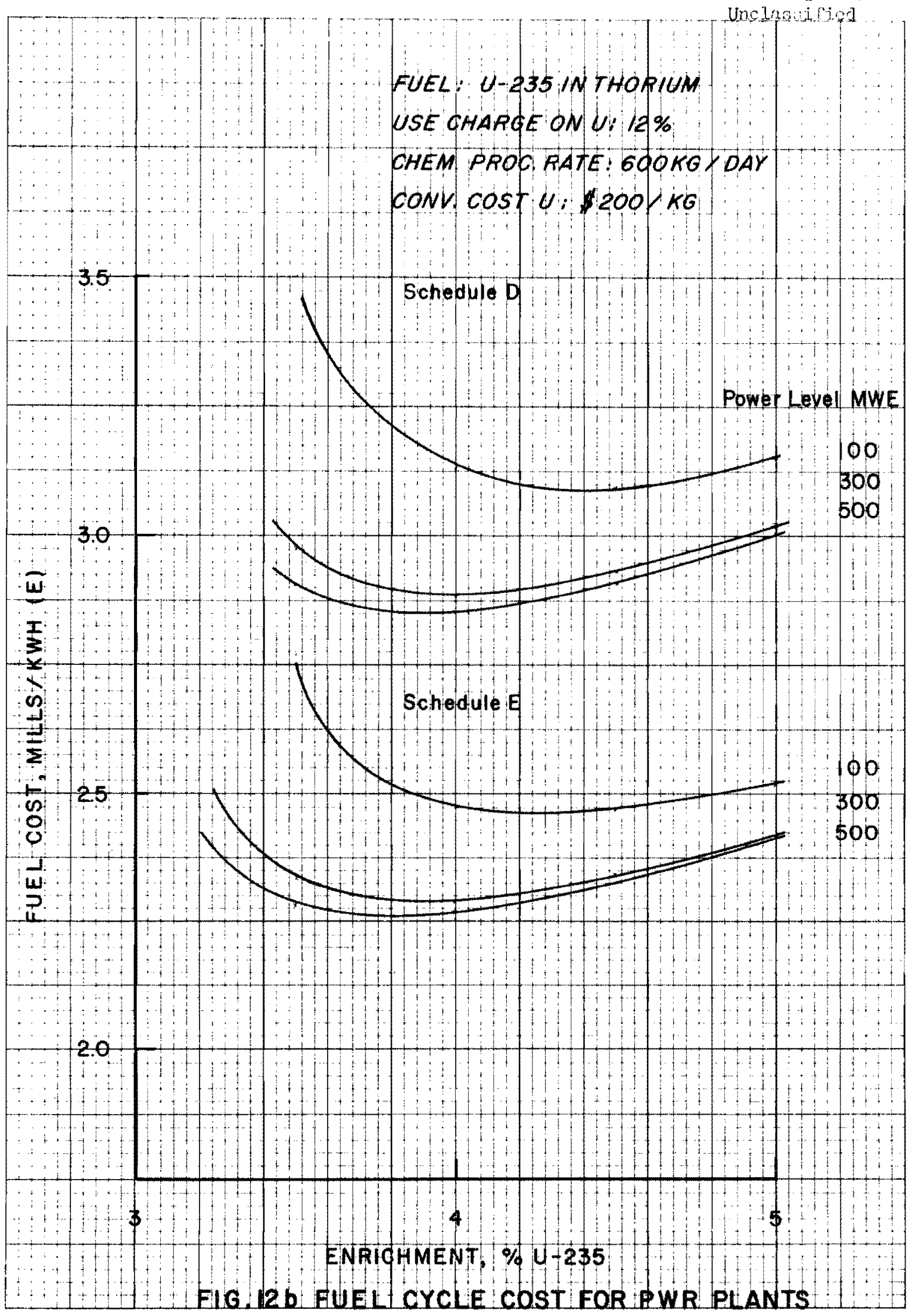


ORNL-LR-DWg . 67969

inclessifted

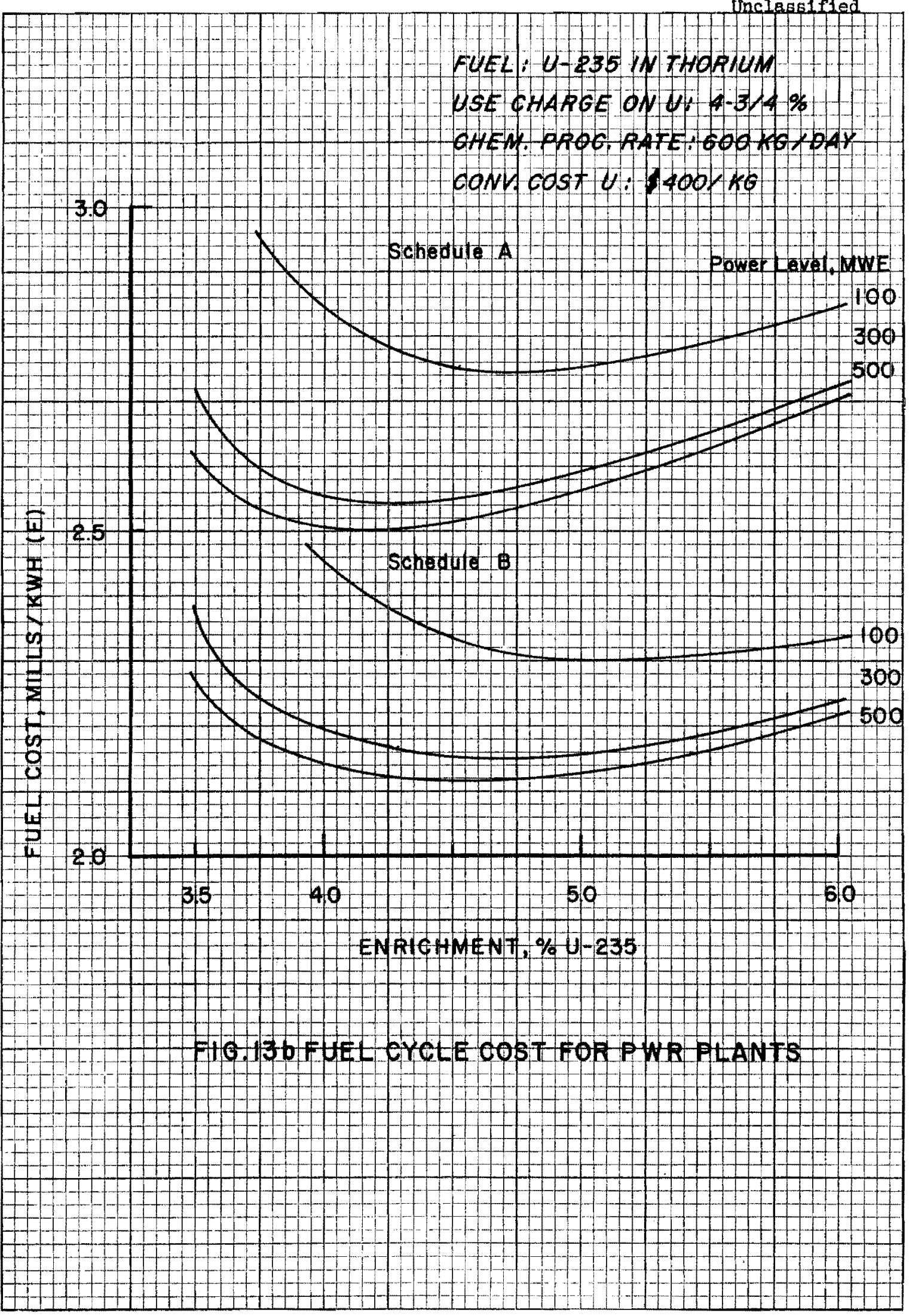




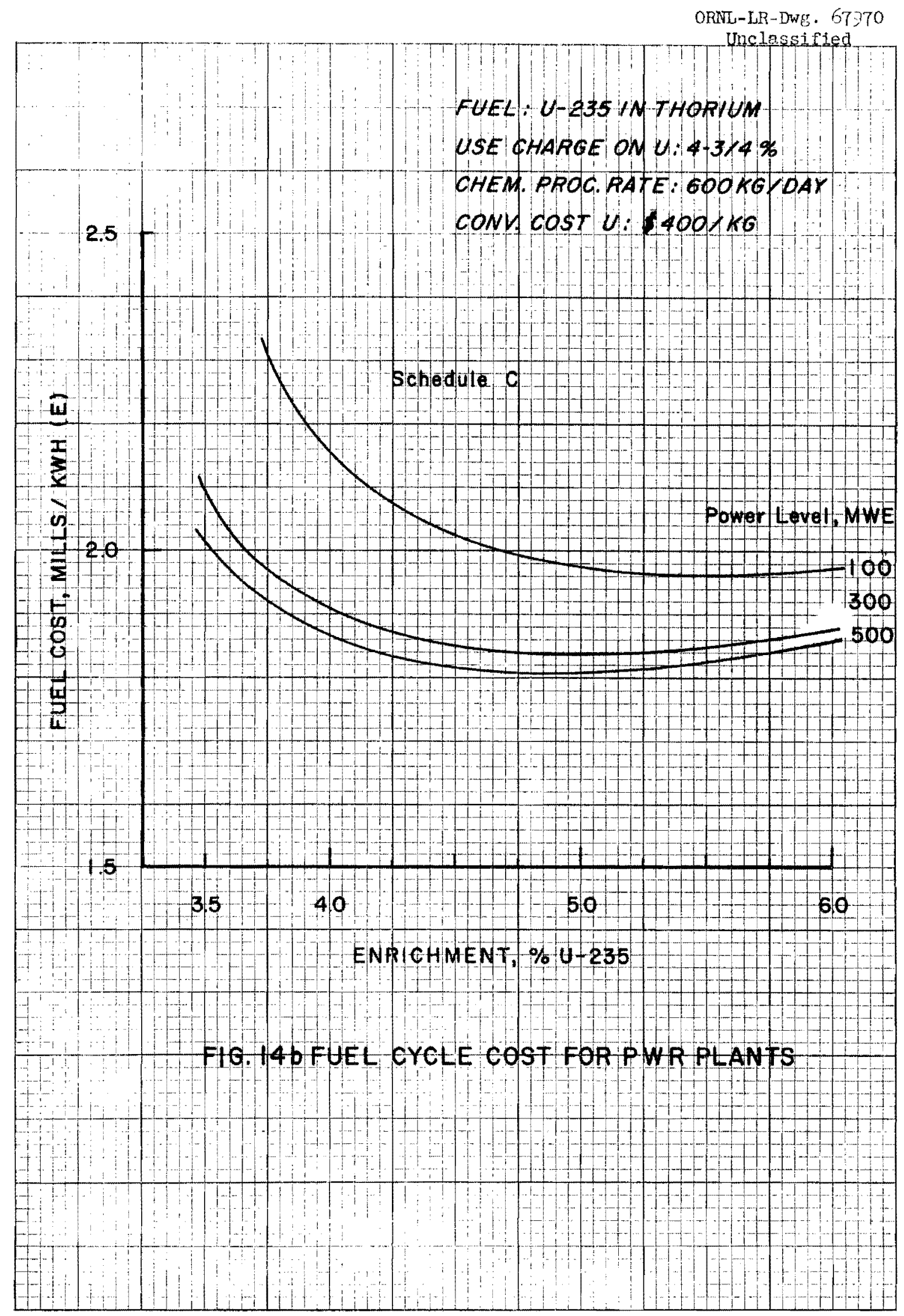


ORNL-LR-DWB . 67971

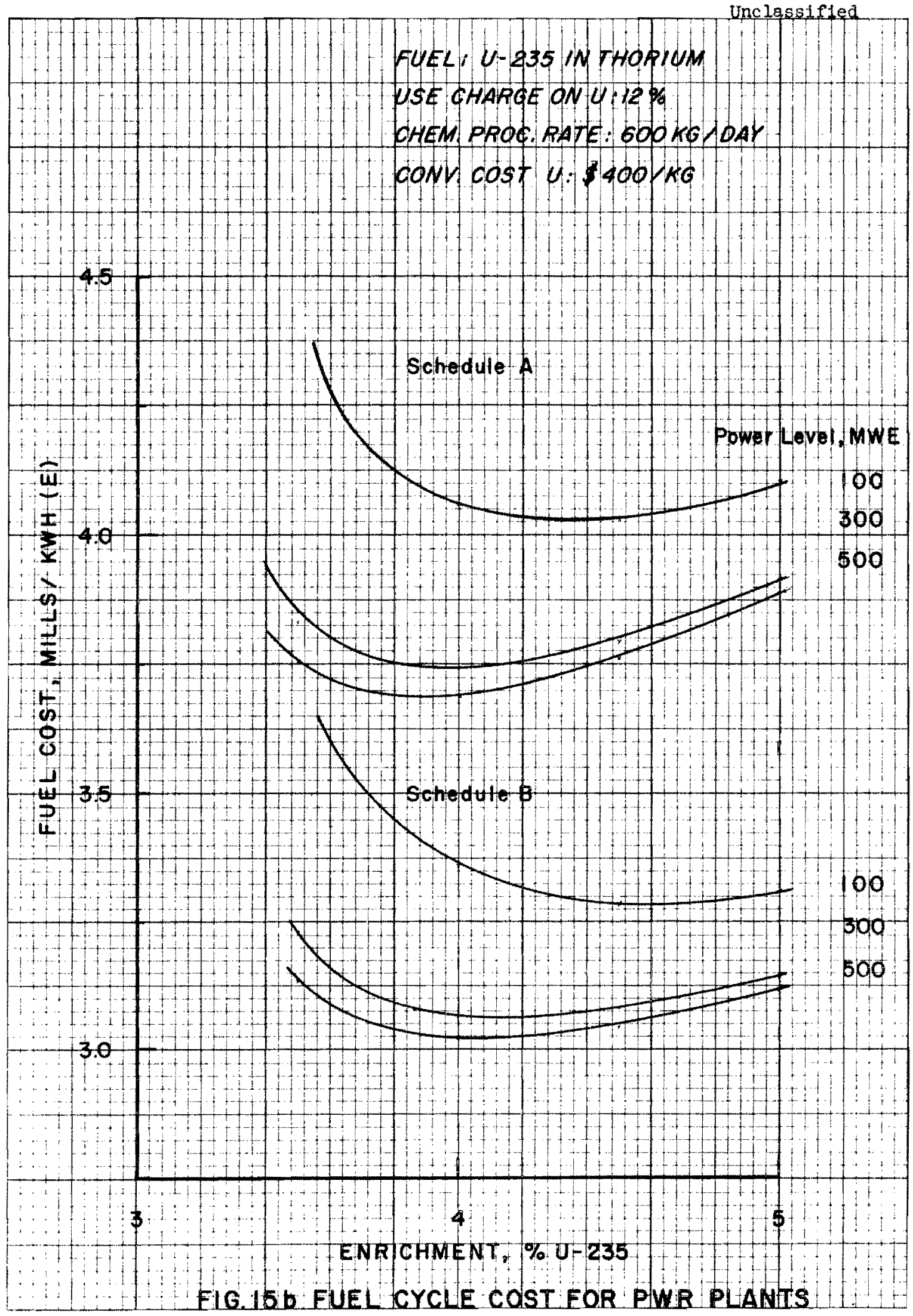




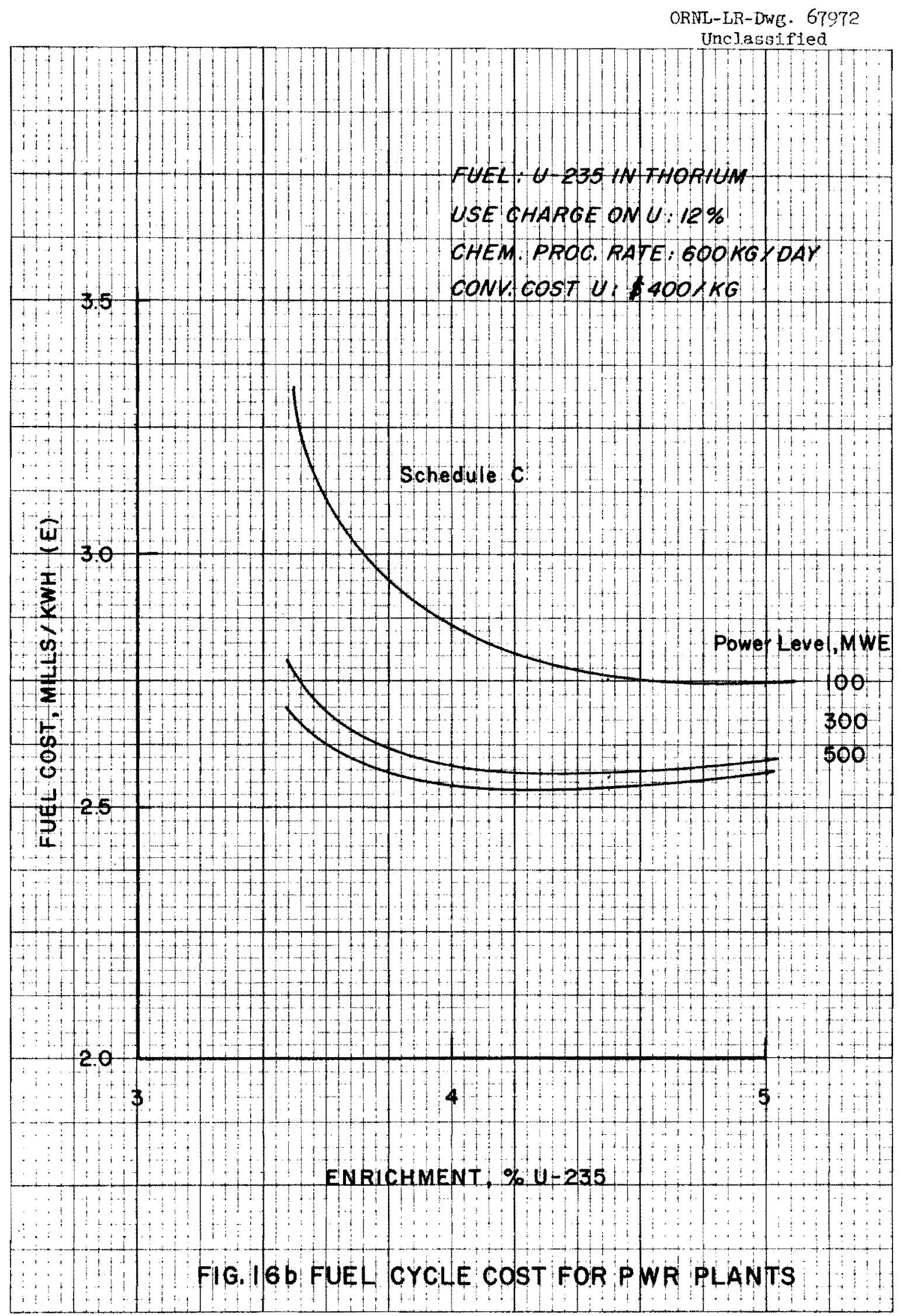


CR NL-LR-Dwg . 67973

unclaselfied

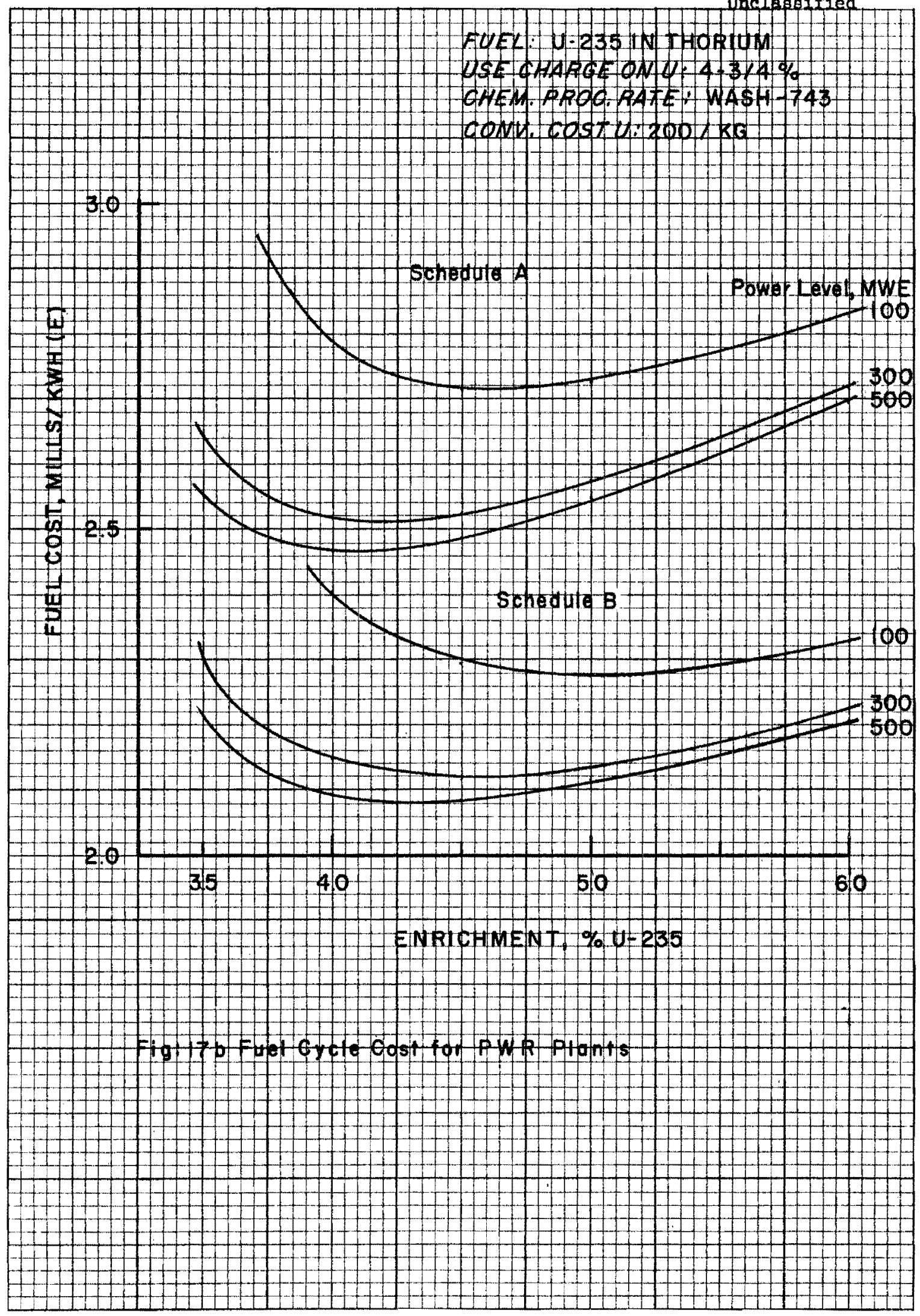


ORNL-LR-DWE . 67974

Unclassiffed

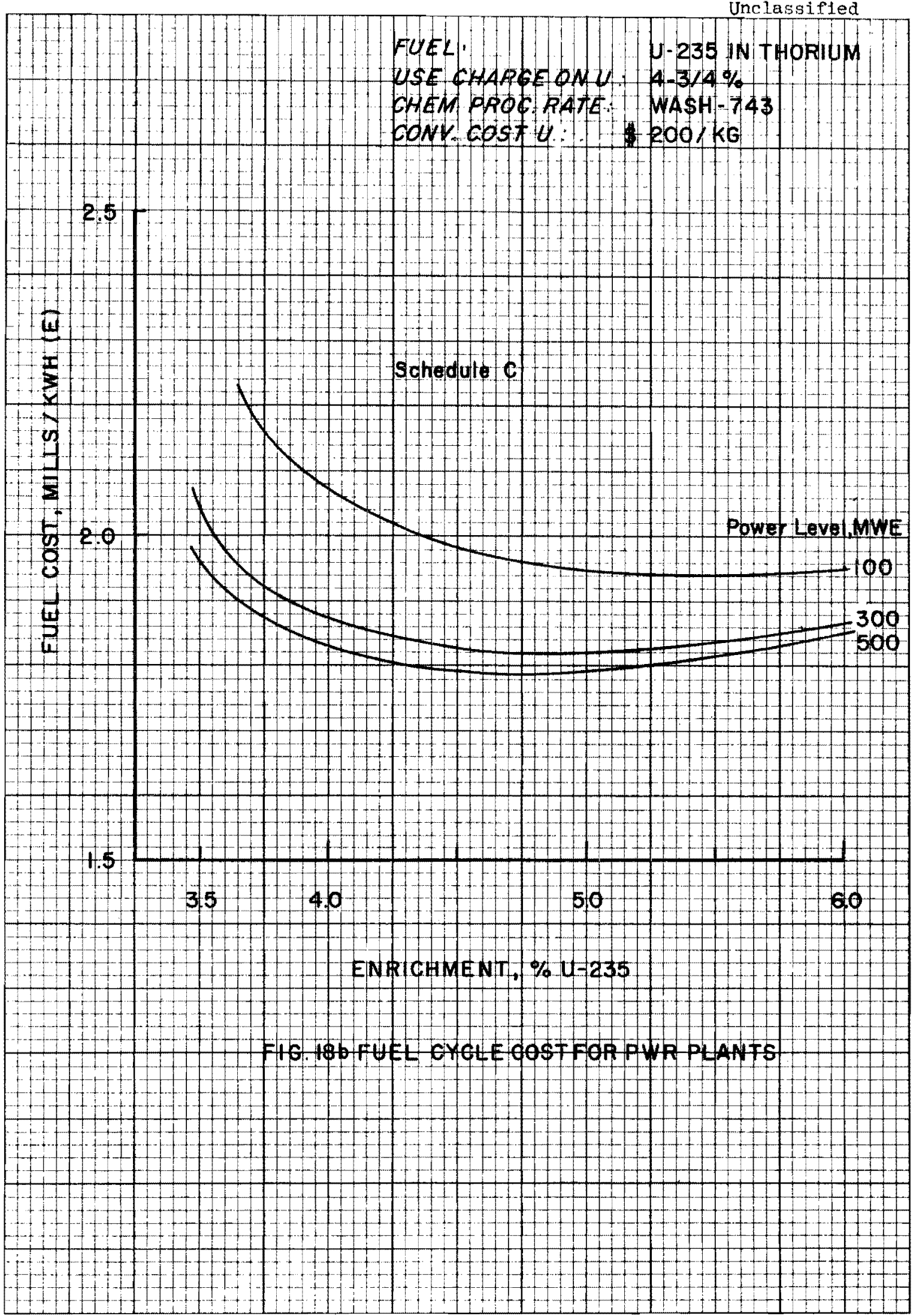


ORNL-LR-DWG . 67975

Unclessicied

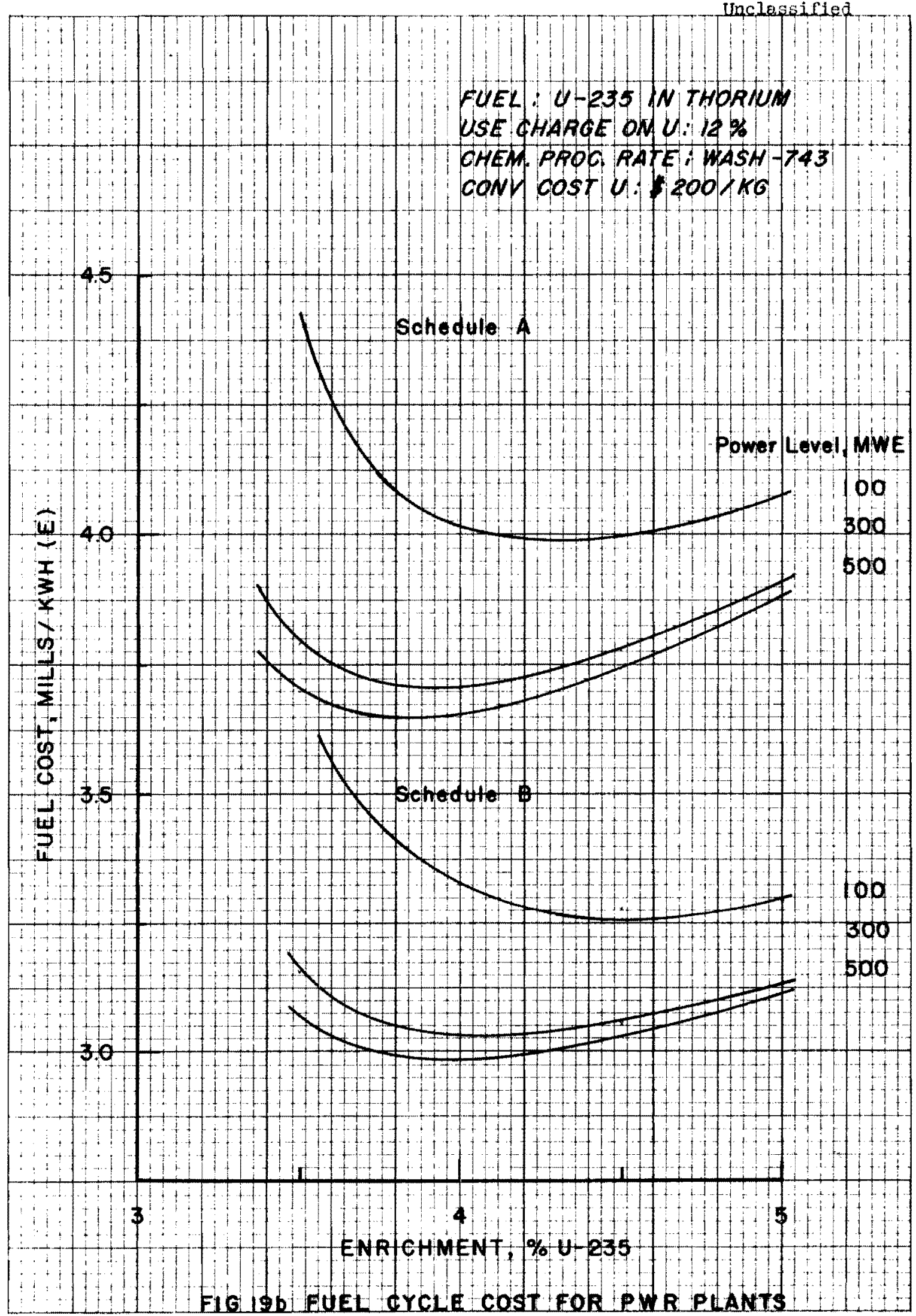




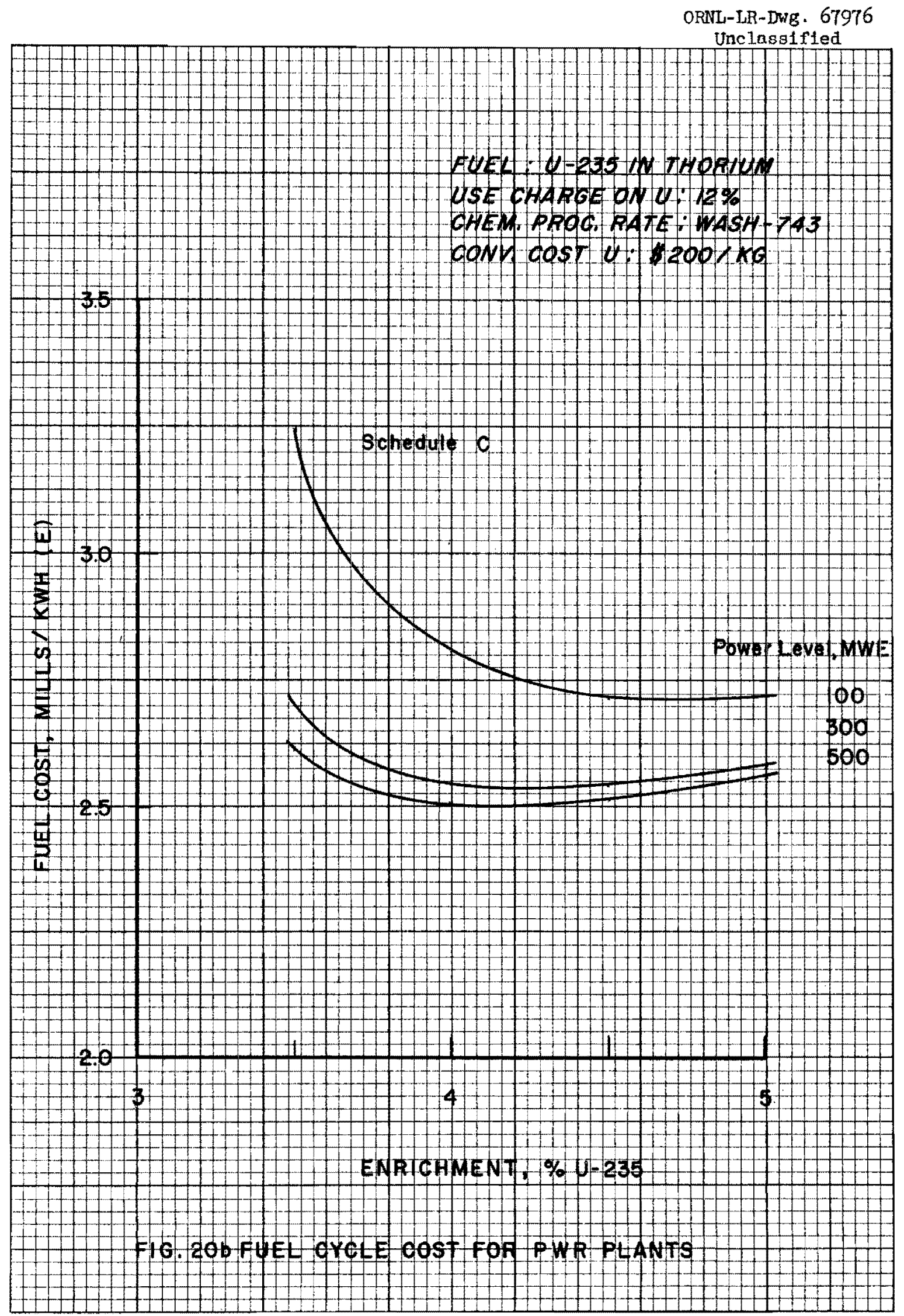


Distribution

1. I. G. Alexander
2. S. E. Beall
3. M. Bender
4-18. I. L. Bennett
19. A. L. Boch
20. R. B. Briggs
21. R. S. Carlsmith
22. W. L. Carter
23. R. D. Cheverton
24. H. C. Claiborne
25. J. G. Delene
26. T. B. Fowler
27. A. P. Fraas
28. E. H. Gift
29. D. R. Gilfillan
30-34. P. R. Kasten
35. T. W. Kerlin
36-40. J. A. Lane
41. R. N. Lyon
42. H. G. MacPherson
43. W. D. Manly
44. A. J. Miller
45. E. A. Nephew
46. C. W. Nestor
47. A. M. Perry
48. P. H. Pitkanen
49. C. A. Preskitt
50. M. W. Rosenthal

51. H, W. Savage

52. A. W. Savolainen

53. M. J. Skinner

54. 0. L. Smith

55. I. Spiewak

56. J. A. Swartout

57. M. Tobias

58. D. B. Trauger

59. Marina Tsagaris

60. R. Van Winkle

61. D, R. Vondy

62. A. M. Weinberg

63-64. Central Research Library

65-67. Y-12 Doc. Ref. Section

68-70. Laboratory Records Dept.

71. (IRD-RC)

\section{EXTERNAL}

72-86. Div. of Technical Information Extension

87. Research and Development Division, ORO

88-89. Reactor Division, ORO 


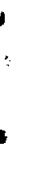

,

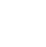

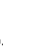

